Supporting Information

\title{
CGRtools: Python Library for Molecule, Reaction and Condensed Graph of Reaction Processing
}

Ramil I. Nugmanov ${ }^{\dagger}$, Ravil N. Mukhametgaleev ${ }^{\dagger}$, Tagir Akhmetshin $^{\dagger}$, Timur R. Gimadiev $^{\dagger}$, Valentina A. Afonina ${ }^{\dagger}$, Timur I. Madzhidov ${ }^{\dagger}, *$, Alexandre Varnek ${ }^{\#, *}$

${ }^{+}$Chemoinformatics and Molecular Modeling Laboratory, A.M. Butlerov Institute of Chemistry, Kazan Federal University. 18 Kremlyovskaya Str., 420008 Kazan, Russia

Timur.Madzhidov@kpfu.ru

\# Laboratory of Chemoinformatics, Université de Strasbourg. 4 rue Blaise Pascal, 67000 Strasbourg, France

varnek@unistra.fr 
Figure S1.

Mentioning of different programming languages in abstracts of 4 major chemoinformatics journals (JCIM, JCAMD, JCheminf, Mollnf) according to Web of Knowledge system. C/C++/C\# was not included due to technical reasons (selected papers does not correspond to search terms). For $\mathrm{R}$, search terms was "CRAN", "R language"; R2 was ignored - it returned only valid papers with real R mentioning.

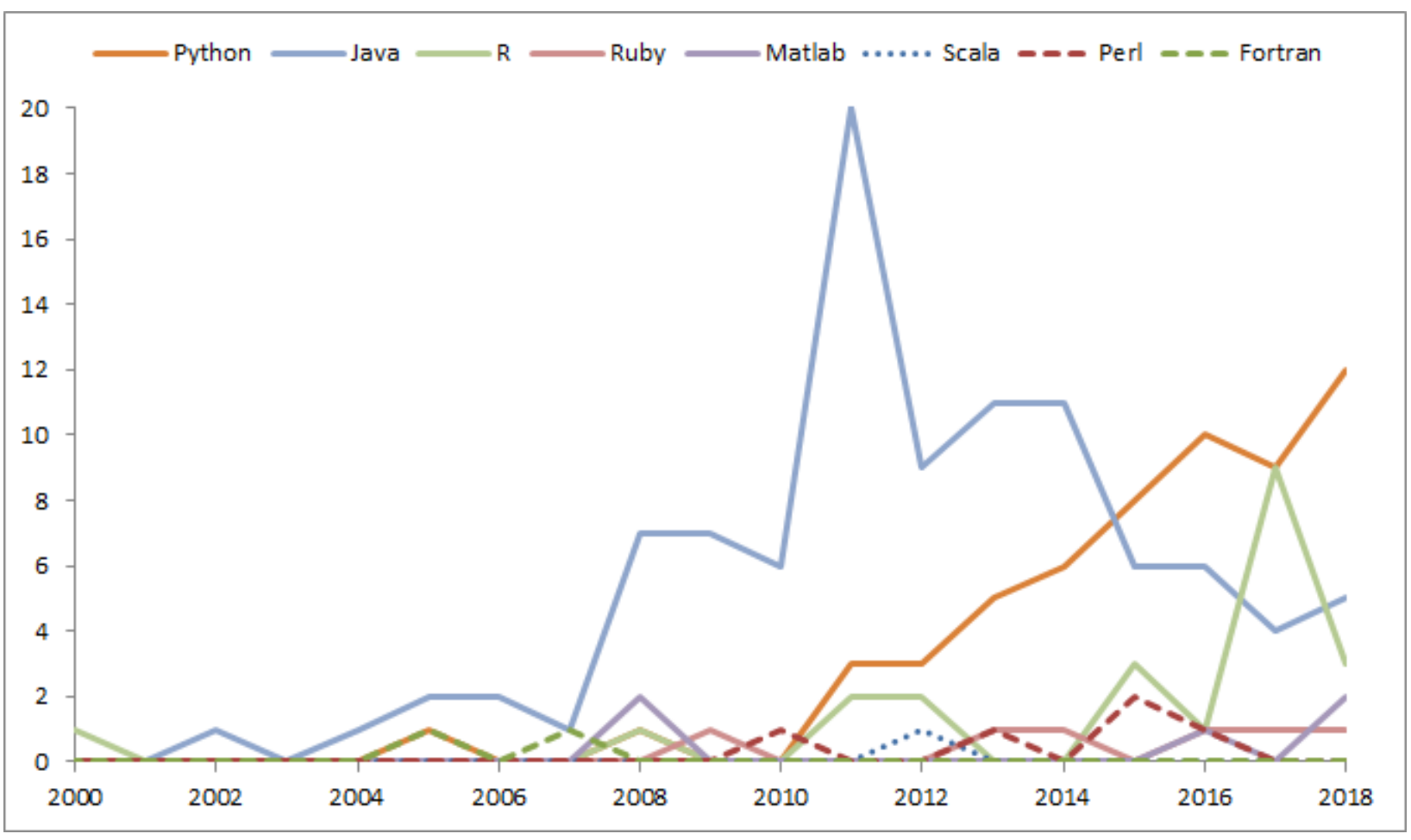




\section{Table S1. Comparison of CGRtools with other open source chemoinformatics toolkits.}

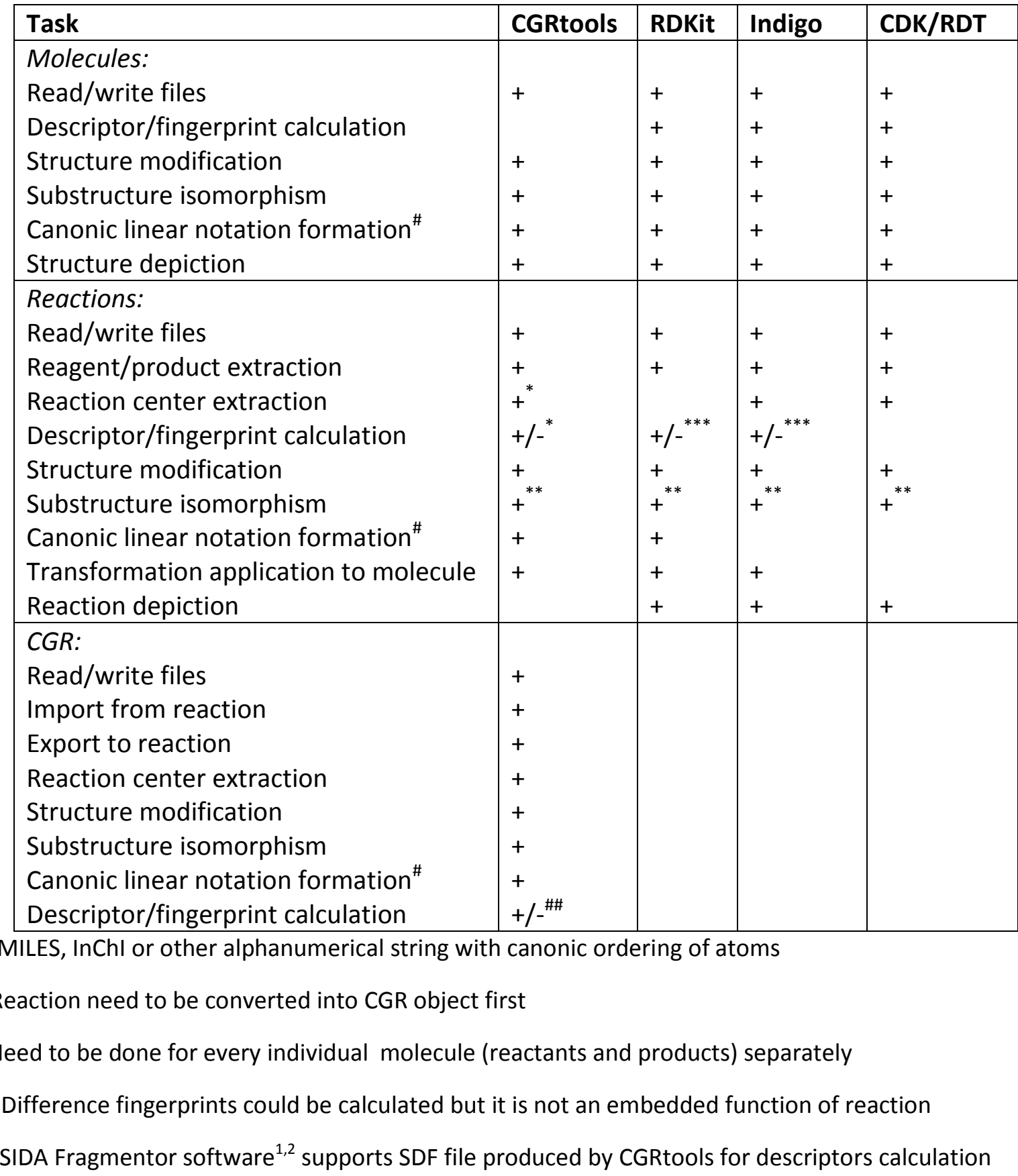


Testing have been performed in one thread using Intel(R) Xeon(R) CPU E5-2640 v4 @ 2.40GHz with 160 $\mathrm{Mb} / \mathrm{s}$ read limit hard drive. 1000 random reactions from USPTO reaction dataset ${ }^{3}$ was used. Average number of atoms in reactant and product part of reactions in the dataset is 56.

\begin{tabular}{|l|l|}
\hline Operation & $\begin{array}{l}\text { Average speed, } \\
\text { reaction per minute }\end{array}$ \\
\hline RDF file read & 24000 \\
\hline RDF file writing & 100000 \\
\hline $\begin{array}{l}\text { Enumeration of reagents and products and average number of atoms } \\
\text { in reaction calculation }\end{array}$ & 6000000 \\
\hline CGR generation from reaction & 20000 \\
\hline Reaction centers extraction from CGR & 430000 \\
\hline Reaction signature generation & 40000 \\
\hline Python 'set' data type generation based on reaction dataset & 30000 \\
\hline $\begin{array}{l}\text { Standardization procedure that includes groups standardization and } \\
\text { aromatization }\end{array}$ & 60000 \\
\hline $\begin{array}{l}\text { Substructure search of benzene ring in reaction CGRs (386 hits } \\
\text { retrieved) }\end{array}$ & 100000 \\
\hline $\begin{array}{l}\text { Generation of products using CGRtools Reactor based on template } \\
\text { transformation and dataset of } 1000 \text { molecules (386 new molecules } \\
\text { were generated) }\end{array}$ & 75000 \\
\hline
\end{tabular}




\section{Appendix A1. Comparison of some operations in CGRtools and RDKit}

Example of code with some comments is given.

\begin{tabular}{|c|c|c|}
\hline & CGRtools & RDKit \\
\hline SMILES files read & $\begin{array}{l}\text { \# Python generator object. Skips invalid data. } \\
\text { files.SMILESread('file') }\end{array}$ & $\begin{array}{l}\text { \# Not valid iterator. Returns None for invalid structures } \\
\text { Chem.SmilesMolSupplier('file') }\end{array}$ \\
\hline SMILES read & files.SMILESread (io.StringIO('CCO')) & Chem.MolFromSmiles ('CCO') \\
\hline SMIRKS read & files.SMILESread (io.StringIO ('C $(=0) 0>>C=0$ ') ) & AllChem. ReactionFromSmarts ( 'C $(=0) 0>>C=0$ ') \\
\hline SDF files read & $\begin{array}{l}\text { \# Same as for SMILES } \\
\text { files.SDFread('file') }\end{array}$ & Chem.SDMolSupplier('file') \\
\hline SDF files objects & $\begin{array}{l}\text { obj }=\text { open('file') } \\
\text { files.SDFread(obj) }\end{array}$ & $\begin{array}{l}\text { obj = open('file') } \\
\text { Chem. ForwardSDMolSupplier(obj) }\end{array}$ \\
\hline MOL file read & files.SDFread('file') & Chem.MolFromMolfile('file') \\
\hline RXN file read & files.RDFread('file') & AllChem.ReactionFromRxnFile('file') \\
\hline SDF files write & $\begin{array}{l}\text { w = files.SDFwrite('out_file') } \\
\text { w.write(molecule) } \\
\text { w. close() } \\
\text { \# Or using context } \\
\text { with SDFwrite('out_file') as w: } \\
\quad \text { w.write(molecule) }\end{array}$ & $\begin{array}{l}\mathrm{w}=\text { Chem.SDWriter('out_file') } \\
\mathrm{w} \cdot \text { write(molecule) }\end{array}$ \\
\hline
\end{tabular}




\begin{tabular}{|c|c|c|}
\hline MOL string creation & $\begin{array}{l}\text { \# files.SDFwrite with StringlO file emulation } \\
\text { with StringIO() as f, SDFwrite(f) as } \mathrm{s} \text { : } \\
\text { S.write (m) } \\
\text { ms = f.getvalue() [:-5] \# skip \$\$\$ }\end{array}$ & $\mathrm{ms}=$ Chem.MolToMolBlock $(\mathrm{m})$ \\
\hline $\begin{array}{l}\text { Number of atoms in } \\
\text { molecule }\end{array}$ & $\begin{array}{l}\text { len (molecule) } \\
\text { \# Other possibility } \\
\text { molecule.atoms_count }\end{array}$ & molecule. GetNumAtoms () \\
\hline $\begin{array}{l}\text { Number of bonds in } \\
\text { molecule }\end{array}$ & molecule.bonds_count & molecule. GetNumBonds () \\
\hline $\begin{array}{l}\text { Looping over atoms } \\
\text { and get atom } \\
\text { number }\end{array}$ & $\begin{array}{l}\text { for } \mathrm{n} \text {, atom in m.atoms (): } \\
\text { atom.number }\end{array}$ & $\begin{array}{l}\text { for atom in m.GetAtoms (): } \\
\text { atom. GetAtomicNum() }\end{array}$ \\
\hline $\begin{array}{l}\text { Looping over bonds } \\
\text { and get bond order } \\
\text { and incident atoms }\end{array}$ & $\begin{array}{l}\text { for first_atom, second_atom, bond in m.bonds(): } \\
\text { bond.order }\end{array}$ & $\begin{array}{l}\text { for bond in m. GetBonds (): } \\
\text { first_atom = bond. GetBeginAtom( ) } \\
\text { second_atom = bond. GetEndAtom( ) } \\
\text { bond. GetBondType () }\end{array}$ \\
\hline $\begin{array}{l}\text { Request individual } \\
\text { bonds or atoms }\end{array}$ & $\begin{array}{l}\text { \#element type for atom } 1 \\
\text { molecule.atom }(1) \cdot \text { element } \\
\text { \#bond order for bond between atoms } 1 \text { and } 2 \\
\text { molecule.bond }(1,2) \text {.order } \\
\text { \# other possibility } \\
\text { molecule [1] [2].order }\end{array}$ & $\begin{array}{l}\text { molecule.GetAtomWithIdx (1). GetSymbol () } \\
\text { molecule.GetBondBetweenAtoms }(1,2) \text {. GetBondType () }\end{array}$ \\
\hline $\begin{array}{l}\text { Select atom } \\
\text { neighbors }\end{array}$ & $\begin{array}{l}\text { \# Tuple of bond, atom pairs: } \\
\text { molecule.environment (1) }\end{array}$ & $\begin{array}{l}\text { atom }=\text { molecule.GetAtomWithIdx (1) } \\
\text { atom. GetNeighbors () }\end{array}$ \\
\hline
\end{tabular}




\begin{tabular}{|c|c|c|}
\hline & $\begin{array}{l}\text { \# Dictionary of neighbors idx as keys and bonds as values } \\
\text { molecule [1] } \\
\text { \# List, similar to RDKit GetNeighbors: } \\
\text { [molecule.atom (x) for } x \text { in molecule [1] ] }\end{array}$ & \\
\hline $\begin{array}{l}\text { Substructure } \\
\text { matching }\end{array}$ & $\begin{array}{l}\text { superstructure >= substructure } \\
\text { \# Mapping of substructure atoms to superstructure: dictionary with } \\
\text { indexes of substructure atoms as keys and superstructure atoms } \\
\text { indexes as values } \\
\text { match_dict = } \\
\text { substructure.get_substructure_mapping (superstru } \\
\text { cture) }\end{array}$ & $\begin{array}{l}\text { superstructure.HasSubstructMatch (substructure) } \\
\text { \# Mapping of substructure atoms to superstructure: tuple with atom } \\
\text { numbers in superstructure in order of atom numbers in substructure } \\
\text { match = super_m.GetSubstructMatch (sub_m) }\end{array}$ \\
\hline $\begin{array}{l}\text { Duplicates filtering } \\
\text { Check if molecule } \\
\text { exist in a given set }\end{array}$ & $\begin{array}{l}\text { \# Remove duplicated molecules } \\
\text { unique }=\text { set (molecules) } \\
\text { \# Molecules should be a represented as set: } \\
\text { molecule in unique }\end{array}$ & $\begin{array}{l}\text { unique }=\{\} \\
\text { for molecule in molecules: } \\
\quad \text { unique [Chem. MoltoSmiles (molecule) ] = molecule } \\
\text { \# Direct checking impossible. You can create dict keyed by smiles. } \\
\text { Chem.MolToSmiles (molecule) in unique }\end{array}$ \\
\hline $\begin{array}{l}\text { Aromatizing and } \\
\text { Valence checking }\end{array}$ & $\begin{array}{l}\text { molecule.aromatize() } \\
\text { molecule.check_valence() } \\
\text { \# Group transformation }\end{array}$ & $\begin{array}{l}\text { \# Aromatizing and valence checking } \\
\text { Chem.SanitizeMol (molecule) }\end{array}$ \\
\hline
\end{tabular}




\begin{tabular}{|c|c|c|}
\hline & molecule.standardize() & $\begin{array}{l}\text { \# Group transformation not supported directly. You can use SMARTS } \\
\text { \# rules as shown in part "Application of transformation rule to a } \\
\text { \# molecule and generation of product and reaction" }\end{array}$ \\
\hline $\begin{array}{l}\text { Application of } \\
\text { transformation rule } \\
\text { to a molecule and } \\
\text { generation of } \\
\text { product and reaction }\end{array}$ & 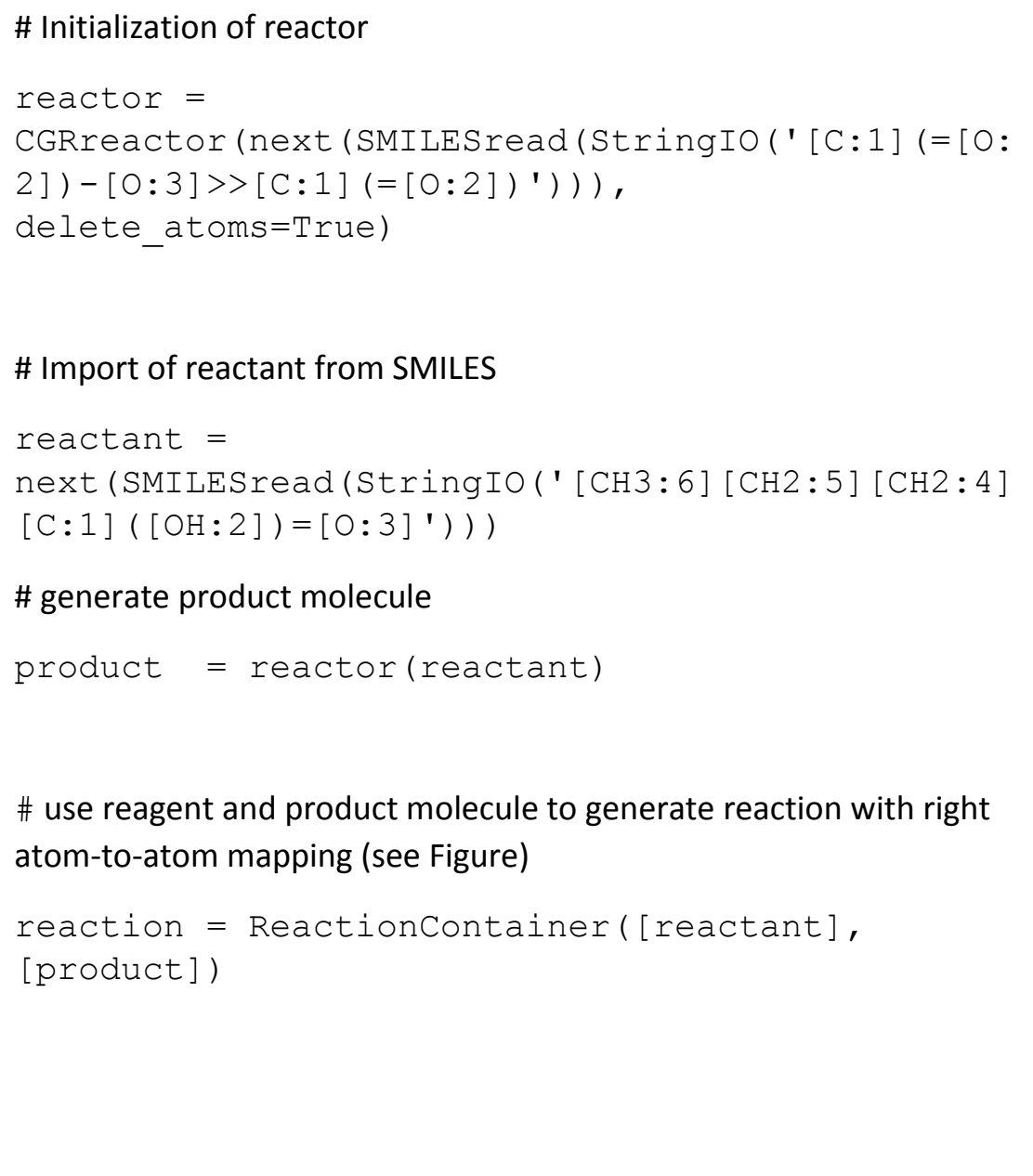 & 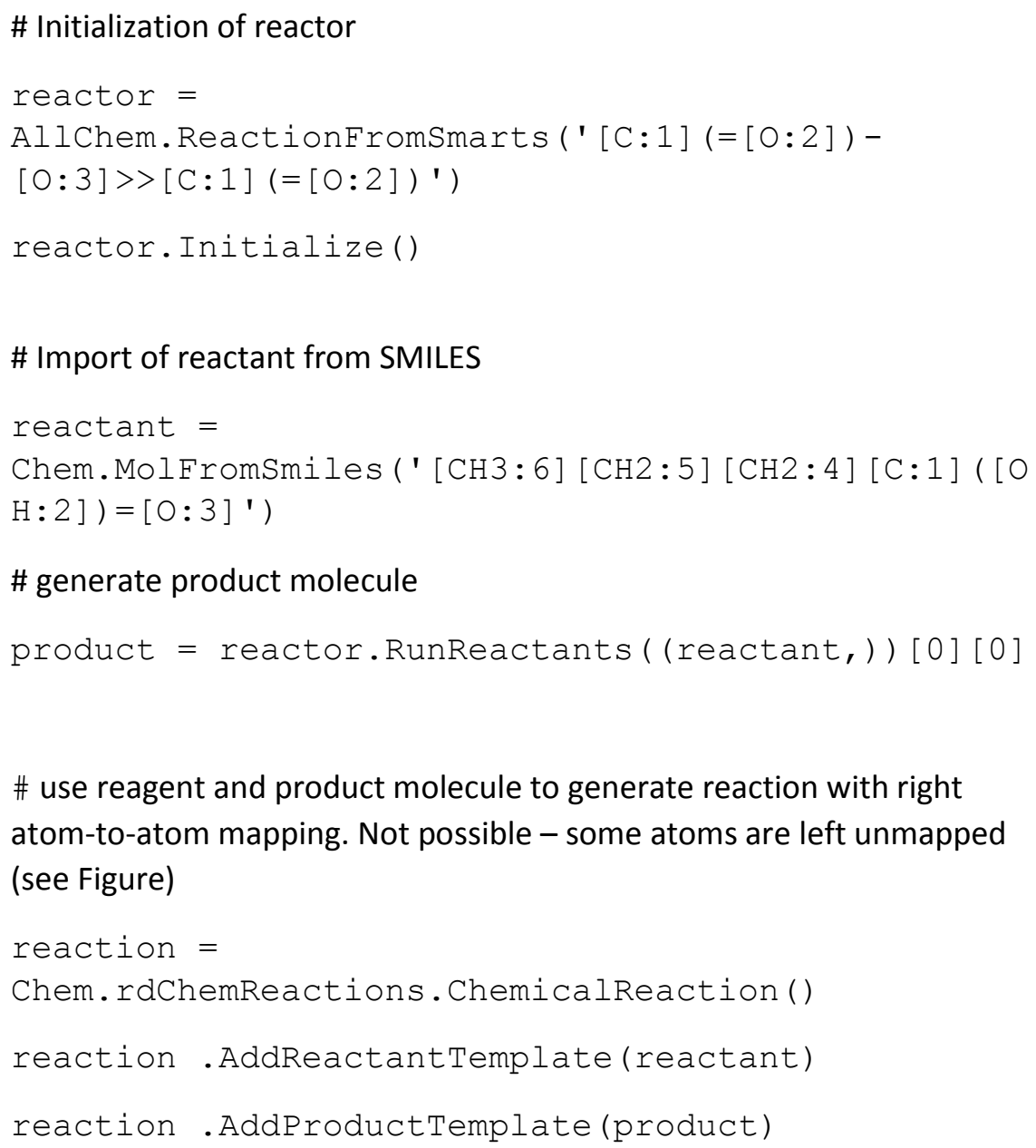 \\
\hline
\end{tabular}




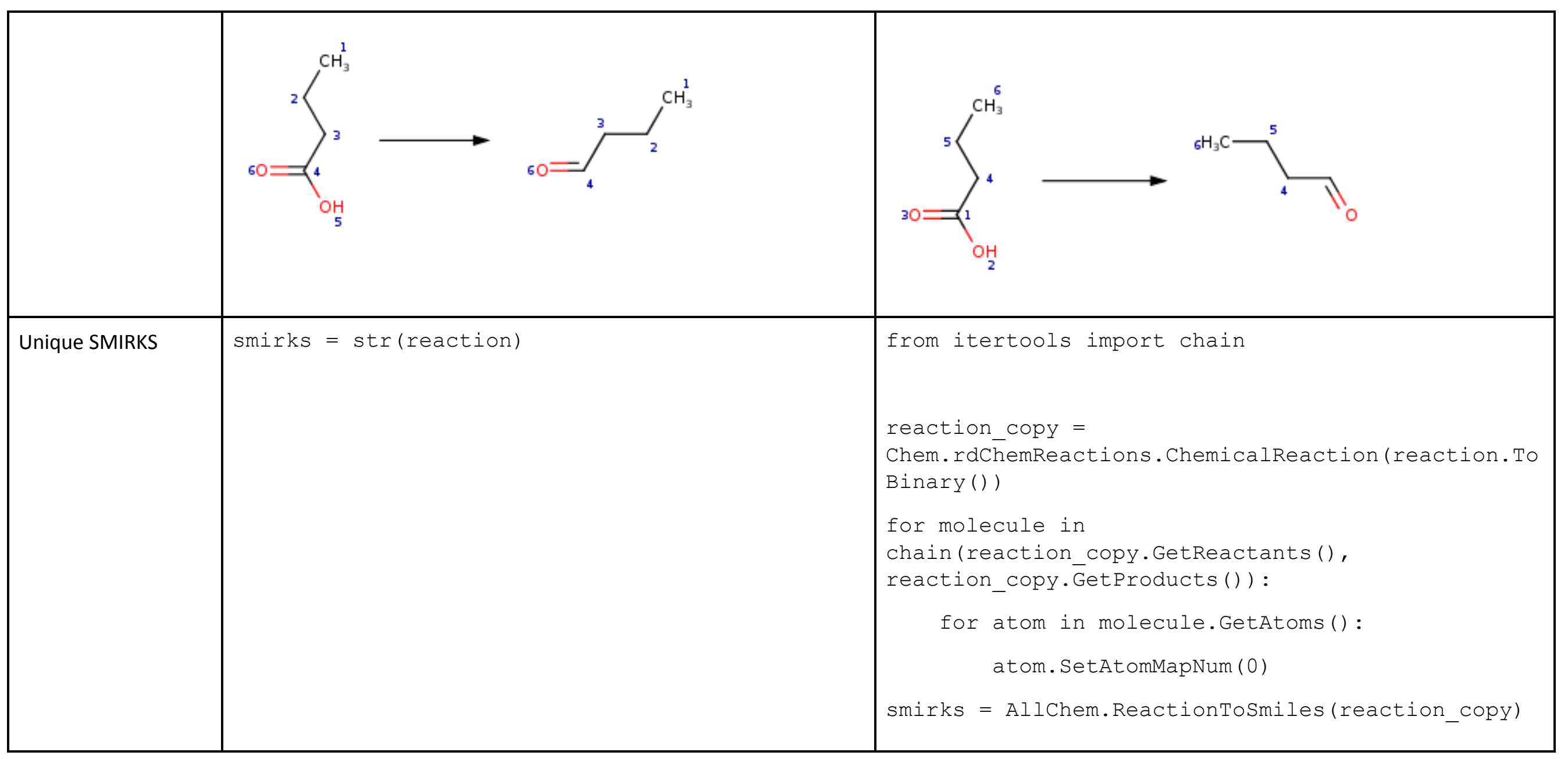




\section{Appendix A2. Molecule, reaction and CGR signature generation.}

Signatures are unique identifier for molecule, reaction or CGR, that is used for generation hashes keys. The hash key makes any chemical objects hashable. In current implementation they are sensitive to atom and bond types, i.e. aromaticity, tautomeric state, molecules standardization.

As signatures SMILES like alphanumerical strings are generated based on OpenSMILES ${ }^{4}$ rules using canonic atom numbering. Canonic ordering algorithm used for atom numbering is described below. Then we describe rules for text strings for objects of different type.

\section{Canonic ordering algorithm}

Algorithm is based on Morgan canonicalization scheme ${ }^{5}$, CANGEN algorithm idea on application of ranks instead of extended connectivity ${ }^{6}$ and property of unique factorization of any integer into prime numbers used by Gasteiger and Ihlenfeldt in their molecular hash generation algorithm ${ }^{7}$.

The molecule is treated as a graph with labelled nodes (atoms) and edges (bonds). Each atom is assigned an integer based on element symbol, formal charge, isotope and multiplicity. Thus, atom type is represented by 21 bit code:

- 7 bits stand for atom number $(2 * * 7-1==127$, currently 118 elements are presented in Periodic Table)

- $\quad 9$ bits are used for isotope (511 possibilities, highest known isotope is 300)

- 3 bits stand for formal charge. Charges range from -3 to +3 rescaled to range 0-6

- 2 bits are used for multiplicity.

Bit number is rounded to 32 bit integer number which is used.

Each bond from atom to their neighbors also encoded as integer representing bond order: 1, 2, 3, 4 (aromatic bond). Bond orders incident to atom are sorted and saved as tuple. Combination of atom integer code and sorted tuple of bond orders used as keys of atoms. In next step sorted atoms keys are replaced with prime numbers. Equal keys has equal prime numbers.

In second stage for each atom square of its prime number is multiplied by neighboring atoms prime numbers. New values are sorted and again replaced by prime numbers.

This procedure is repeated until all atoms are assigned unique prime numbers or number of unique atomic values does not change in 3 subsequent iterations. For very rare cases if oscillation is started, number of iterations is limited to $4 *$ number_of_atoms_in_molecule.

General workflow of canonicalization is the following:

1: For each atom get its type (element type, isotope, charge, multiplicity are considered) encoded by integer. Sort incident bond orders and make tuple of them. Represent every atom as tuple, consisting from atom type coding integer and sorted bond order tuple: (atom, (bond1, bond2))

2: Sort list of atom-bonds tuples. Calculate ranks. The same tuples have the same rank.

3: Replace ranks of atom-bonds tuples by prime numbers from lookup table starting from 2 in ascending order. The same prime numbers are assigned to atom-bond tuples having the same rank. 
4: For each atom multiply square of its prime number with prime numbers of neighboring atoms.

5: Sort obtained numbers for atoms. Replace them with prime numbers beginning with first (2) in ascending order as in step 3 . The same numbers are replaced by the same prime numbers.

6: Check if every atom has unique prime number. If yes - finish algorithm.

7: Check if number of unique prime numbers assigned does not change last 3 iterations. If yes - finish algorithm.

8: If number of iterations exceeds $4 *$ number_of_atoms - finish algorithm.

9: Start new iteration, go to step 4.

\section{Signature generation}

For molecules based on given canonic numbering, algorithm generates SMILES strings using OpenSMILES ${ }^{4}$ rules. Resulting alphanumerical string is readable by most of programs supporting OpenSMILES.

For reactions, canonic numbering is done by joining all molecules of reagent/products/reactants (separately) into one graph. Then, SMILES-like strings for all molecules are built using observed canonic numbering. Molecules are ordered according to atoms' ranks. In such a way signatures for reagent, product and reactant parts are independent on initial order of molecules, presence or absence of disconnected graph components. Final signature is created uniting this three: reactants' signature>reagents' signature>products' signature. In such a way a reaction signatures represent valid unique reaction SMILES.

For CGR canonic numbering is performed using algorithm similar to that for the molecules. Since in CGR some atom and bond labels reflect changes of atomic properties (charge, multiplicity) or bond order in reaction, two SMILES-like strings are created in order to enumerate atoms, Figure 1. In the first string, atoms and bonds are shown as in reagent part and in the second string - as in product part, for absent bond dot symbol is used. Final text string is produced by concatenation of two SMILES-like strings separated by > symbol. Since dot symbols application violate OpenSMILES rules, the CGR signatures are not readable by popular software tools, but are still human readable. 
Version 1.1

\author{
Laboratory of Chemoinformatics, University of Strasbourg, France
}

Laboratory of Chemoinformatics and Molecular Modeling,

Kazan Federal University, Russia

This report aims at comparing the Condensed Graph of Reaction (CGR) system developed since 2005 in Strasbourg (Unistra) and it current implementation in the laboratory of Chemoinformatics of the Kazan Federal University (KFU).

\title{
Condensed Graph of Reaction
}

A CGR is a supergraph, which subgraphs include the reactive and products of a Chemical reaction (Figure 1). New chemical bond types, the dynamical bonds, are introduced to code the creation, deletion and bond type modifications resulting from the reaction ${ }^{2,8}$.

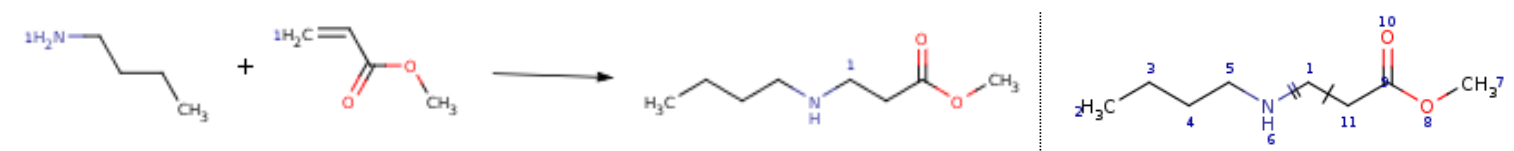

Figure 1: Illustration of a CGR. On the left, the classical representation of a reaction. On the right the corresponding CGR.

Historical overview of CGR encoding

Technically, the CGR is encoded into an ASCII text file following the MDL/SDF format ${ }^{9,10}$.

The legacy implementation of the Unistra/CGR is based on a free interpretation of the bond block of the SDF format. Initially a small set of transformations was encoded as special bond types ${ }^{2,8}$. The problem was that the CGR could be edited only by dedicated software developed in house.

To solve the problem, the decision was taken in 2010 to change the format. The dynamic bonds were coded as a combination of a normal bond type and additional information encoded in another column of the bond block. The encoding system is reminded in the following tables (Table 1, Table 2). This system was historically included by MDL in order to manage queries for chemical bond transformations in reaction databases. The advantage is that most common chemical editors can read and edit a CGR file and the rendering by third party software is rather close to the correct interpretation of the dynamic bonds into a CGR. The table used to encode the transformations is given below. The current system works and can be further extended to include new conventions. The limitation is that the CGR/Unistra proposition is an unofficial interpretation of the MDL/SDF format. Addition of new conventions for dynamic atoms, (dynamic) stereochemistry of bonds and atoms will lead to new interpretations of existing fields and finally it would not be useful or even possible. 
Table 1: List of bond encoding using the legacy MDL/SDF format.

\begin{tabular}{|l|l|l|l|}
\hline Bond Type & Symbol & SDF bond column 3 & SDF column 7 \\
\hline Simple & - & 1 & 0 or 2 \\
\hline Double & $=$ & 2 & 0 or 2 \\
\hline Triple & + & 3 & 0 or 2 \\
\hline Aromatic & $*$ & 4 & 0 or 2 \\
\hline Single or Double & $\cdot$ & 5 & 0 or 2 \\
\hline Single or Aromatic & $:$ & 6 & 0 or 2 \\
\hline Double or Aromatic & $"$ & 7 & 0 or 2 \\
\hline Any bond type & $?$ & 8 & 0 or 2 \\
\hline Special bond type & - & 9 & 0 or 2 \\
\hline Single bond in cycle & $\cdot$ & 50 & 0 or 2 \\
\hline Double bond in cycle & $:$ & 60 & 0 or 2 \\
\hline Triple bond in cycle & $\#$ & 70 & 0 or 2 \\
\hline Hydrogen bonds & $\sim$ & 80 & 0 or 2 \\
\hline Unknown bond & YY & & \\
\hline
\end{tabular}

Table 2: List of dynamical bonds encoded using the legacy MDL/SDF format for Unistra/CGR

\begin{tabular}{|l|l|l|l|}
\hline Bond Type & Symbol & SDF bond column 3 & SDF column 7 \\
\hline Single bond creation & 81 & 1 & 8 \\
\hline Double bond creation & 82 & 2 & 4 \\
\hline Triple bond creation & 83 & 3 & 12 \\
\hline Aromatic bond creation & 84 & 4 & 1 \\
\hline Single bond cut & 18 & 1 & -1 \\
\hline Double bond cut & 28 & 2 & -1 \\
\hline Triple bond cut & 38 & 3 & -1 \\
\hline Aromatic bond cut & 48 & 4 & -1 \\
\hline Single bond to double bond & 12 & 2 & 8 \\
\hline Single bond to triple bond & 13 & 3 & 8 \\
\hline Single bond to aromatic bond & 24 & 4 & 8 \\
\hline Double bond to single bond & 21 & 1 & 4 \\
\hline
\end{tabular}




\begin{tabular}{|l|l|l|l|}
\hline Double bond to triple bond & 23 & 3 & 4 \\
\hline Double bond to aromatic bond & 24 & 4 & 4 \\
\hline Triple bond to single bond & 31 & 1 & 12 \\
\hline Triple bond to double bond & 32 & 2 & 12 \\
\hline Triple bond to aromatic bond & 34 & 4 & 12 \\
\hline Aromatic bond to single bond & 41 & 1 & 1 \\
\hline Aromatic bond to double bond & 42 & 2 & 1 \\
\hline Aromatic bond to triple bond & 43 & 3 & 1 \\
\hline
\end{tabular}

Attempts to model reaction by means of CGR was faced with the problems that reaction can have not only bond order change but also change of atom/bond stereochemistry and other atom properties. That's why conception of dynamic bonds was broadened to atoms and concept of dynamic atoms was proposed. Since conceptually this can be source for many new "dynamicities" more general approach for CGR representation in MDL/SDF was proposed in KFU.

For CGR/KFU, the decision was to keep the MDL/SDF definition untouched and use the Atom List Block. More precisely, it uses the Sgroup Type format, described in the MDL/CTFile document. The advantage is that any group of atom can receive an arbitrary label, and it is therefore very flexible to implement any coding convention. In future this approach can also be used for any arbitrary atom/bond property, for example, CGR query representation (how many hydrogen should be this atom connected to, degree of edge and so on).

This solution is compatible with a standard MDL/SDF format. The drawbacks are that the rendering of third party molecular editor are not very readable, that the edition of the dynamical bond requires to the editor to open an edition window instead of a list of choice (thus it is more open to human mistakes) and annotation of a bond annotation must be removed by a specific operation compared to the bond edition into an editor. The last drawback is that this SDF format contains more lines: each dynamic bond requires 3 additional lines per molecule to be coded.

\section{CGR Representation Specification}

The first step is to define a standard of the content of a CGR. This is a list of the information that must be encoded into a CGR.

Standard CGR information to encode is dynamic atom or dynamic bond specification.

Dynamic bond is a change in bond property that takes place during reaction transformation: change in bond order, bond stereochemistry.

Dynamic atom is a change in formal atom property that takes place in reaction: change of formal charge, stereochemistry, radical (valence state). 
Each CGR property is encoded in 3 fields. In the first field it is shown which atom or bond CGR property corresponds to. Precisely first field encode atomic array that can contain 1 entry (for dynamic atom) or 2 entries (for dynamic bonds). The second line contains title of property. The third line contains information needed for visualization of CGR. Forth line contains value of CGR property.

The fields look in general:

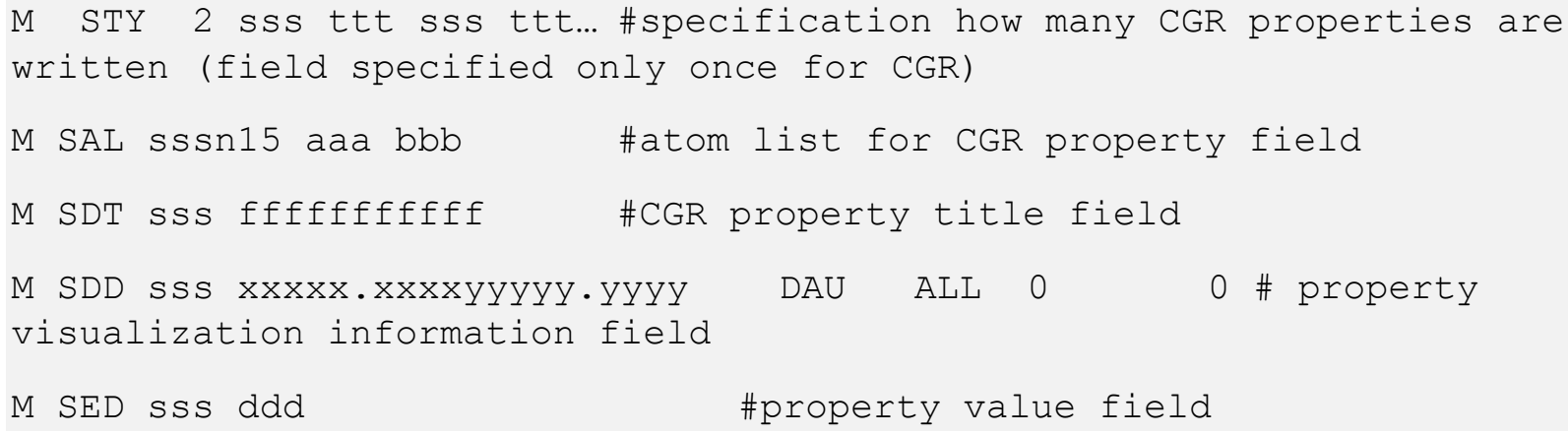

The last 4 lines repeates as much properties are need to be encoded.

Specification:

sss - ID of property (integer, from 1 to 999)

$t t t$ - Sgroup type (for CGR properties should be 'DAT' that means 'data Sgroup')

$n 15$ - number of atoms in the list (1 - for dynamic atom, 2 - for dynamic bond)

$a a a, b b b$ - atom numbers (according to atom block in normal $\mathrm{MOL}$ file)

ffffffffff-CGR property title

$x x x x x . x x x x, y y y y y . y y y y-x$ and $y$ coordinates for CGR property value position on renderer screen

$d d d$ - CGR property value (we propose to use 3-symbol notation)

DAU ALL $0 \quad 0$ - specific information from MDL/CTfiles. DAU means 'detached display, absolute position coordinates, display units', ALL - display all characters. Other characters are unused.

In M STY line up to more 69 symbols can be used. If it is not enough, enumeration of CGR properties should be followed on next line until all properties will be shown.

\section{Dynamic bonds context}

In the bond block, dynamic bonds are specified as 8 (any bond).

\section{CGR PROPERTY VALUES}

Acceptable values are shown in table 1. 
For dynamic bond types 3-symbol notations is used: " $\mathrm{B}>\mathrm{A}$ "

B - bond type symbol before reaction

A - bond type symbol after reaction

Table 1: Definition for bond types

\begin{tabular}{|c|c|}
\hline Bond type & Symbol \\
\hline no bond & 0 \\
\hline single & 1 \\
\hline double & 2 \\
\hline triple & 3 \\
\hline aromatic & 4 \\
\hline Special & 9 \\
\hline
\end{tabular}

Table 2: Table of possible values for dynamic bond type specification. "Before" shows bond order in left hand side of reaction equation, "after" is bond order in the right hand side of reaction equation.

\begin{tabular}{|l|l|l|l|l|l|l|}
\hline Before After & $\begin{array}{l}\text { No } \\
\text { bond }\end{array}$ & Single & Double & Triple & Aromatic & Special \\
\hline No bond & NA & $0>1$ & $0>2$ & $0>3$ & $0>4$ & $0>9$ \\
\hline Single & $1>0$ & NA & $1>2$ & $1>3$ & $1>4$ & $1>9$ \\
\hline Double & $2>0$ & $2>1$ & NA & $2>3$ & $2>4$ & $2>9$ \\
\hline Triple & $3>0$ & $3>1$ & $3>2$ & NA & $3>4$ & $3>9$ \\
\hline Aromatic & $4>0$ & $4>1$ & $4>2$ & $4>3$ & NA & $4>9$ \\
\hline Special & $9>0$ & $9>1$ & $9>2$ & $9>3$ & $9>4$ & NA \\
\hline
\end{tabular}

\section{Example:}

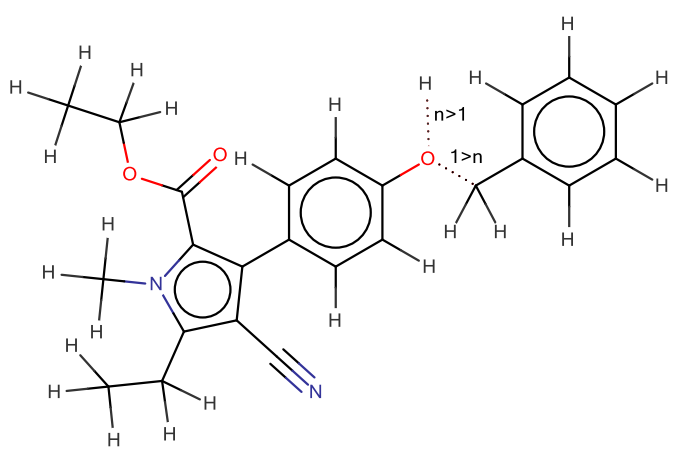

(made in Marvin Sketch, atom-to-atom mapping is hidden for clarity) 


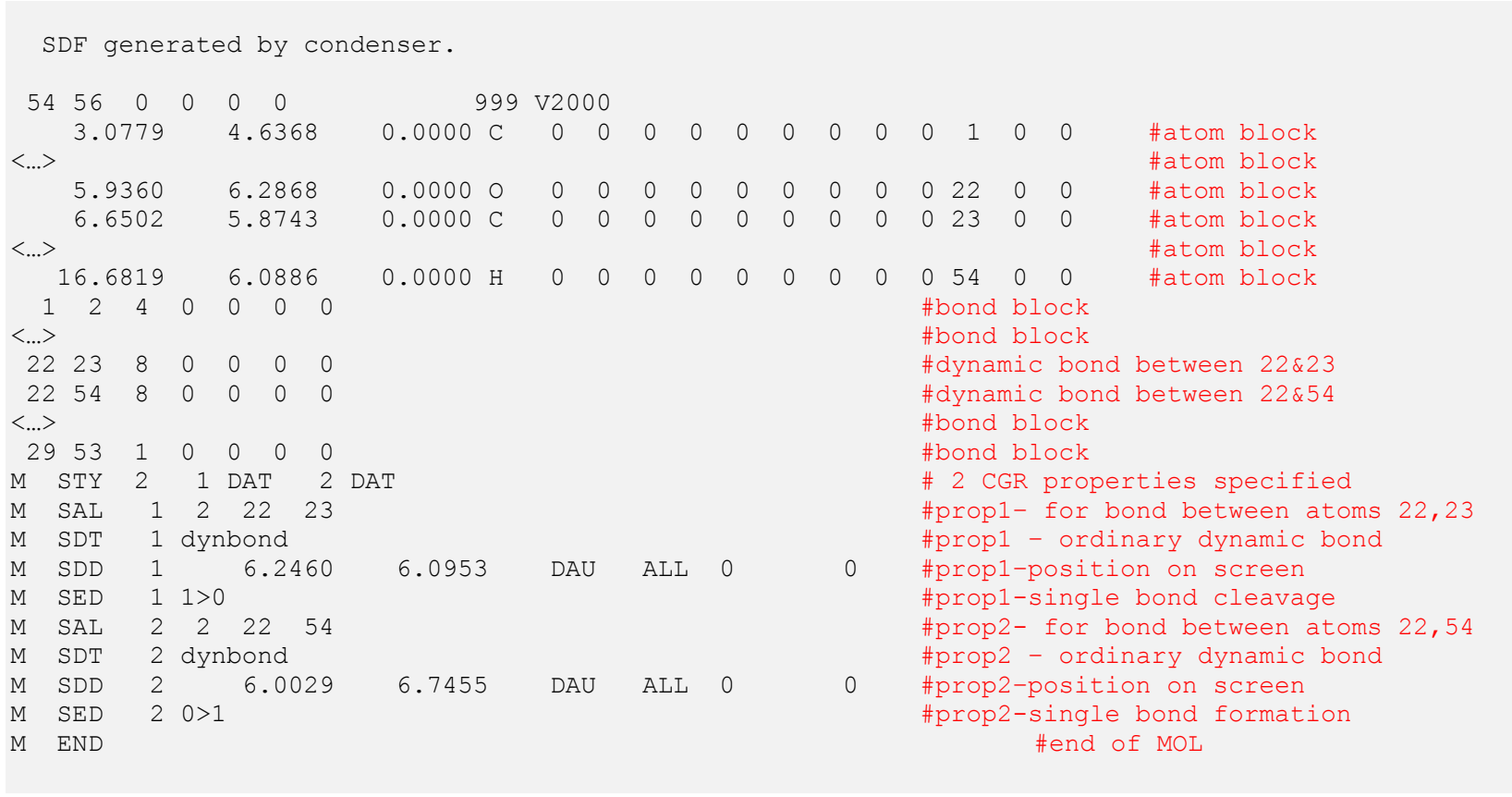

\section{Dynamic atoms context}

\section{Atomic valence property changes}

The CGR must be able to record changes in oxidation numbers or electron count: the change of a formal charge or the appearence or deletion of radical species. This property is atom based and therefore is best encoded using as an atomic property.

Theoretically, three types of change in atomic valence state can be: formal charge change, radical change, hydrogen count change (it is excluded since redundant with previous two).

For simplicity the following procedure for valence state specification is proposed:

CGR PROPERTY NAME "dynatom".

CGR PROPERTY DISPLAY

Valence state of atom in MOL file (in atom block) is specified as in reagent side.

CGR PROPERTY VALUE:

Possible values are formed in the following way:

$\mathrm{S} \pm \mathrm{V}$

S-symbol for property:

$\mathrm{c}$ - change in formal charge of atom

$r$-change in radical state on atom

\pm - sign of the change

$V$ - value of the change. 
Table 3: Table of most frequent dynamic atom charge.

\begin{tabular}{|l|l|l|l|}
\hline Value & Initial charge & Final charge & Notes \\
\hline$c+1$ & 0 & +1 & \\
\hline$c+1$ & -1 & 0 & \\
\hline$c+1$ & +1 & +2 & For metals mostly \\
\hline$c+1$ & -2 & -1 & For metals mostly \\
\hline$c-1$ & 0 & -1 & \\
\hline$c-1$ & +1 & 0 & For metals mostly \\
\hline$c-1$ & +2 & +1 & For metals mostly \\
\hline$c+2$ & 0 & +2 & For metals mostly \\
\hline & +1 & +3 & \\
\hline
\end{tabular}

Table 4: Table of most frequent dynamic atom radical states.

\begin{tabular}{|c|c|c|c|c|}
\hline Value & $\begin{array}{l}\text { Initial number } \\
\text { of electrons in } \\
\text { open shell }\end{array}$ & $\begin{array}{l}\text { Final number } \\
\text { of electrons in } \\
\text { open shell }\end{array}$ & Type & Note \\
\hline$r+1$ & 0 & 1 & Singlet>doublet & $\begin{array}{l}\text { Radical appears on atom } \\
\text { singlet atom becomes } \\
\text { doublet }\end{array}$ \\
\hline$r-1$ & 1 & 0 & Doublet>singlet & Radical disappears \\
\hline$r+2$ & 0 & 2 & $\begin{array}{lr}\text { Singlet } & \text { (non- } \\
\text { radical) } & >\text { triplet } \\
\text { (biradical) } & \\
\end{array}$ & $\begin{array}{l}\text { Atom becomes biradical } \\
\text { (e.g. carbene) from non- } \\
\text { radical }\end{array}$ \\
\hline$r-2$ & 2 & 0 & $\begin{array}{l}\text { triplet (biradical)> } \\
\text { singlet(non-radical) }\end{array}$ & $\begin{array}{l}\text { Biradical atom is } \\
\text { transformed into one } \\
\text { without radical center }\end{array}$ \\
\hline
\end{tabular}

Some situations:<smiles></smiles>

Dynamic charge:

Atom $1-" c+1 "$

Atom $5-$ - c-1" 
<smiles>CC(C)(C)C1CCCC(C(C)(C)C)[NH+]1[O-]</smiles><smiles>[2H]N1C(C(C)(C)C)CCCC1C(C)(C)C</smiles>

Dynamic charge:

Atom $4-$ "c-1"

Atom $7-$ "c-1"

Dynamic radical:

Atom $7-" r+1 "$

Drawback of a given notation:

In outdated versions of ChemAxon + and - signs need to be screened. If screening is absent then display of the property does not correspond to what we want to see:<smiles>CC(C)(C)C1CCCC(C(C)(C)C)[NH+]1[O-]</smiles>

If it is written actually " $c /-/ 1$ " in the file instead of " $c-1$ " then the drawing is what we expect.<smiles>CC(C)(C)C1CCCC(C(C)(C)C)[NH+]1[O]</smiles>

NOTE! Screening symbols should be avoided in SDF file.

Note! This problem was solved in modern versions of ChemAxon Marvin software and screening is not required for right drawing.

\section{Examples}

Reaction: 

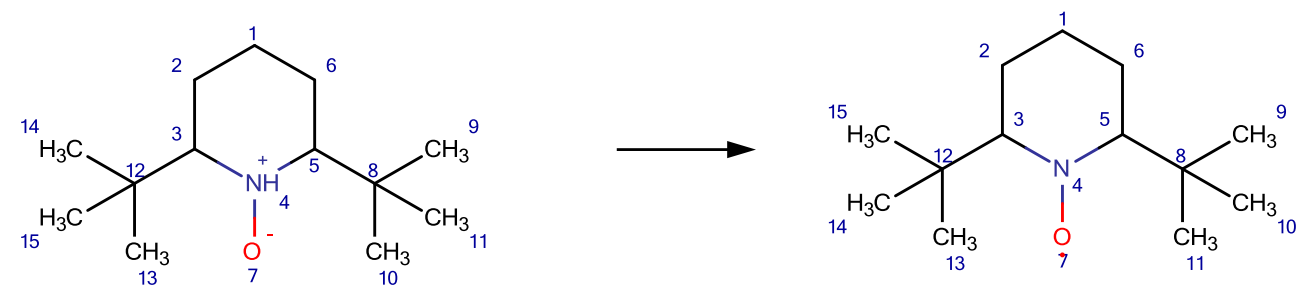

CGR:<smiles>CC(C)(C)C1CCCC(C(C)(C)C)[NH+]1[O]</smiles>

\section{Representation}

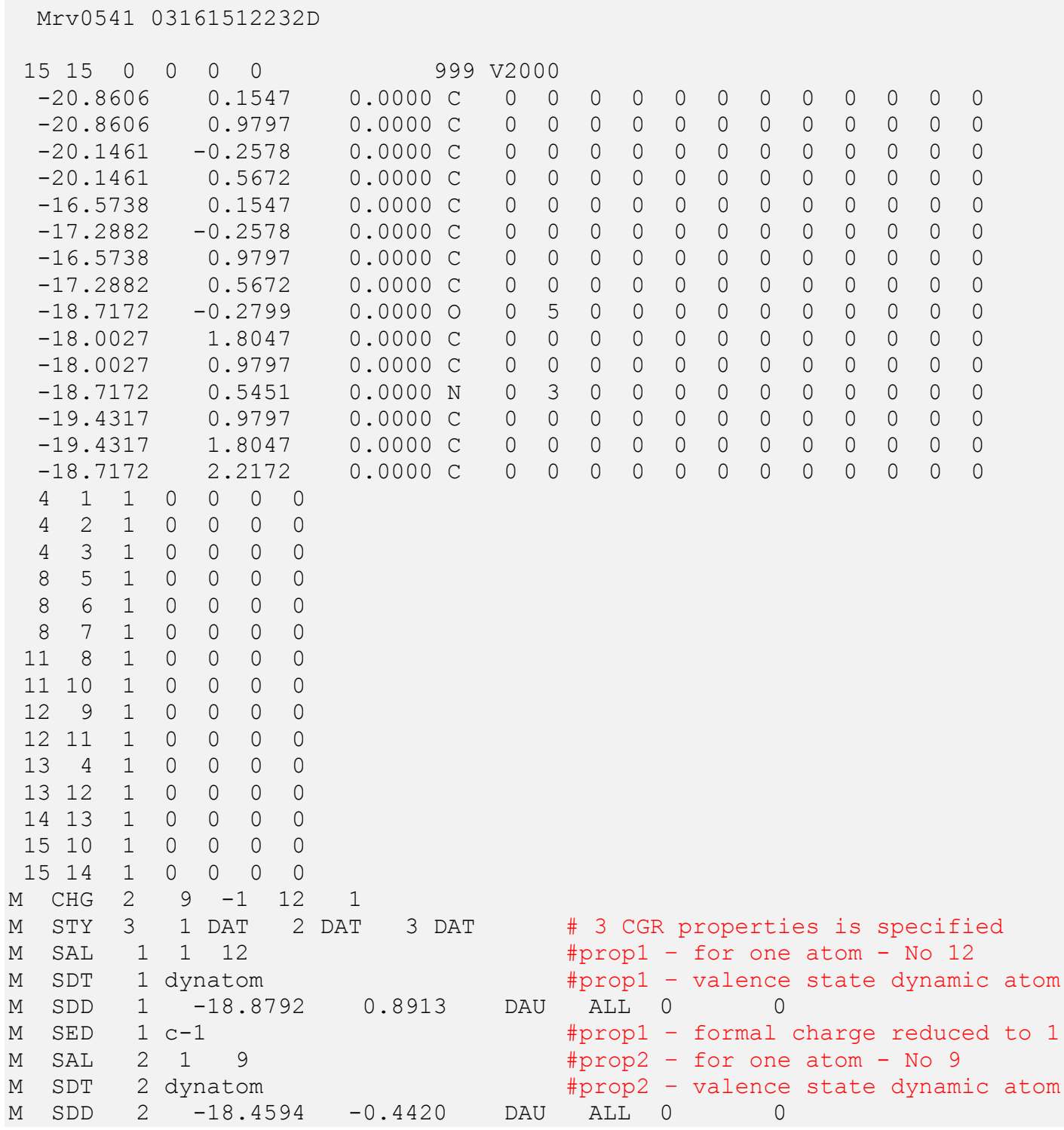




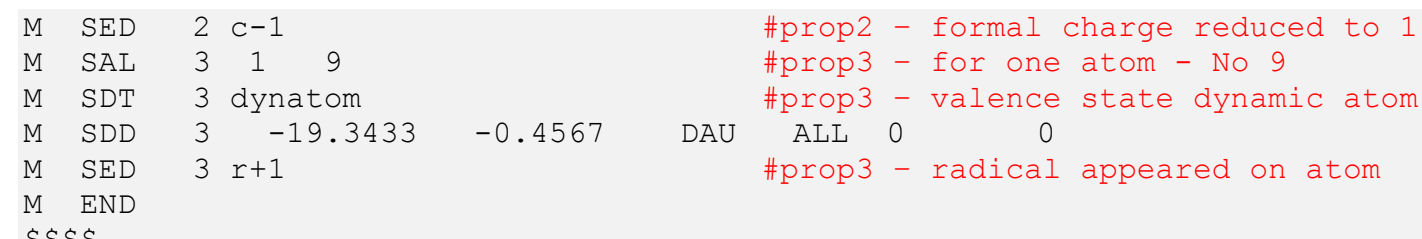




\section{References}

(1) Varnek, A.; Fourches, D.; Horvath, D.; Klimchuk, O.; Gaudin, C.; Vayer, P.; Solov'ev, V.; Hoonakker, F.; Tetko, I. V; Marcou, G. ISIDA - Platform for Virtual Screening Based on Fragment and Pharmacophoric Descriptors. Curr. Comput. Aided-Drug Des. 2008, 4 (3), 191-198. https://doi.org/10.2174/157340908785747465.

(2) Varnek, A.; Fourches, D.; Hoonakker, F.; Solov'ev, V. P.; Solov'ev, V. P.; Solov'ev, V. P. Substructural Fragments: An Universal Language to Encode Reactions, Molecular and Supramolecular Structures. J. Comput. Aided. Mol. Des. 2005, 19 (9-10), 693-703. https://doi.org/10.1007/s10822-005-9008-0.

(3) Lowe, D. M. Extraction of Chemical Structures and Reactions from the Literature, University of Cambridge: Cambridge, 2012, Vol. Ph.D.

(4) James, C. A. OpenSMILES specification http://opensmiles.org/opensmiles.html.

(5) Morgan, H. L. The Generation of a Unique Machine Description for Chemical Structures-A Technique Developed at Chemical Abstracts Service. J. Chem. Doc. 1965, 5 (2), 107-113. https://doi.org/10.1021/c160017a018.

(6) Weininger, D.; Weininger, A.; Weininger, J. L. SMILES. 2. Algorithm for Generation of Unique SMILES Notation. J. Chem. Inf. Model. 1989, 29 (2), 97-101. https://doi.org/10.1021/ci00062a008.

(7) Ihlenfeldt, W. D.; Gasteiger, J. Hash Codes for the Identification and Classification of Molecular Structure Elements. J. Comput. Chem. 1994, 15 (8), 793-813.

https://doi.org/10.1002/jcc.540150802.

(8) Hoonakker, F.; Lachiche, N.; Varnek, A.; Wagner, A. Condensed Graph of Reaction: Considering a Chemical Reaction as One Single Pseudo Molecule . Int. J. Artif. Intell. Tools 2011, 20 (2), 253270.

(9) CTFile Formats. Biovia 2010.

(10) Dalby, A.; Nourse, J. G.; Hounshell, W. D.; Gushurst, A. K. I.; Grier, D. L.; Leland, B. A.; Laufer, J. Description of Several Chemical Structure File Formats Used by Computer Programs Developed at Molecular Design Limited. J. Chem. Inf. Comput. Sci. 1992, 32 (3), 244-255. https://doi.org/10.1021/ci00007a012. 


\section{CGRtools Tutorial}

Notice:

Corresponding Jupyther Notebook (*.ipynb) and required structure files are available at https://github.com/cimm-kzn/CGRtools/tree/master/tutorial 
\% CGRtools Tutorial \% Dr. Ramil Nugmanov; Dr. Timur Madzhidov; Ravil Mukhametgaleev \% Mar 25,2019

\section{Data types and operations with them}

(c) 2019, Dr. Ramil Nugmanov; Dr. Timur Madzhidov; Ravil Mukhametgaleev

Installation instructions of CGRtools package information and tutorial's files see on https://github.com/cimm-kzn/CGRtools

NOTE: Tutorial should be performed sequentially from the start. Random cell running will lead to unexpected results.

In [1]: import pkg_resources

if pkg_resources.get_distribution('CGRtools').version.spl it('.' $)[: 2] !=\left[{ }^{\prime}{ }^{\prime},{ }^{\prime}, 1 '\right]$ :

print('WARNING. Tutorial was tested on 3.1 version of (GRtools')

else:

print ('Welcome!')

Welcome!

In [2]: \# load data for tutorial

from pickle import load

from traceback import format_exc

with open('molecules.dat', 'rb') as $f$ :

molecules = load $(f)$ \# list of MoleculeContainer objec

ts

with open('reactions.dat', 'rb') as $f$ :

reactions $=$ load $(f)$ \# list of ReactionContainer objec

ts

$\mathrm{m} 1, \mathrm{~m} 2, \mathrm{~m} 3, \mathrm{~m} 4=$ molecules \# molecule

$\mathrm{m} 7=\mathrm{m} 3 \cdot \operatorname{copy}()$

$\mathrm{m} 11=\mathrm{m} 3 \cdot \operatorname{copy}()$

m11. standardize()

m7. standardize()

r1 = reactions $[0]$ \# reaction

$\mathrm{m} 5=\mathrm{r} 1$.reactants $[0]$

$\mathrm{m} 8=\mathrm{m} 7$. substructure $([4,5,6,7,8,9]$, as view=False $)$

$\mathrm{m} 10=\mathrm{r} 1 \cdot \operatorname{products}[0] \cdot \operatorname{copy}()$ 
CGRtools has subpackage containers with data structures classes:

- MoleculeContainer - for molecular structure

- ReactionContainer - for chemical reaction

- CGRContainer - for Condensed Graph of Reaction

- QueryContainer - queries for substructure search in molecules

- QueryCGRContainer - queries for substructure search in CGRs

In [3]: from CGRtools.containers import * import all containers

\subsection{MoleculeContainer}

Molecules are represented as undirected graphs. Molecules contain Atom objects and Bond objects.

Atom objects are represented as dictionary with unique number for each atom as key.

Bond objects are stored as sparse matrix with adjacent atoms pair as keys for rows and columns.

Hereafter, atom number is unique integer used to enumerate atoms in molecule. Please, don't confuse it with element number in Periodic Table, hereafter called element number.

Methods for molecule handling and the arguments of MoleculeContainer are described below.

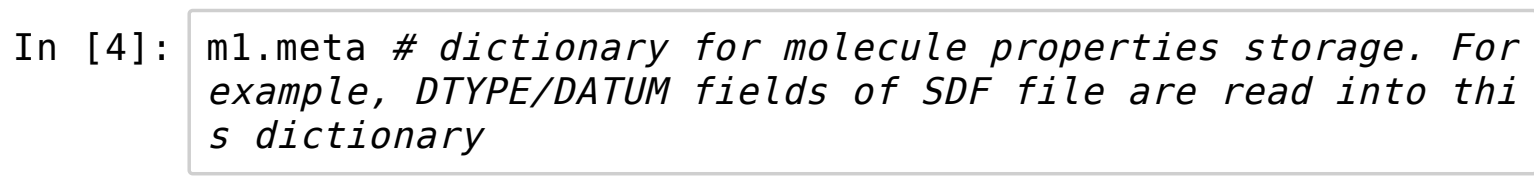

Out [4]: \{'cdid': '001'\}

In [5]: m1 \# MoleculeContainer supports depiction and graphic rep resentation in Jupyter notebooks.

out [5]:<smiles>C=C[AsH3]</smiles> 


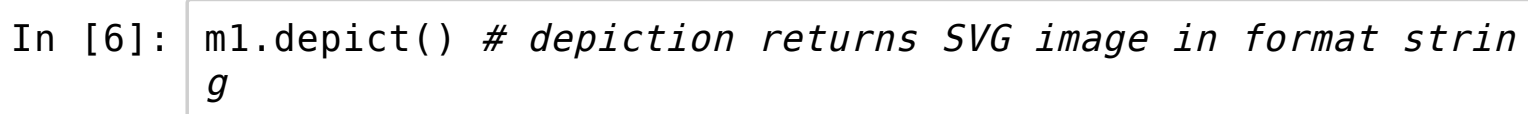

out[6]: '<svg width=" $2.43 \mathrm{~cm}$ " height=" $1.41 \mathrm{~cm} "$ viewBox=" $-11.31-0.6$ $42.431 .41 "$ xmlns="http://www.w3.org/2000/svg" version=" $1.1^{\prime \prime}>\backslash \mathrm{n}<\mathrm{g}$ font-family="sans-serif"> $>\mathrm{n}<\mathrm{g}$ fill="\#FFOD $O D ">\backslash n \quad<$ text $x="-9.52 " y=" 0.00 "$ font-size="0.40" $>0<1$ text $>\backslash n \quad<$ text $x="-9.78^{\prime \prime} y="-0.28 "$ font-size="0.30" $>1$ $5</$ text $>\backslash n \quad</ g>\backslash n</ g>\backslash n<$ fill="none" stroke="blac k" stroke-width $=" .03 ">\backslash n<$ line $x 1="-10.83 "$ y $1="-0.10 "$ $x 2="-10.12 "$ y2="0.31" $/>\backslash n \quad<$ line $x 1="-10.79 "$ y $1="-0.17$ " $\mathrm{x} 2="-10.08 "$ y2="0.24" $/>\backslash n \quad<$ line $x 1="-10.10 "$ y $1=" 0.2$ 8" $\times 2="-9.57 "$ y2=" $-0.03 " />\backslash \mathrm{n} \quad</ \mathrm{g}>\backslash \mathrm{n}</ \mathrm{svg}>$ "

In [7]: with open('molecule.svg', 'w') as $f:$ \# saving image to SV G file f.write (m1.depict ())

In [8]: $\mathrm{m}_{-}$copy $=\mathrm{m} 1$. copy () \# isolated copy of molecule m_copy

Out [8]:

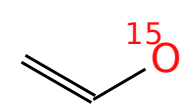

In [9]: len(m1) \# get number of atoms in molecule \# or m1.atoms_count

out [9]: 3

In [10]: ml.bonds_count \# number of bonds

out [10]: 2

In [11]: m1.atoms_numbers \# list of atoms numbers

out $[11]:[3,4,1]$

In [12]: \# this method calculates additional atoms attributes: num ber of neighbors and hybridization. See below for usage m1.reset_query_marks() \# by default this attributes are $N$ one (for speed-up)

m3.reset_query_marks () 
The following notations are used for hybridization of atoms. Values are given as numbers below (in parenthesis symbols that are used in SMILES-like signatures are shown):

- 1 (s) - all bonds of atom are single, i.e. sp3 hybridization

- 2 (d) - atom has one double bond and others are single, i.e. sp2 hybridization

- $3(\mathrm{t})$ - atom has one triple or two double bonds and other are single, i.e. sp hybridization

- 4 (a) - atom is in aromatic ring

Neighbors and hybridizations atom attributes are required for substructure operations and structure standardization. See below

In [13]: \# iterate over atoms using its numbers list(m1.atoms()) \# works the same as dict.items()

Out [13]: [(3, <CGRtools.attributes.molecule.Atom at $0 \times 7 f \odot f 2 b 815048$ $>)$,

(4, <CGRtools.attributes.molecule. Atom at $0 \times 7 f 0 f 2 b 8150 c 8$ $>)$,

$(1,<$ CGRtools.attributes.molecule.Atom at $0 \times 7 f 0 f 2$ b815148 $>$ ) ]

In [14]: \# iterate over bonds using adjacent atoms numbers list (m1. bonds ( ))

Out [14]: [(3, 4, <CGRtools.attributes.molecule.Bond at $0 \times 7 f \circ f 2 b 80 e$ d48>)

$(4,1,<$ CGRtools.attributes.molecule.Bond at $0 \times 7 f 0 f 2$ b $80 e$ d88>) ]

In [15]: \# access to atom by number $\mathrm{m} 1 . \operatorname{atom}(1)$

Out[15]: <CGRtools.attributes.molecule.Atom at 0x7f0f2b815148>

In [16]: try:

m1.atom(10) \# raise error for absent atom numbers except KeyError:

print (format_exc())

Traceback (most recent call last):

File "<ipython-input-16-88aab1feb874>", line 2, in $<\bmod$ ule>

m1.atom(10) \# raise error for absent atom numbers

File "/home/stsouko/cgrtools/lib/python3.7/site-package s/CGRtools/containers/common.py", line 50, in atom return self._node[n]

KeyError: 10

In [17]: \# access to bond using adjacent atoms numbers $\mathrm{m} 1$. bond $(1,4)$

Out[17]: <CGRtools.attributes.molecule.Bond at $0 \times 7 f 0 f 2$ b80ed88> 
In [18]: try:

$\mathrm{m} 1$. bond $(1,3)$ \# raise error for absent bond

except KeyError:

print (format_exc())

Traceback (most recent call last):

File "<ipython-input-18-f820f0aOf50e>", line 2 , in $<\bmod$ ule>

$\mathrm{m} 1$. bond $(1,3)$ \# raise error for absent bond

File "/home/stsouko/cgrtools/lib/python3.7/site-package s/CGRtools/containers/common.py", line 53, in bond return self. adj[n][m]

KeyError: 3

\section{Atom objects are dictinary-like classes which store information about:}

- element

- isotope

- charge

- multiplicity

- xyz coordinates

Also atoms has methods for data integrity checks and include some internally used data.

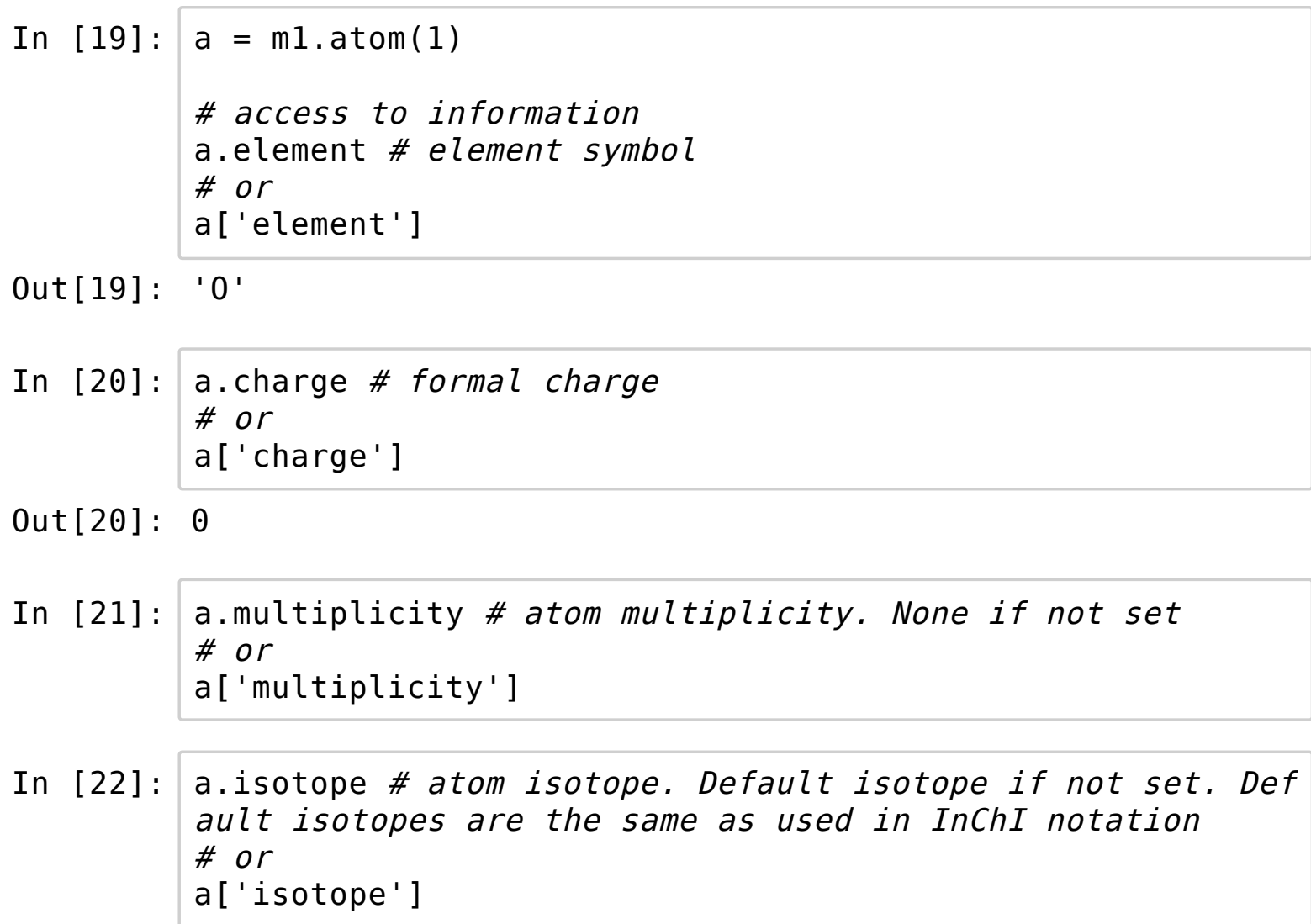

Out [22]: 15 
In [23]: a.x \# coordinates

a.y

a.z

\# or

$\mathrm{a}[\mathrm{\prime} x$ ']

$\mathrm{a}[\mathrm{\prime} y$ ']

$a[' z$ ']

Out [23]: 0.0

In [24]: a.neighbors \# Number of neighboring atoms is calculated $b$ $y$ reset_query_marks method as shown above. It is read-onl $y$.

out [24] : 1

In [25]: a.hybridization \# Atoms hybridization is calculated by re set_query_marks method as shown above. It is read-only.

Out [25] : 1

In [26]: try:

a.hybridization $=2$ \# Not subsettable. Read-only! Thu $s$ error is raised.

except AttributeError:

print (format_exc())

Traceback (most recent call last):

File "<ipython-input-26-baf385e573c1>", line 2, in <mod ule>

a.hybridization $=2$ \# Not subsettable. Read-only! Thu s error is raised.

File "/home/stsouko/cgrtools/lib/python3.7/site-package s/CGRtools/attributes/molecule.py", line 142, in setatt $r$

super()._setattr__(key, value)

AttributeError: can't set attribute

\section{Atomic attributes are subsettable.}

CGRtools has integrity checks for verification of changes induced by user

In [27]: a.isotope $=16$

\# or

a ['isotope'] $=16$ 
In [28]: m1.flush_cache() \# due to caching used for speed-up one $n$ eeds to reset cache of molecule to observe changes in ato ms and bonds

$\mathrm{m} 1$

Out [28] :

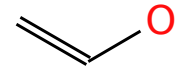

In [29]: try:

a.isotope $=0$ \# raise error. Isotope with mass $\theta$ coul $d$ not exist

except ValueError:

print (format_exc())

Traceback (most recent call last):

File "<ipython-input-29-4063e3b69cod>", line 2, in <mod ule>

d not exist

File "/home/stsouko/cgrtools/lib/python3.7/site-package s/CGRtools/attributes/molecule.py", line 129, in __setatt $r$

value = getattr (self, $f^{\prime}\{$ key $\}$ check') (value)

File "/home/stsouko/cgrtools/lib/python3.7/site-package s/CGRtools/attributes/molecule.py", line 75, in _isotope check

raise ValueError('invalid isotope')

ValueError: invalid isotope

In [30]: \# bond objects also are dictionary-like classes which sto re information about bond order

$b=m 1 . \operatorname{bond}(3,4)$

b. order

\#

$b[$ 'order']

out $[30]: 2$

In [31]: b.order = 1 \# order change also possible

\# or

$\mathrm{b}[$ 'order'] $=1$

In [32]: m1.flush_cache() \#after flushing cashe one could see mole cule with changes $\mathrm{m} 1$

out [32] :<smiles>CCO</smiles> 


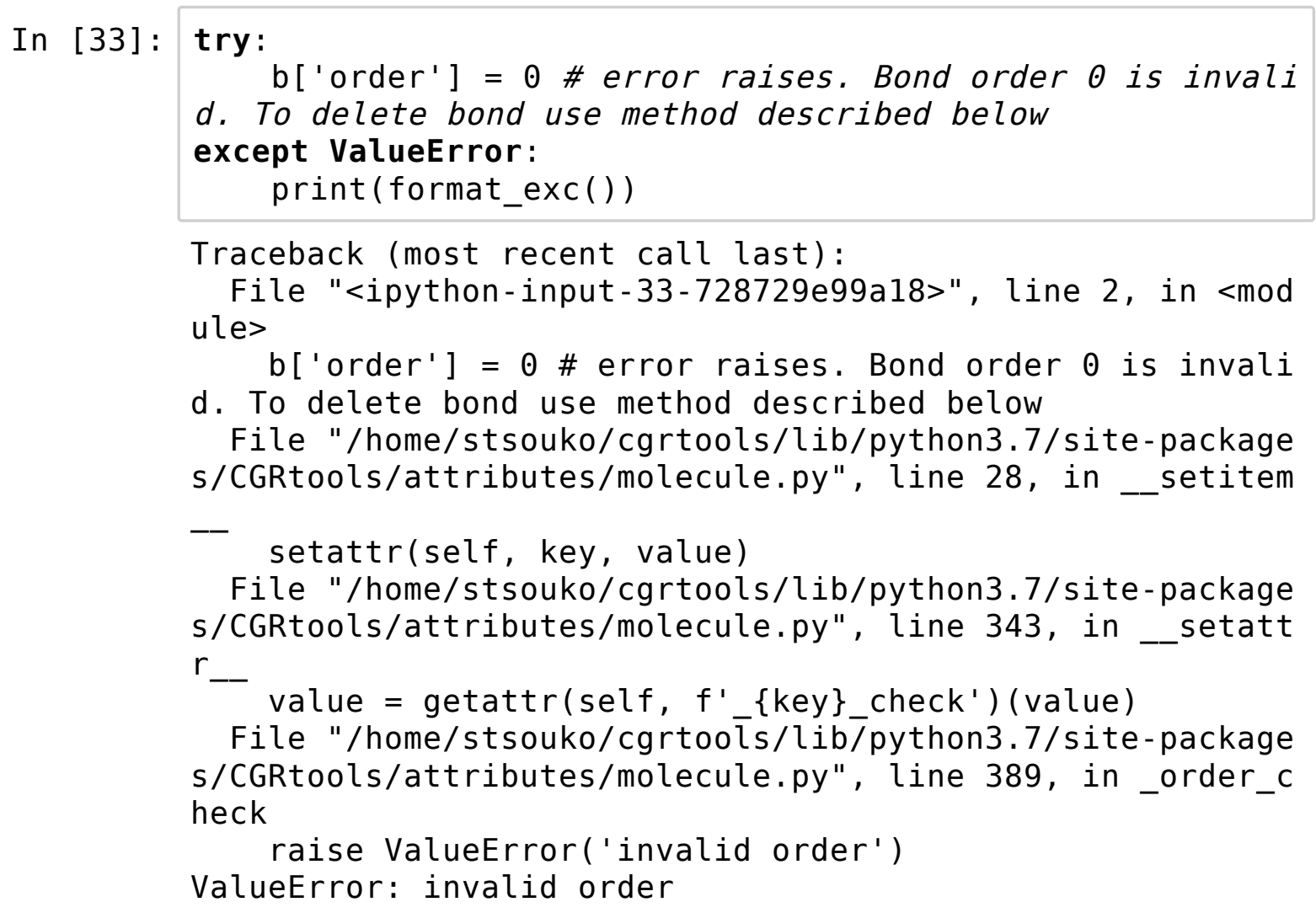

One should to use delete_bond method to break bond

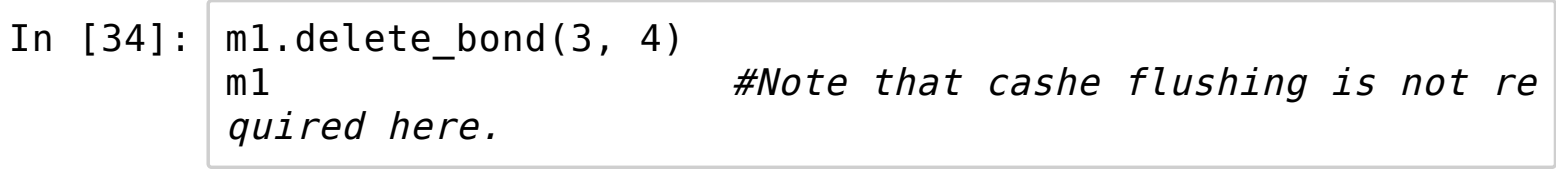

Out [34]:

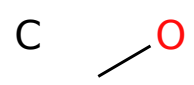

Method delete_atom removes atom from the molecule

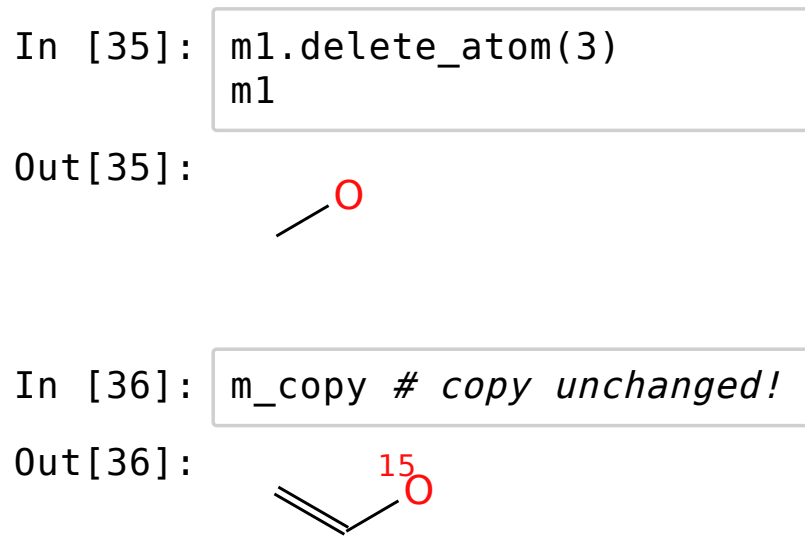


Atoms and bonds objects can be converted into integer representation that could be used to classify their types.

Atom type is represented by 21 bit code rounded to 32 bit integer number:

- 7 bits stand for atom number $\left(2{ }^{* *} 7-1==127\right.$, currently 118 elements are presented in Periodic Table)

- 9 bits are used for isotope (511 posibilities, highest known isotope is $~ 300$ )

- 3 bits stand for formal charge. Charges range from -3 to +3 rescaled to range 0-6

- 2 bits are used for multiplicity.

In [37]: $\operatorname{int}(\mathrm{a})$

\# $131596==0001000 \quad 000010000 \quad 01100$

$\# 0001000==8$ oxygen

\# $000010000==16$ isotope

$\# 011==3(3-3=0)$ uncharged

$\# \Theta \odot==0$ hasn't multiplicity

Out [37] : 131596

In [38]: int(b) \# bonds are encoded by their order

out [38]: 1

In [39]: print(m1.atom_implicit_h(1)) \# get number of implicit hyd rogens on atom 1

print(m1.atom explicit h(1)) \# get number of explicit hyd rogens on atom 1

print(m1.atom_total_h(1)) \# get total number of hydrogens on atom 1

1

0

1

In $[40]: \mathrm{m} 1$

out $[40]$ :

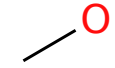

In [41]: m1.check_valence() \# return list of numbers of atoms with invalid valences

out [41]: [ ]

In [42]: m4 \# molecule with valence errors

out [42] :

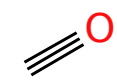


In [43]: m4.check_valence()

out [43] : [1]

In [44]: $\mathrm{m3}$

out [44] :<smiles>O=[N+]([O-])c1ccc(O)cc1</smiles>

In [45]: $\begin{aligned} & \text { m3.sssr \# Method for application of Smallest Set of Small } \\ & \text { est Rings algorithm for rings } \\ & \text { \# identification. Returns list of lists of atoms } \\ & \text { forming smallest rings }\end{aligned}$

Out $[45]$ : $[[4,9,8,7,6,5]]$

\section{Connected components.}

Sometimes molecules has disconnected components (salts etc).

One can find them and split molecule to separate components.

In [46]: $\mathrm{m} 2$ \# it's a salt represented as one graph

out [46] :<smiles>O=C([O-])C(=O)O</smiles>

In [47]: $\begin{aligned} & \text { m2. connected_components \# list of lists of atoms belongin } \\ & g \text { to graph components }\end{aligned}$ out $[47]:[[5,6,7,8,9,10],[11]]$

In [48]: anion, cation = m2.split() \# split molecule to components 
In [49]: anion \# graph of only one salt component

Out [49]:<smiles>O=C([O-])C(=O)O</smiles>

In [50]: cation \# graph of only one salt component

Out [50] : $\quad \mathrm{Na}^{+}$

\section{Union of molecules}

Sometimes it is more convenient to represent salts as ion pair. Otherwise ambiguity could be introduced, for example in reaction of salt exchange:

$\mathrm{Ag}++\mathrm{NO3}-+\mathrm{Na}++\mathrm{Br}-=\mathrm{Ag}++\mathrm{Br}-+\mathrm{Na}++\mathrm{NO3}-$. Reactants and products sets are the same.

In this case one can combine anion-cation pair into single graph. It could be convenient way to represent other molecule mixtures.

In [51]: salt = anion | cation

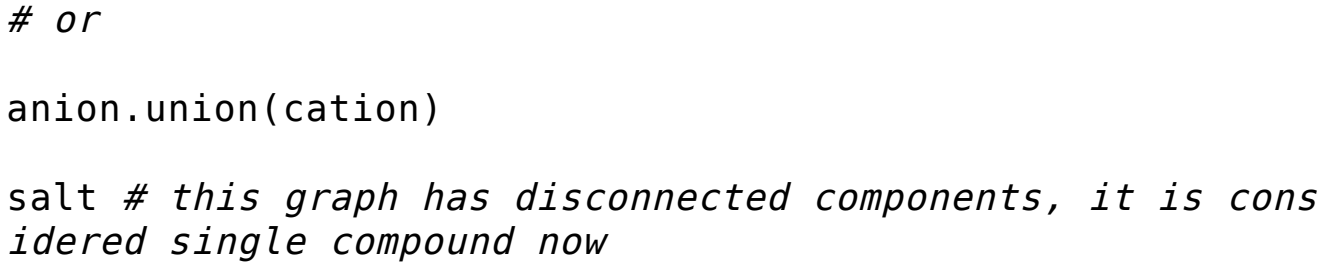

out [51] :<smiles>O=C([O-])C(=O)O</smiles> 


\section{Substructures could be extracted from molecules.}

By default, returned substructures are read-only projections of original molecule (except attributes of atoms/bonds).

Changes in original molecule (bond breaking/formation, atom insertion/deletion, atom/bond attributes changes) will be mirrored in projection.

Projections share the same neighbors and hybridization attributes as in initial molecule even if could be wrong for substructure

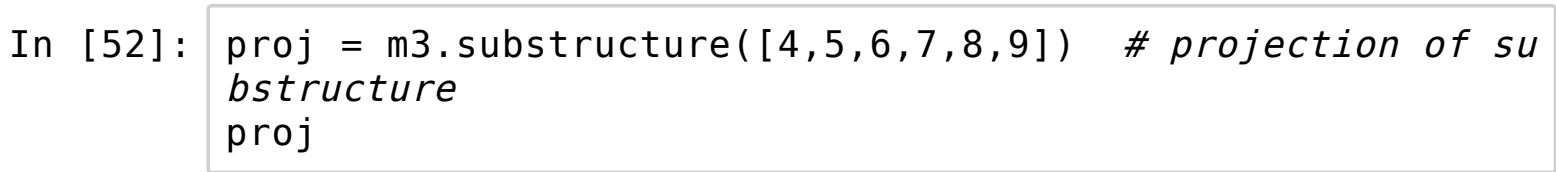

out [52] :<smiles>c1ccccc1</smiles>

In [53]: m3.atom(4).neighbors

out $[53]: 3$

In [54]: proj.atom(4).neighbors \# same as in original molecule and not as it should be in substructure

out $[54]: 3$

In [55]: from networkx.exception import NetworkXError

try:

proj.reset_query marks() \# change of structure for pr ojections is blocked

except NetworkXError:

print (format_exc())

Traceback (most recent call last):

File "<ipython-input-55-cc1c1d2b39f3>", line 3, in $<$ mod ule>

proj.reset_query_marks() \# change of structure for pr ojections is biocked

File "/home/stsouko/cgrtools/lib/python3.7/site-package s/networkx/classes/function.py", line 142, in frozen d")

raise $n x$.NetworkXError("Frozen graph can't be modifie

networkx.exception. NetworkXError: Frozen graph can't be $m$ odified 

In [56]: benzene $=\mathrm{m} 3$. substructure $([4,5,6,7,8,9]$, as_view=False $)$ \# Substructure could be extracted as isolated graph (not pr ojection)
benzene

out [56] :<smiles>c1ccccc1</smiles>
In [57]: benzene.atom(4).neighbors is None \# empty attribute. Subs tructure is a new molecule here. We need to call reset_qu ery_marks

\section{Out [57]: True}

In [58]: benzene.reset_query_marks()

In [59]: benzene.atom(4).neighbors \# now number of neighbors for a tom 4 is 2. It is not 3 as above where projection was use $d$.

out [59]: 2

Note:

- Projection of projection also projection of original molecule

- Projection can be converted to isolated molecule by calling method copy()
In [60]: proj_copy = proj.copy() \# turning projection into molecul e using "copy" method proj_copy

Out [60] :<smiles>c1ccccc1</smiles>

In [61]: proj_copy.reset_query_marks() \# This not a projection any more but a new molecule

Changes in projection are mirrored. See example: 
In [62]: m3.delete_bond $(4,5)$ \# we've deleted bond m3

out [62]:<smiles>C=C/C(O)=C\C=C\[N+](=O)[O-]</smiles>

In [63]: proj.flush_cache() \# remove cached image. Note that in pr ojection the bond is also deleted. proj

out [63] :<smiles>C=C/C=C\C=C</smiles>

augmented_substructure is a substructure consisting from atoms and a given number of shells of neighboring atoms around it. deep argument is a number of considered shells.

It also returns projection by default.
In [64]: aug = m3.augmented_substructure $([10]$, deep=2) \# atom 10 is Nitrogen aug

out [64] :

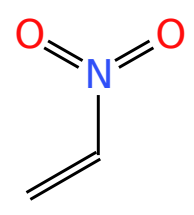

In [65]: aug.atom(10).hybridization \#atom has two incident double bond.

out [65]: 3 


\section{Atoms Ordering.}

This functionality is used for canonic numbering of atoms in molecules. Prime number multiplication based Morgan algorithm is used for atom ranking. Property atoms_order returns dictionary of atom numbers as keys and their ranks according to canonicalization as values. Equal rank mean that atoms are symmetric (are mapped to each other in automorhisms). In present version, instead of sequential ranks prime numbers are returned.

$$
\begin{aligned}
& \text { In [66] : m5.atoms_order } \\
& \text { out [66] : }\{3: 2,1: 3,4: 5\}
\end{aligned}
$$

\section{Atom number can be changed by remap method.}

This method is useful when it is needed to change order of atoms in molecules. First argument to remap method is dictionary with existing atom numbers as keys and desired atom number as values. It is possible to change atom numbers for only part of atoms. Atom numbers could be nonsequencial but need to be unique.

If argument copy is set True new object will be created, else existing molecule will be changed.

Default is False.
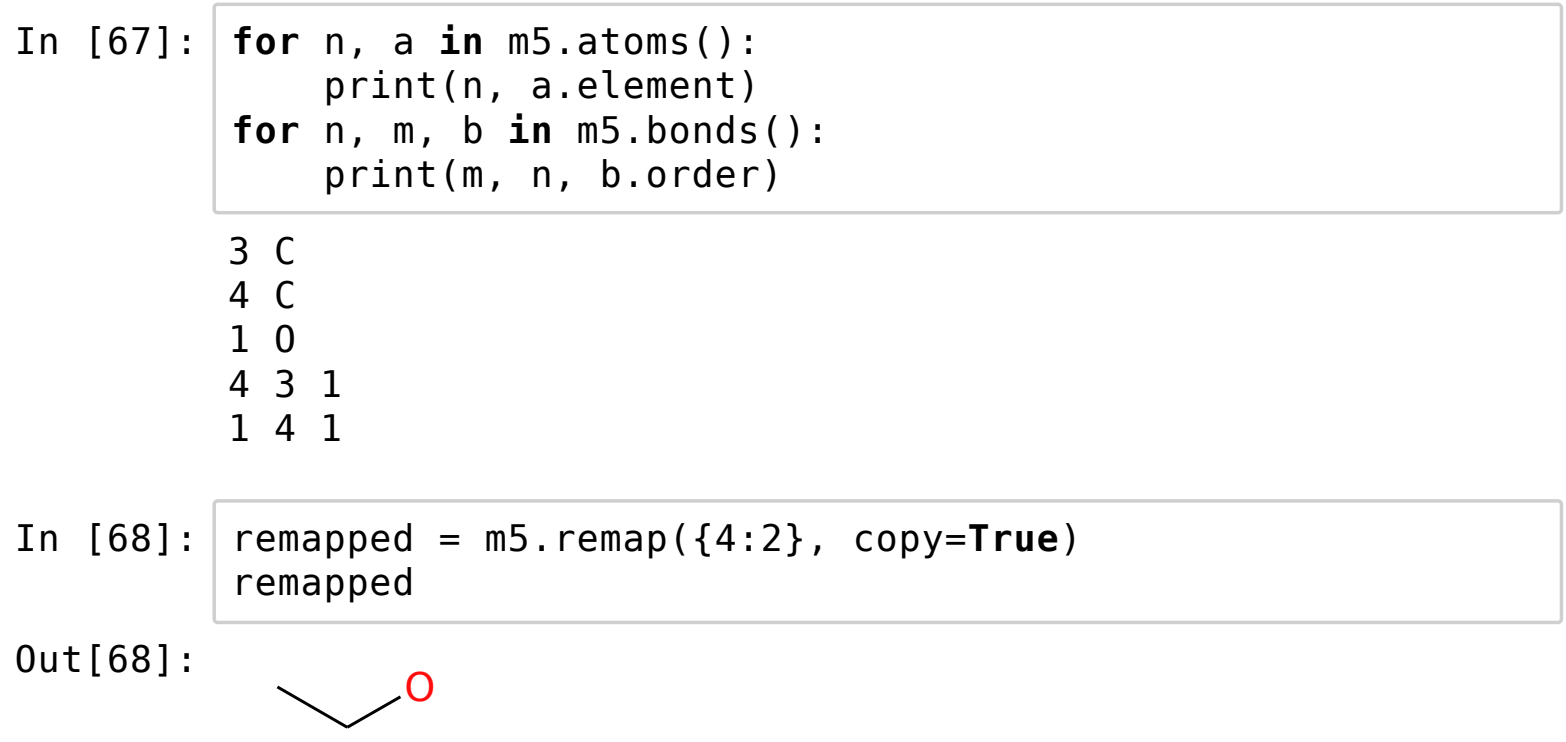

In [69]: for $\mathrm{n}$, a in remapped.atoms ():

print ( $n$, a.element)

for $n, m, b$ in remapped.bonds():

print $(m, n, b . o r d e r)$

$3 \mathrm{C}$

$2 \mathrm{C}$

10

$\begin{array}{lll}2 & 3 & 1\end{array}$

$\begin{array}{lll}1 & 2 & 1\end{array}$ 


\subsection{ReactionContainer}

ReactionContainer objects has the following properties:

- reactants - list of reactants molecules

- reagents - list of reagents molecules

- products - list of products molecules

- meta - dictinary of reaction metadata (DTYPE/DATUM block in RDF)

In [70]: r1 \# depiction supported

out [70] :
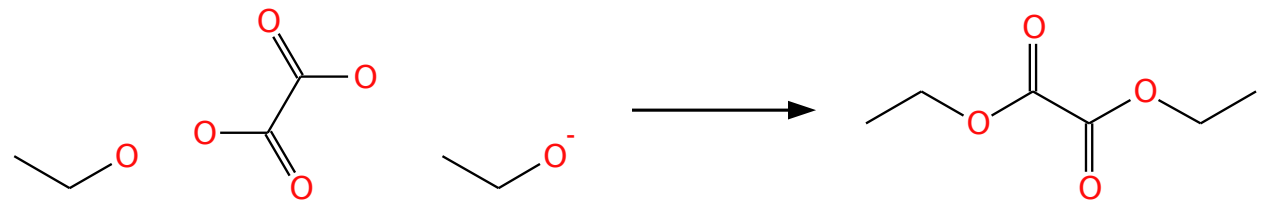

In [71]: r1.meta

Out [71]: \{'CdId': '1872',

'solvent': '3',

'temperature': '129.5',

'tabulated constant': '-6.87'\}

In [72]: print(r1.reactants, r1.products) \# Access to lists of re actant and products. Molecules' signatures are returned $b$ $y$ print() method.

reactant 1 , reactant 2 , reactant $3=$ r1. reactants product $=r 1$. products $[0]$

$[C-C-0,0-C(=0)-C(-0)=0, C-C-[0-]] \quad[C-C-0-C(=0)-C(=0)-0-C$ $-\mathrm{C}]$

Reactions also has standardize, aromatize, reset_query_marks, implicify_hydrogens and explicify_hydrogens methods (see part 3). These methods are applied independently to every molecule in reaction. 


\subsection{CGR}

CGRContainer object is similar to MoleculeConrtainer, except some methods. The following methods are not suppoted for CGRContainer:

- aromatize

- standardize

- implicify_hydrogens

- explicify_hydrogens

- atom_implicit_h

- atom_explicit_h

- atom_total_h

- check_valence

CGRContainer also has some methods absent in MoleculeConrtainer:

- centers_list

- center_atoms

- center_bonds

CGRContainer is undirected graph. Atoms and bonds in CGR has two states: reactant and product.

\section{Composing to CGR}

As mentioned above, atoms in MoleculeContainer have unique numbers. These numbers are used as atom-to-atom mapping in CGRtools upon CGR creation. Thus, atom order for molecules in reaction should correspond to atom-to-atom mapping.

Pair of molecules can be transformed into CGR. Notice that, the same atom numbers in reagents and products imply the same atoms.

Reaction also can be composed into CGR. Atom numbers of molecules in reaction are used as atom-to-atom mapping of reactants to products.

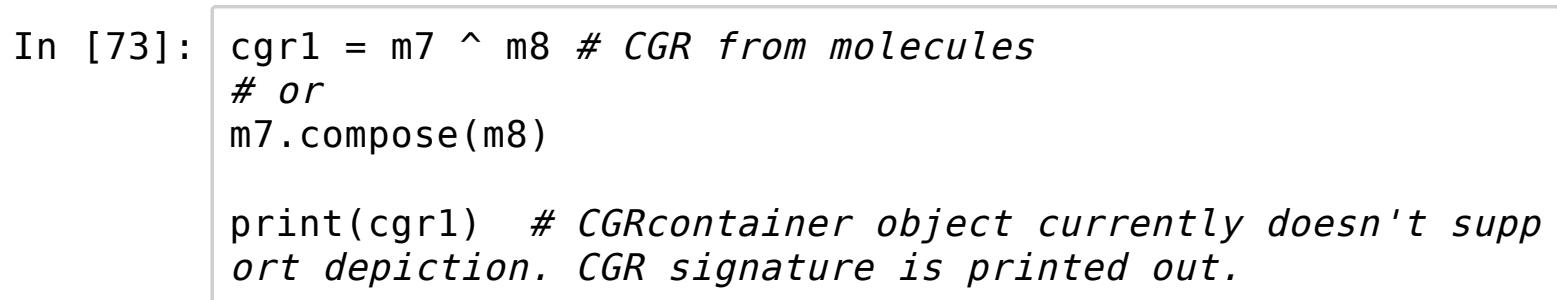

$C: 1: C: C(: C: C: C: 1-[N+](-[0-])=0)-0>>C: 1: C: C(: C: C: C: 1 .[N+]($ $-[0-])=0) .0$ 
This is CGR (depiction is made by ChemAxon externally). You can see changed bonds connected to ring.

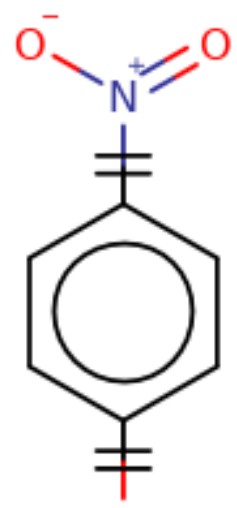

$\mathrm{OH}$

In [74]: $r 1$

Out [74]:

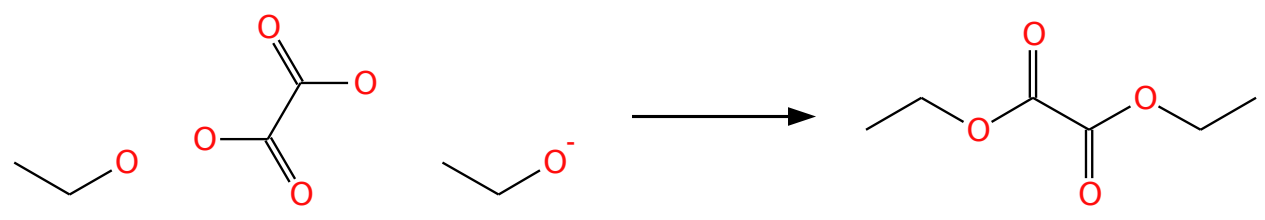

In [75]: $\operatorname{cgr} 2=\sim r 1$ \# CGR from reactions

\# or

r1. compose ()

print(cgr2) \# signature is printed out.

$C-C-[0-] \cdot C(=0)(-0)-C(=0)(-0) \cdot 0-C-C>C-C-0-C(=0)(.0)-C(=0)$ (.0) $-0-C-C$

The history saving thread hit an unexpected error (Operat ionalError('attempt to write a readonly database')). Histo ry will not be written to the database.

It is CGR for reaction (depiction is made by ChemAxon externally).

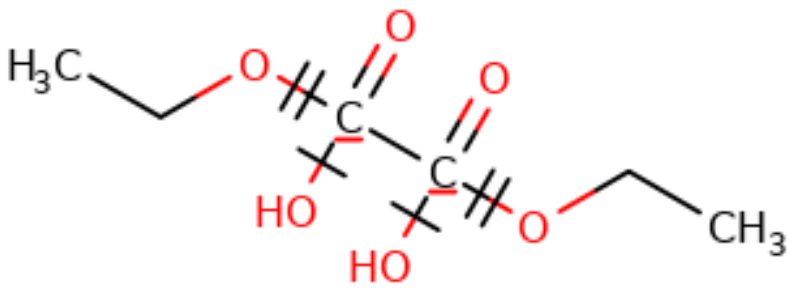

In [76]: cgr2.reset_query_marks() \# CGRs also has reset_query_mark $s$ method 
In [77]: $a=$ cgr2.atom(2) \# atom access is the same as for Molecul eContainer

In [78]: a.element \# element attribute

\# or

a [ 'element']

Out [78]: ' 0 '

In [79]: a.isotope \# isotope attribute

\# or

a [ 'isotope']

Out [79]: 16

For CGRContainer attributes charge, multiplicity, $\mathrm{x}, \mathrm{y}, \mathrm{z}$, neighbors and hybridization refer to atom state in reactant of reaction; arguments $p_{-}$charge, p_multiplicity, p_x, p_y, p_z, p_neighbors and p_hybridization could be used to extract atom state in product part in reaction.

$\begin{array}{ll}\text { In [80]: } & \text { a.charge \# charge of atom in reactant } \\ & \begin{array}{l}\text { or } \\ \text { a['charge'] }\end{array}\end{array}$

out [80]: -1

In [81]: a.p_charge \# charge of atom in product

\#or

a [ 'p_charge']

out [81] : 0

In [82]: a.p_multiplicity \# multiplicity of atom in product. It is None and thus not returned

In [83]: a.p_x \# coordinates of atom in product

a.p_y

a.p_z

Out [83]: 0.0

In [84]: a.neighbors \# number of neighbors of atom in reactant

Out [84] : 1 
In [85]: a.p_neighbors \# number of neighbors of atom in product

out [85]: 2

In [86]: a.hybridization \# hybridization of atom in reactant. 1 me ans only single bonds are incident to atom

out [86] : 1

In [87]: a.p_hybridization \# hybridization of atom in product. $1 \mathrm{~m}$ eans only single bonds are incident to atom

Out [87] : 1

In [88]: $\mathrm{b}=\operatorname{cgrl}$. bond $(4,10)$ \# take bond

\section{Bonds has order and p_order attribute}

If order attribute value is None, it means that bond was formed

If $p \_$order is None, it means that bond was broken

Both order and p_order can't be None

In [89]: b.order \# bond order in reactant

Out [89] : 1

In [90]: b.p_order is None \# bond order in product in None

out [90]: True

CGR can be decomposed back to reaction, i.e. reactants and products.

Notice that CGR can lose information in case of unbalanced reactions (where some atoms of reactant does not have counterpart in product, and vice versa). Decomposition of CGRs for unbalanced reactions back to reaction may lead to strange (and erroneous) structures.

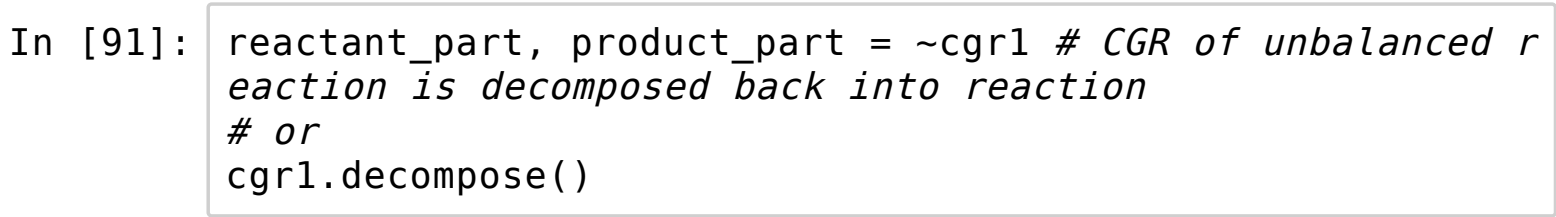

Out [91]: (<CGRtools.containers.molecule.MoleculeContainer at $0 \times 7 \mathrm{f} \odot$ f2b74bef8>, $<$ CGRtools.containers.molecule.MoleculeContainer at $0 \times 7 \mathrm{f} \odot$ $\mathrm{f} 2 \mathrm{~b} 74 \mathrm{bc} 28>$ ) 
In [92]: reactant_part \# reactants extracted. One can notice it is initial molecule

Out [92]:<smiles>O=[N+]([O-])C12CC3CC(O)(C=IC31)C2</smiles>

In [93]: product_part \#extracted products. Originally benzene was the product.

Out [93]:<smiles>O=N[O-]</smiles><smiles>c1ccccc1</smiles>

$\mathrm{O}$

For decomposition of CGRContainer back into ReactionContainer CGRp repa rer class can be used. CGRpreparer is callable object

In [94]: from CGRtools import CGRpreparer \# import of CGRpreparer preparer = CGRpreparer() \# initialization of CGRp reparer

In [95]: decomposed = preparer.decompose(cgr2) \# decomposition of CGR2 into reaction 
In [96]: decomposed \# You can see that water absent in products in itially was restored.

\# This is a side-effect of CGR decomposing that could hel $p$ with reaction balancing.

\# But balancing using CGR decomposition works correctly o nly if minor part atoms are lost

\# but multiplicity and formal charge are saved. In next $r$ elease electronic state balansing will be added.

Out [96] :
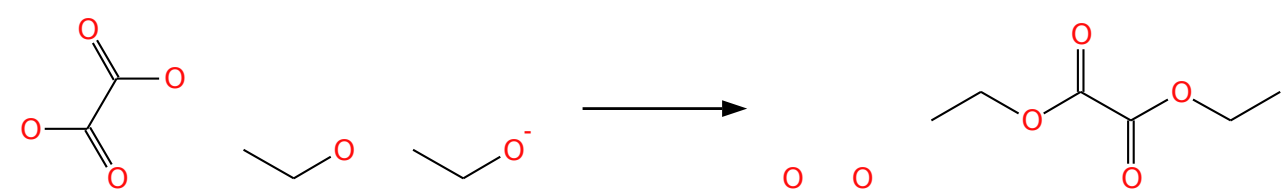

In [97]: r1 \# compare with initial reaction

out [97] :<smiles>CCOCC</smiles><smiles>CCOC(=O)C(=O)OCC</smiles>

\subsection{Queries}

CGRtools supports special objects for Queries. Queries are designed for substructure isomorphism. User can set number of neighbors and hybridization by himself (in molecules they could be calculated but could not be changed).

Queries don't have reset_query_marks method

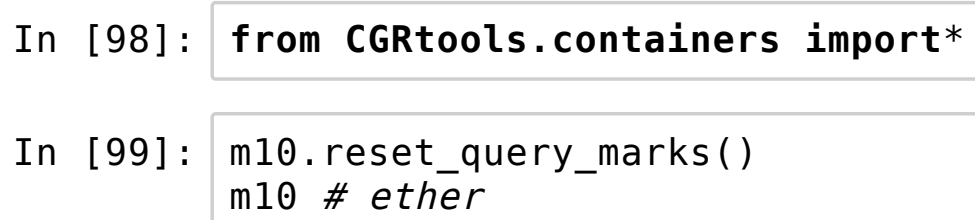<smiles>CCOC(=O)C(=O)OCC</smiles> 

In [100]: carb = m10.substructure([5,7,8, 2]) \# extract projection of carboxyl fragment carb

out [100] :<smiles>O=CO</smiles>

In [101]: q = QueryContainer(carb) \# convert fragment into query print(q) \# QueryContainer don't support depiction yet but signatures can be extracted

$[0 ; s 2 ;]-[C ; d 3 ;]=[0 ; d 1 ;]$

CGRs also can be transformed into Query.

QueryCGRContainer is similar to QueryContainer class for CGRs and has the same API.

QueryCGRContainer take into account state of atoms and bonds in reactant and product, including neighbors and hybridization

\begin{tabular}{|c|c|c|}
\hline$n$ [102]: & $\begin{array}{l}\text { cgrl. reset_query marks() } \\
\text { labels could be calculated } \\
\text { cgr_q = QueryCGRContainer(cgr } 1) \\
\text { into QueryCGRContainer } \\
\text { print (cgr_q) } \\
\text { query }\end{array}$ & $\begin{array}{l}\text { \# cgrl is CGRContaier its } \\
\text { \# transfrom CGRContainer } \\
\text { \# print out signature of }\end{array}$ \\
\hline & $\begin{array}{l}{[\mathrm{C} ; \mathrm{a} 3 ;]: 1(:[\mathrm{C} ; \mathrm{a} 2 ;]:[\mathrm{C} ; \mathrm{a} 2 ;]:[\mathrm{C} ; \mathrm{a} 3} \\
; \mathrm{s} 1 ;])-[\mathrm{N} ; \mathrm{d} 3 ;+](-[0 ; \mathrm{s} 1 ;-])=[0 ; \mathrm{d} 1 \\
; \mathrm{a} 2 ;]:[\mathrm{C} ; \mathrm{a} 2 ;](\mathrm{C} ; \mathrm{C} ; \mathrm{a} 2 ;]:[\mathrm{C} ; \mathrm{a} 2 ;]: 1 \\
1 ;-])=[0 ; \mathrm{d} 1 ;]\end{array}$ & {$[\mathrm{C} ; \mathrm{a} 2 ;]:[\mathrm{C} ; \mathrm{a} 2$} \\
\hline
\end{tabular}

\subsection{Molecules, CGRs, Reactions construction}

CGRtools has API for objects construction from scratch.

CGR and Molecule has methods add_atom and add_bond for adding atoms and bonds.

In [103]: from CGRtools.containers import * 
In [104]: $\mathrm{m}=$ MoleculeContainer() \# new empty molecule

m.add atom(' $\mathrm{C}$ ') \# add Carbon atom using element symbol m.add_atom(6) \# add Carbon atom using element number. \{'element': 6\} is not valid, but \{'element': 'O'\} is also acceptable

m.add atom ( $\{$ 'element': '0', 'charge': -1\}) \# add negative ly chärged oxygen atom. Similarly other atomic properties can be set

\# add_atom has second argument for setting atom number.

\# If not set, the next integer after the biggest among al ready created will be used.

m.add_atom ( \{'element': 'Na', 'charge': 1\}, 4)

Out [104]: 4

In [105]: m.add_bond $(1,2,1)$ \# add bond with order = 1 between ato ms 1 and 2

m.add_bond(3, 2, \{'order': 1\}) \# the other possibility to set bond order

In [106]: m.calculate2d() \#experimental function to calculate atom coordinates. Has number of flaws yet $\mathrm{m}$

Out [106] :<smiles>CCO[C@H]1CO1</smiles>

Reactions can be constructed from molecules

In [107]: $r$ = ReactionContainer() \# empty reaction

r.reactants.append $(\mathrm{m} 1)$ \# add reactant

r.products.append $(\mathrm{m} 11)$ \# add product

\# or

$r=$ ReactionContainer $($ reactant $s=[\mathrm{m} 1]$, product $\mathrm{s}=[\mathrm{m} 11]) \# 0$ ne-step way to construct reaction

\# or

$r=$ ReactionContainer([m1], [m11]) \# first list of Molecu lecontainers is interpreted as reactants, second one - as products 
In [108]: $r$

Out [108] :

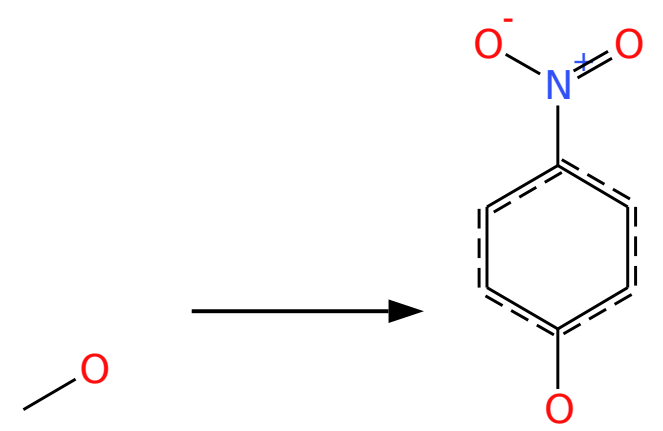

In [109]: \# reactants, products, reagents attributes are list-like. r.products.append(m.copy()) \# One can add (or remove) mol ecules directly to this list of products returned by r.pr oducts

r.flush_cache( )

In [110]: $r$ \# coordinates will left unchanged. Thus depiction could look wrong.

Out $[110]$ :

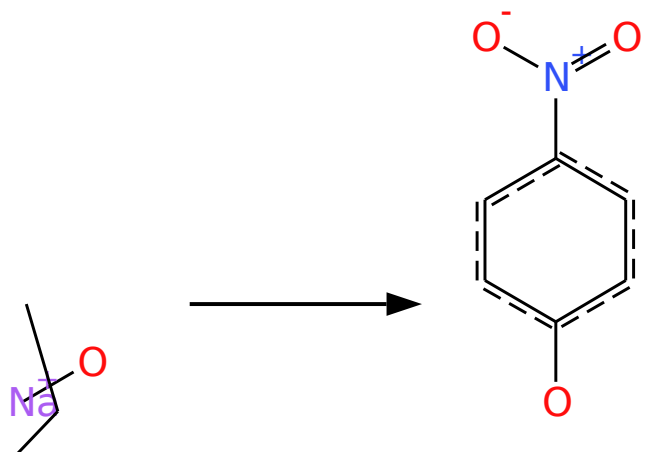

In [111]: r.fix_positions() \# this method fixes coordinates of mole cules in reaction

$r$

Out [111] :

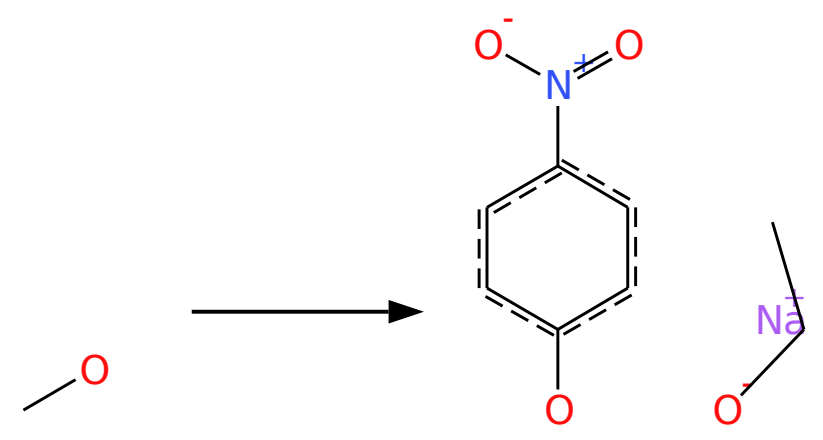


QueryContainers can be constructed in the same way as MoleculeContainers.

Unlike other containers QueryContainers additionally support atoms, neighbors and hybridization lists.

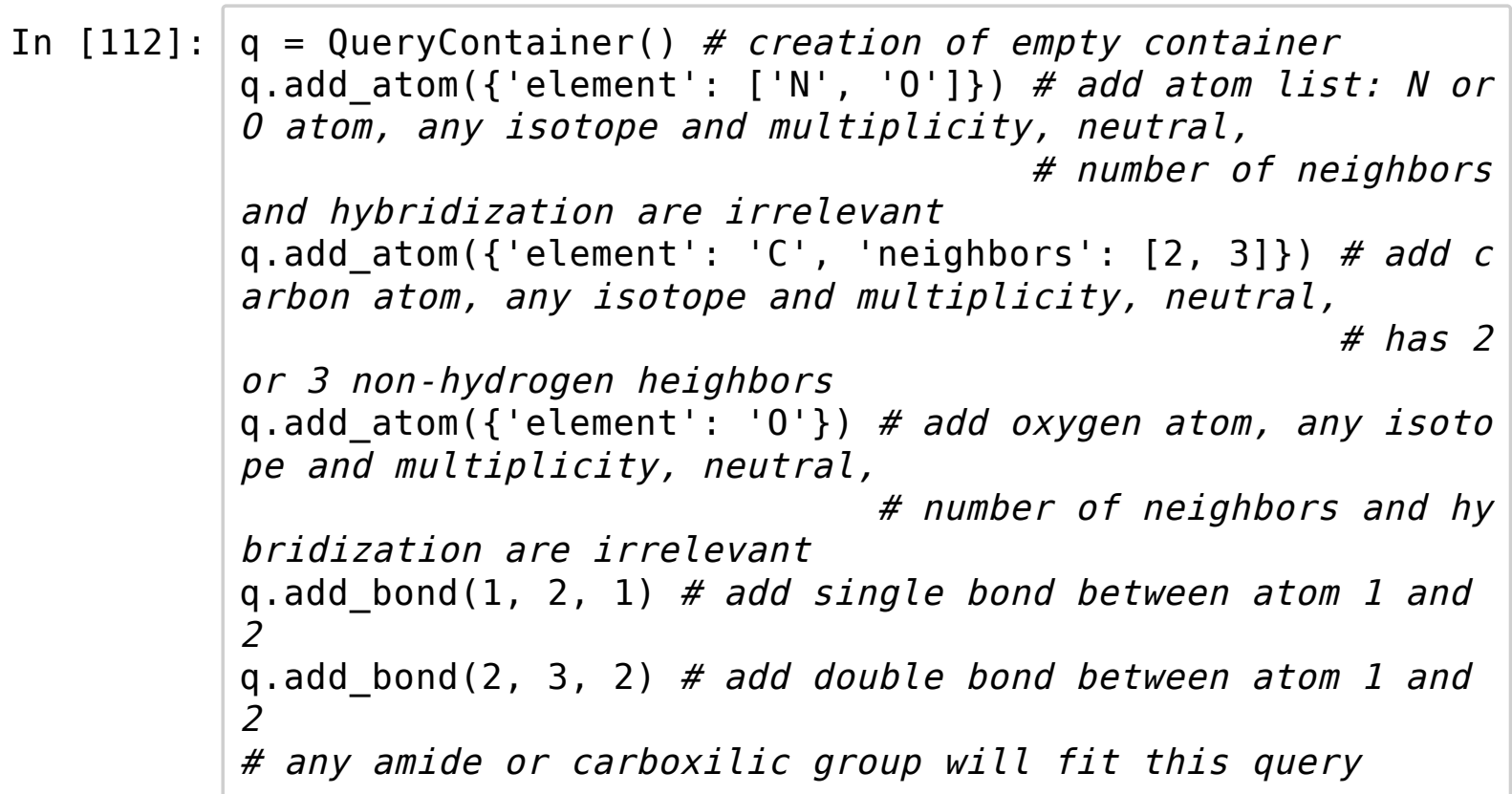

In [113]: print(q) \# print out signature (SMILES-like)

$[N, 0]-[C ;<23>;]=[0]$

\subsection{Extending CGRtools}

You can easily customize CGRtools for your tasks.

CGRtools is OOP-oriented library with subclassing and inheritance support.

As an example, we show how special marks on atoms for ligand donor centers can be added.

In [114]: from CGRtools.containers import MoleculeContainer from CGRtools.attributes import Atom 
In [115]: class MarkedAtom(Atom): \# this class will inherite Atom class lotted! slots__ = '_mark' \# all new attributes should be $s$

def__init__ (self, **kwargs): super()._. init__(**kwargs) attribute self._mark = None \# set default value for added

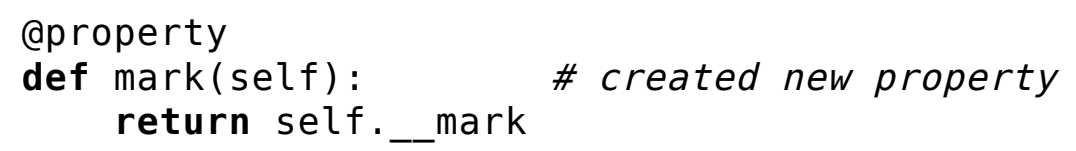

In [116]: class MarkedMoleculeContainer(MoleculeContainer): \# Marke dAtomContainer inherits MoleculeContainer

node_attr_dict_factory = MarkedAtom \# override atom $c$ ontainer

In [117]: $\mathrm{m}=$ MarkedMoleculeContainer() \# create newly developed co ntainer MarkedMoleculeContainer

m.add_atom(' $\mathrm{C}$ ') \# add atom $C$ using methods inherited from MoleculeContainer

m.add_atom ( ' N')

m.add_bond $(1,2,1)$

\# add atom $N$

m.atom(2)['mark'] = 1 \# set mark on atom. In this exampl e dictinary setitem supported but update not.

In [118]: m.atom(2).mark \# one can return mark

Out [118] : 1 


\section{Signatures and duplicates selection}

(c) 2019, Dr. Ramil Nugmanov; Dr. Timur Madzhidov; Ravil Mukhametgaleev

Installation instructions of CGRtools package information and tutorial's files see on https://github.com/cimm-kzn/CGRtools

NOTE: Tutorial should be performed sequentially from the start. Random cell running will lead to unexpected results.

In [1]: import pkg_resources

if pkg_resources.get_distribution('CGRtools').version.spl it('.') [:2] != ['3', '1']:

print('WARNING. Tutorial was tested on 3.1 version of (GRtools')

else:

print ('Welcome!' )

Welcome!

In [2]: \# load data for tutorial

from pickle import load

from traceback import format_exc

with open('molecules.dat', 'rb') as $f$ :

molecules = load $(f)$ \# list of MoleculeContainer objec

ts

with open('reactions.dat', 'rb') as $f$ :

reactions $=$ load $(f)$ \# list of ReactionContainer objec

ts

$\mathrm{m} 1, \mathrm{~m} 2, \mathrm{~m} 3=$ molecules [:3] \# molecule

$\mathrm{m} 7=\mathrm{m} 3 \cdot \operatorname{copy}()$

$\mathrm{m} 11=\mathrm{m} 3 \cdot \operatorname{copy}()$

m11. standardize()

m7. standardize()

r1 = reactions $[0]$ \# reaction

m1.reset_query marks()

m1.flush cache()

m1.deletēatom (3)

$\operatorname{cgr} 2=\sim r \overline{1}$

cgr2.reset_query_marks()

m3. reset_query_marks()

proj $=m \overline{3}$. substructure $([4,5,6,7,8,9])$

benzene $=$ benzene $=\mathrm{m} 3$. substructure $([4,5,6,7,8,9]$, as vie $\mathrm{w}=$ False )

benzene.reset_query_marks()

proj_copy $=$ proj.copy ()

proj_copy.reset_query_marks ()

m3. de lete bond $(\overline{4}, 5)$

proj.flush_cache() 


\subsection{Molecule Signatures}

MoleculeContainer has methods for unique molecule signature generation. Signature is SMILES string with explicit bonds notation and canonical atoms ordering. For pyroles, signatures does not comply with the SMILES rules.

For signature generation one need to call st $r$ function on MoleculeContainer object.

Fixed length hash of signature could be retrieved by calling bytes function on molecule (correspond to SHA 512 bitstring).

Order of atoms calculated by Morgan-like algorithm. On initial state for each atoms it's integer code calculated based on its type. All bonds incident to atoms also coded as integers and stored in sorted tuple. Atom code and tuple of it's bonds used for ordering and similar atoms detecting. Ordered atoms rank is replaced with prime numbers from a prime number lookup table. Atoms of the same type with the same bonds types incident to it have equal prime numbers.

Prime numbers codes found are used in Morgan algorithm cycle.

On each loop for each atom square of its prime number is multiplied to neighboring atoms prime numbers, observed numbers for atoms are ranked and prime numbers are again assigned. Loop is repeated until all atoms will be unique or number of unique atoms will not change in 3 subsequent loops.

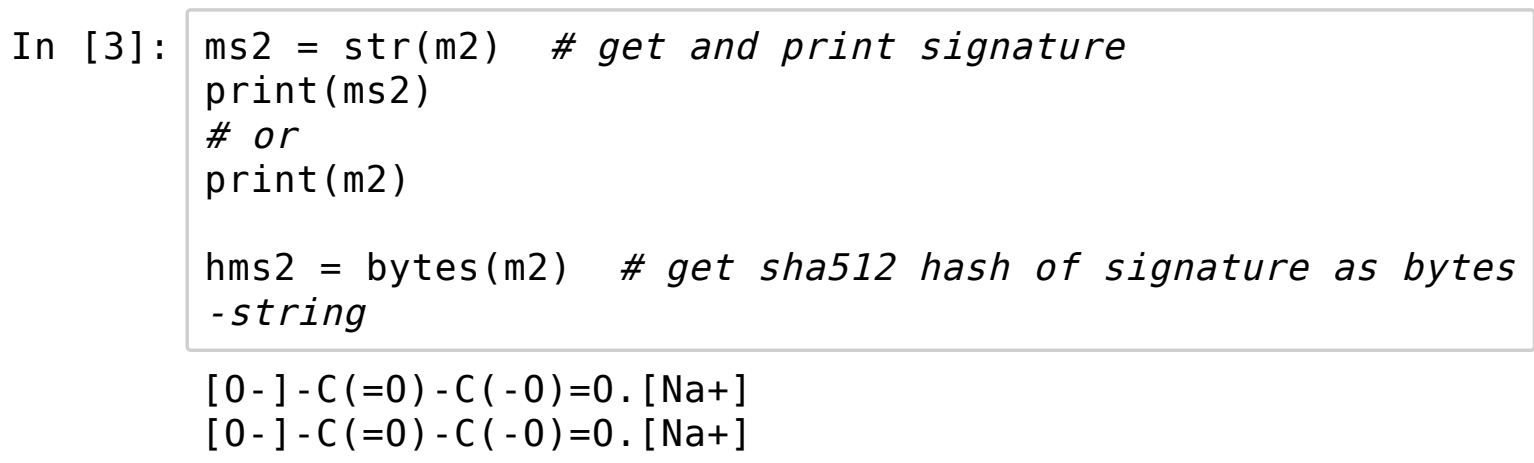

String formatting is supported that is useful for reporting

In [4]: print(f'f string $\left.\{\mathrm{m} 2\}^{\prime}\right)$ \# use signature in string format ting

print ('C-style string $\left.\% s^{\prime} \% \mathrm{~m} 2\right)$

print ('format method \{\} '. format (m2))

$f$ string $[0-]-\mathrm{C}(=0)-\mathrm{C}(-0)=0 .[\mathrm{Na}+]$

$\mathrm{C}$-style string [0-]-C(=0)-C(-0)=0.[Na+]

format method [0-]-C $(=0)-\mathrm{C}(-0)=0$. [ $\mathrm{Na}+]$ 
Number of neighbors and hybridization could be added to signature. Note that in this case they are not readable as SMILES.

For MoleculeContainer and ReactionContainer query marks are not included in signatures and not printed by str and print function. However they could be printed in the following way:

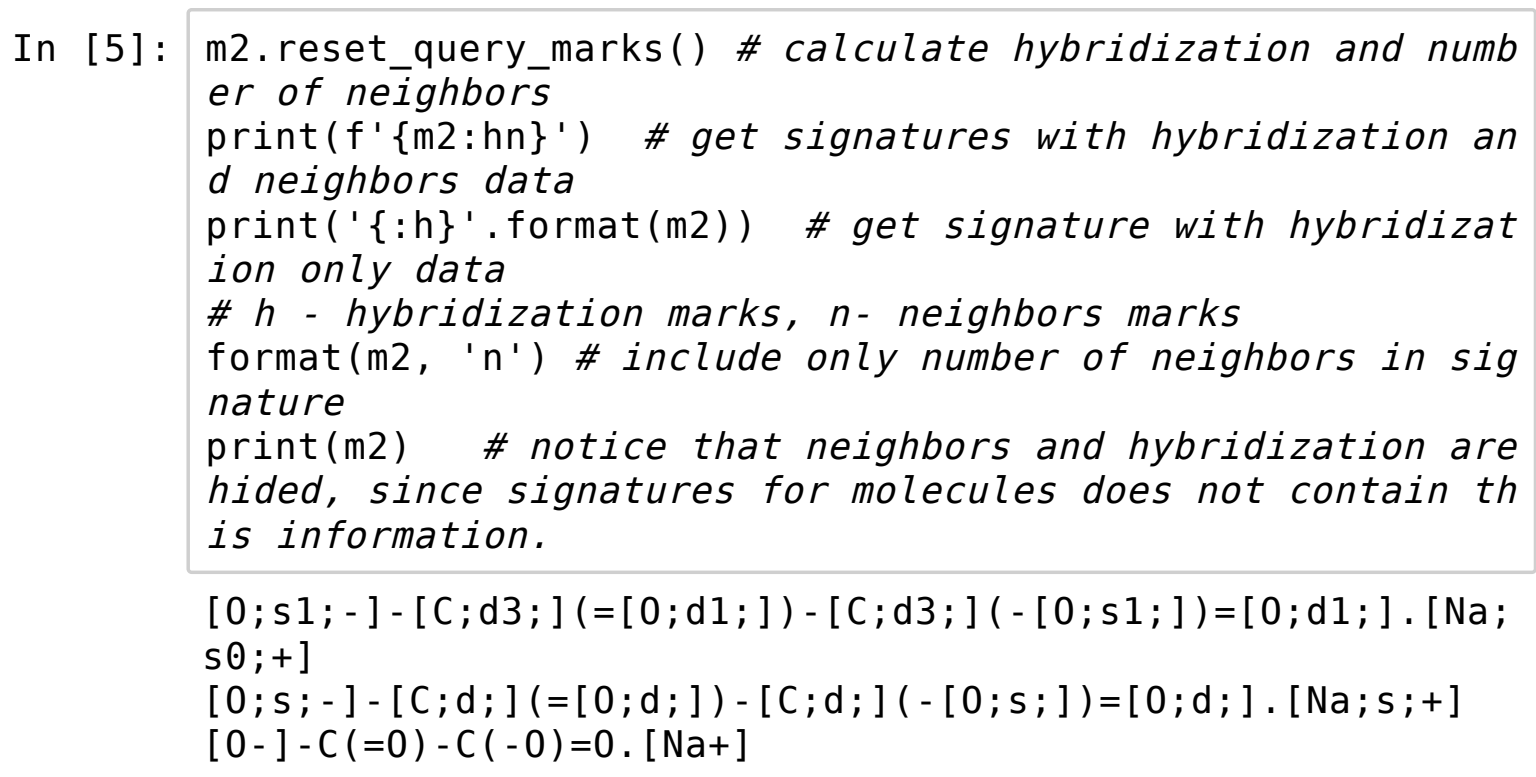

Atoms in the signature are represented in the following way: [element_symbol;hn;charge;multiplicity (if not None)]. $\mathrm{h}$ mean hybridization, $\mathrm{n}$ - number of neighbors. Notation for hybridization is the following:

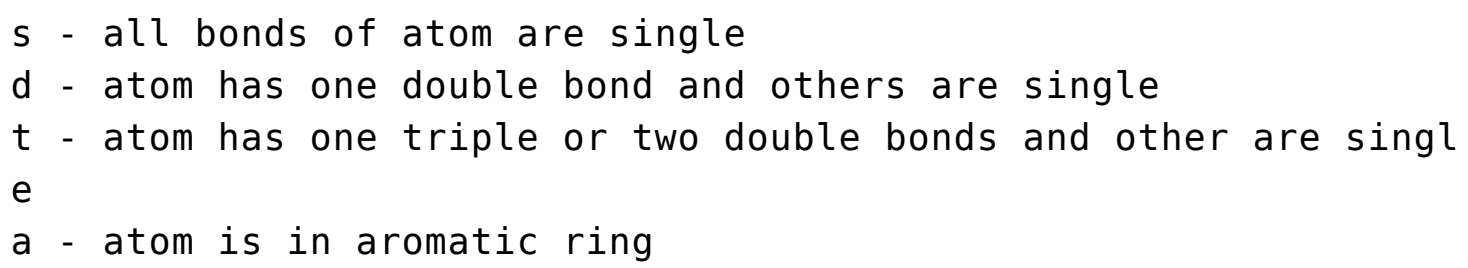

Examples: $\mathrm{s} 1$ - atom has $\mathrm{s}$ hybridization and one neighbor $\mathrm{d} 3$ - atom has $\mathrm{d}$ hybridization and 3 neighbors

Signatures for QueryContainer and QueryCGRContainer include query marks and are printed by str and print function.

Signatures of projections also supported.

$$
\begin{aligned}
& \text { In }[6]: f^{\prime}\{\operatorname{proj}: h\} ' \\
& \text { Out }[6]: \quad '[C ; d ;](=[C ; d ;]-[C ; d ;]=[C ; d ;])-[C ; d ;]=[C ; d ;]^{\prime}
\end{aligned}
$$


Molecules comparable and hashable

Comparison of MoleculeContainer is based on its signatures. Moreover, since strings in Python are hashable, MoleculeContaier also hashable.

NOTE: MoleculeContainer can be changed. This can lead to unobvious behavior of the sets and dictionaries in which these molecules were placed before the change. Avoid changing molecules (standardize, aromatize, hydrogens and atoms/bonds changes) placed inside sets and dictionaries.

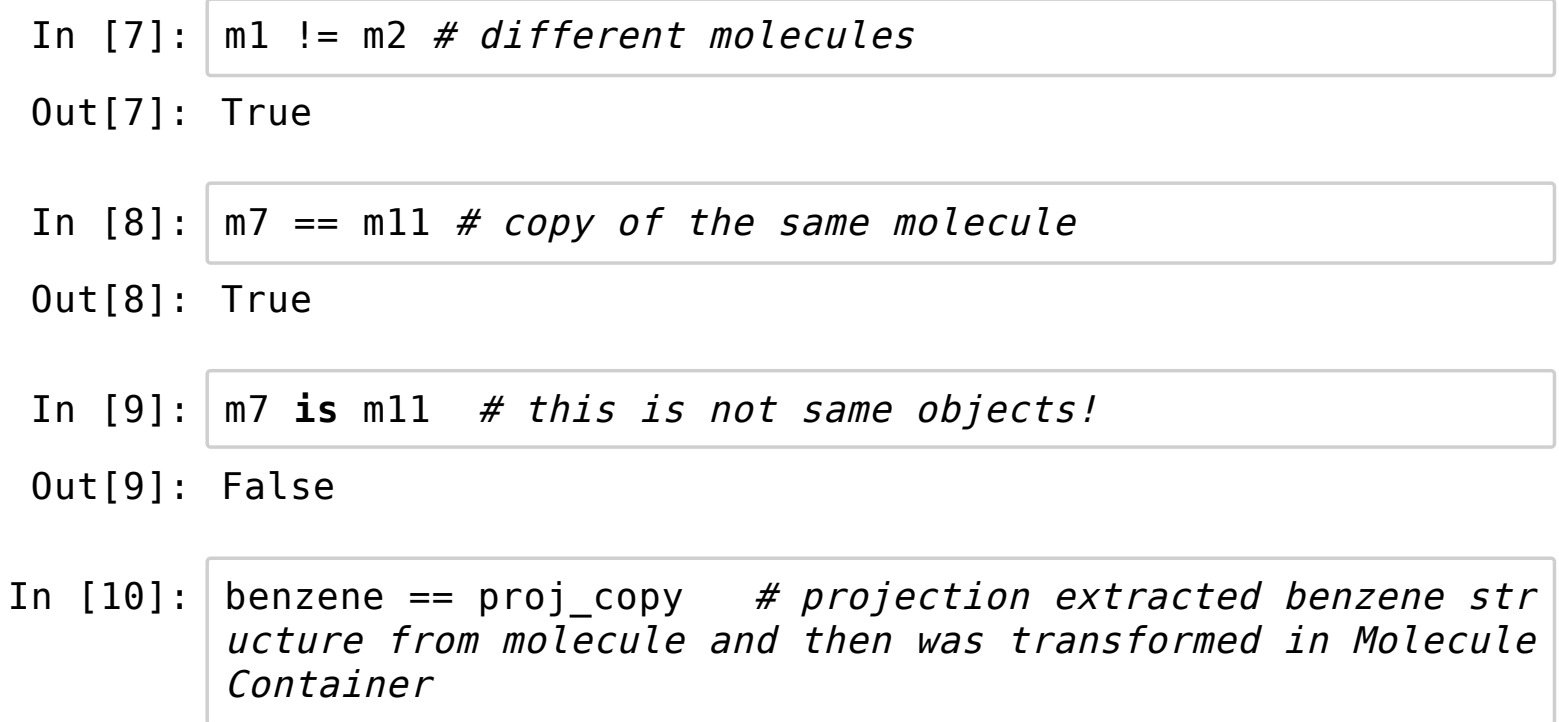

Out [10]: True

In [11]: \# Simplest way to exclude duplicated structures len $(\{\mathrm{m} 1, \mathrm{~m} 2, \mathrm{~m} 7, \mathrm{~m} 11\})==3$ \# create set of unique molecu les. Only 3 of them were different.

Out [11]: True

\subsection{Reaction signatures}

ReactionContainer have its signature. Signature is SMIRKS string in which molecules of reactants, reagents, products presented in canonical order.

API is the same as for molecules

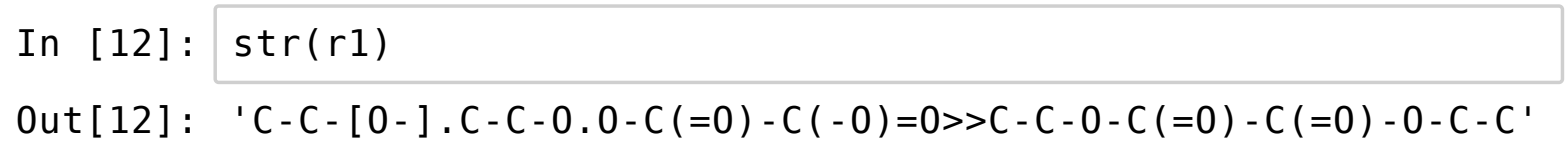

\subsection{CGR signature}

CGRContainer have its signature. Signatures is SMIRKS-like strings where atoms in reactants and products has same order and split by >> symbol 


$$
\begin{aligned}
\text { In [13]: } & \operatorname{str}(\operatorname{cgr} 2) \\
\text { out [13]: } & { }^{\prime} C-C-[0-] . C(=0)(-0)-C(=0)(-0) .0-C-C>C-C-0-C(=0)(.0)-C(=0 \\
& (.0)-0-C-C \text { ' }
\end{aligned}
$$

If one align left- and right-hand side of signature, he will see bond order changes.

$\mathrm{C}-\mathrm{C}-\mathrm{O}-\mathrm{O}-\mathrm{C} \cdot \mathrm{C}(=\mathrm{O})(-\mathrm{O})-\mathrm{C}(=\mathrm{O})(-\mathrm{O}) \cdot \mathrm{O}-\mathrm{C}-\mathrm{C}$ $\mathrm{C}-\mathrm{C}-\mathrm{O}-\mathrm{C}(=\mathrm{O})(. \mathrm{O})-\mathrm{C}(=\mathrm{O})(. \mathrm{O})-\mathrm{O}-\mathrm{C}-\mathrm{C}$ 


\section{Structure standardization}

(c) 2019, Dr. Ramil Nugmanov; Dr. Timur Madzhidov; Ravil Mukhametgaleev

Installation instructions of CGRtools package information and tutorial's files see on https://github.com/cimm-kzn/CGRtools

NOTE: Tutorial should be performed sequentially from the start. Random cell running will lead to unexpected results.

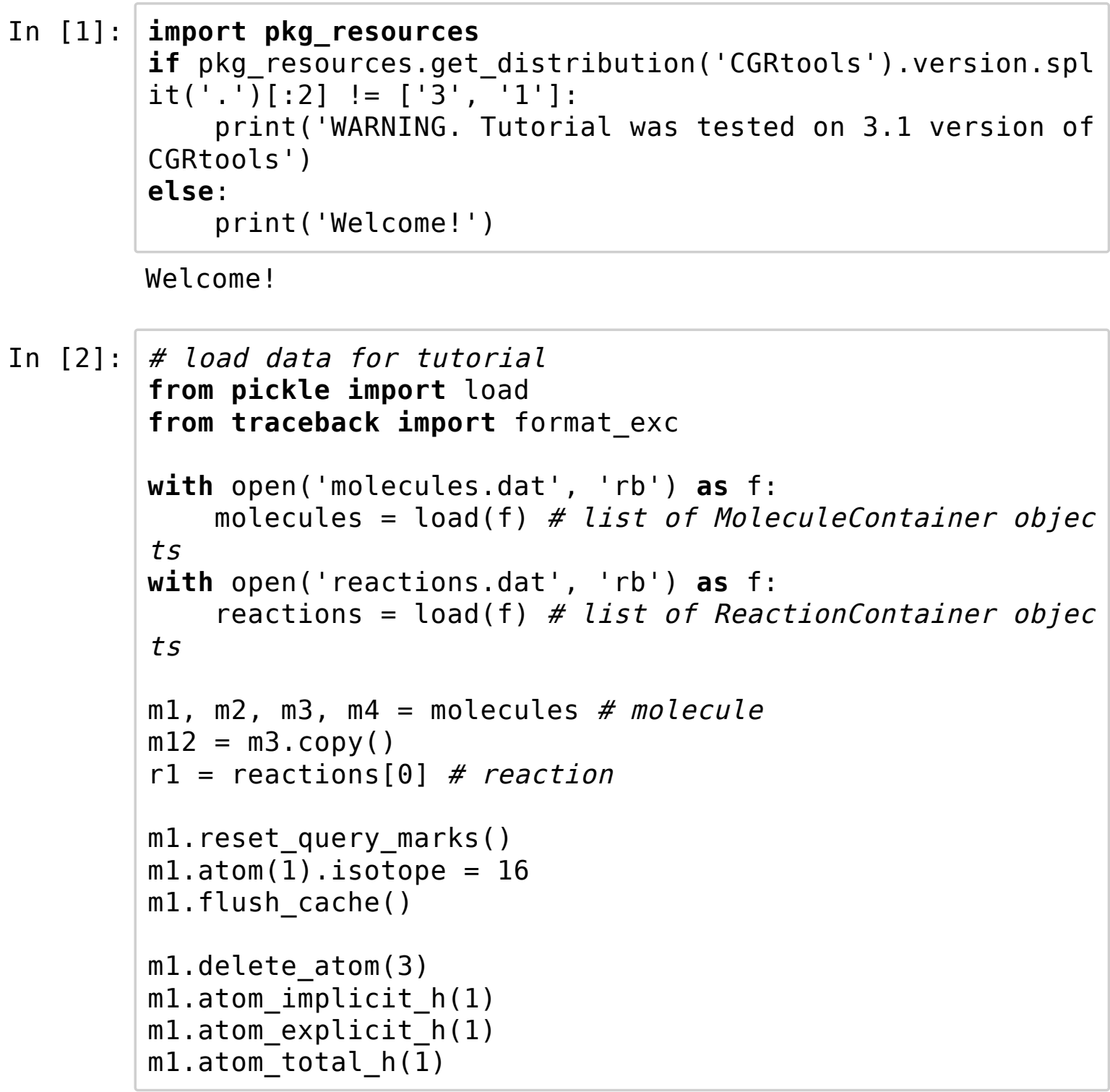

out [2]: 1 


\subsection{Molecules}

MoleculeContainer has standardize and aromatize methods.

Method aromatize transforms Kekule representation of rings into aromatized

Method standardize applies functional group standardization rules to molecules. The following rules are implemented (corresponding SMARTS are given):

- Aromatic N-0xide $[\# 7 ; a: 1]=[0: 2]>>[\# 7+: 1]-[\# 8-: 2]$

- Azide

$[\# 7 ; A ; X 2-: 1][N ; X 2+: 2] \#[N ; X 1: 3]>>[\# 7: 1]=[N+$ :

$2]=[\# 7-: 3]$

- Diazo

$[\# 6 ; X 3-: 1][N ; X 2+: 2] \#[N ; X 1: 3]>>[\# 6 ; A: 1]=[N+:$

2] $=[\# 7-: 3]$

- Diazonium

$[\# 6]-[\# 7: 1]=[\# 7+: 2]>>[\# 6][\mathrm{N}+: 1] \#[\mathrm{~N}: 2]$

- Iminium

$[\# 6 ; X 3+: 1]-[\# 7 ; X 3: 2]>>[\# 6 ; A: 1]=[\# 7+: 2]$

- Isocyanate

$[\# 7+: 1][\# 6 ; A-: 2]=[0: 3]>>[\# 7: 1]=[C: 2]=[0: 3]$

- Nitrilium

$[\# 6 ; A ; X 2+: 1]=[\# 7 ; X 2: 2]>>[C: 1] \#[N+: 2]$

- Nitro

$[0: 3]=[N: 1]=[0: 2]>>[\# 8-: 2]-[\# 7+: 1]=[0: 3]$

- Nitrone Nitronate

$[\# 6 ; A]=[N: 1]=[0: 2]>>[\# 8-: 2]-[\# 7+: 1]=[\# 6$ ;A]

- Nitroso

[\#6] - [\#7H2+:1]- $[\# 8 ; X 1-: 2]>>[\# 6]-[\# 7: 1]=[0: 2]$

- Phosphonic

$[\# 6][P+: 1]([\# 8 ; X 2])([\# 8 ; X 2])[\# 8-: 2]>>[\# 6][P$

:1] ( [\#8]) $([\# 8])=[0: 2]$

- Phosphonium Ylide

] $([\# 6])([\# 6])=[\# 6 ; A: 2]$

- Selenite

$[\# 6][P-: 1]([\# 6])([\# 6])[\# 6+: 2]>>[\# 6][P: 1$

$[\# 8])=[0: 2]$

- Silicate

$14: 1](-[\# 8])=[0: 2]$

- Sulfine

$[\# 6]-[\# 6](-[\# 6])=[S+: 1][\# 8-: 2]>>[\# 6]-[\# 6](-[\# 6]$

)$=[S: 1]=[0: 2]$

- Sulfon

[\#6] $[S ; X 3+: 1]([\# 6])[\# 8-: 2]>>[\# 6][S: 1]([\# 6])$

$=[0: 2]$

- Sulfonium Ylide

$6 ; A: 2]$

- Sulfoxide

$[\# 8 ; \mathrm{X} 2][\mathrm{Se}+: 1]([\# 8 ; \mathrm{X} 2])[\# 8-: 2]>>[\# 8][\mathrm{Se}: 1]($ $[\# 8 ; X 2]-[\# 14+: 1](-[\# 8 ; X 2])-[\# 8-: 2]>>[\# 8]-[\#$ ) $(=[0: 2])=0$

- Sulfoxonium Ylide

$[\# 6][S-: 1]([\# 6])[\# 6+: 2]>>[\# 6][S: 1]([\# 6])=[\#$

$[\# 6][S+: 1]([\# 6])([\# 8-: 2])=0>>[\# 6][S: 1]([\# 6]$

$S: 1]([\# 6])(=[\# 6 ; A])=[0: 2]$

- Tertiary $\mathrm{N}$-0xide

$[\# 6][S+: 1]([\# 6])([\# 8-: 2])=[\# 6 ; A]>>[\# 6][$ :2]

$[\# 6]-[\# 7 ; X 4: 1]=[0: 2]>>[\# 6]-[\# 7+: 1]-[\# 8-$ 
In [3]: m12 \# molecule with kekulized ring

out [3] :<smiles>O=[N+]([O-])c1ccc(O)cc1</smiles>

In [4]: m12.aromatize() \# aromatizes and returns number of transf ormed rings

Out [4]: 1

In [5]: m12 \# cleaned structure. Cache is flushed automatically out [5] :<smiles>O=[N+]([O-])C1CC2CCC(O)(CC2)C1</smiles>

In [6]: m12.standardize() \# apply standardization. Returns numbe $r$ of transformed groups

Out [6]: 1

In [7]: $\mathrm{m} 12$

out [7] :<smiles>O=[N+]([O-])C12CC3CC(O)(C=IC31)C2</smiles> 
Molecules has explicify_hydrogens and implicify_hydrogens methods to handle hydrogens.

This methods is used to add or remove hydrogens in molecule.

Note that currently for pyrole-like molecules implicit hydrogens atoms are calculated incorrectly

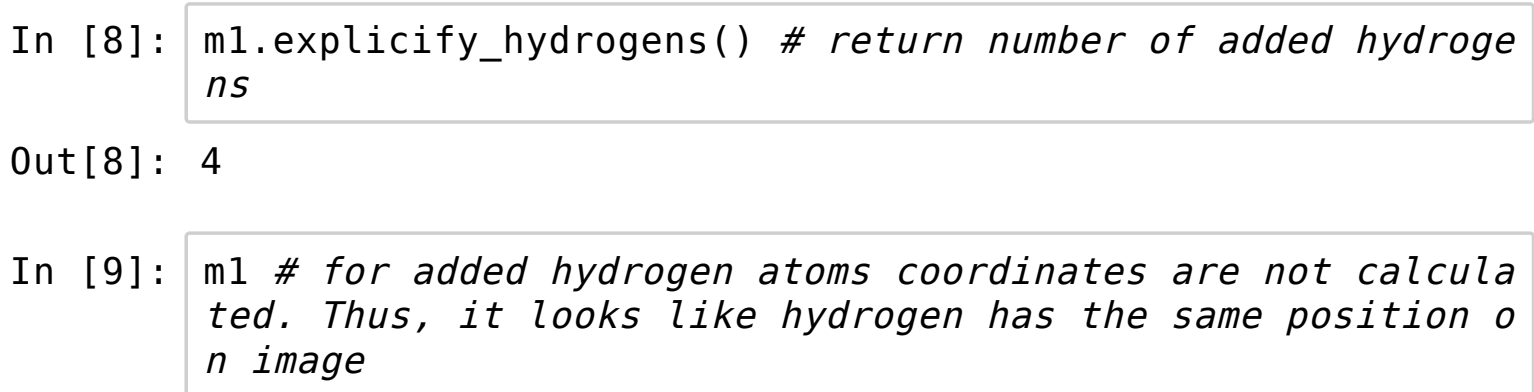

out [9]:

In [10]: m1.implicify_hydrogens()

Out [10]: 4

In [11]: $\mathrm{m} 1$

out [11]:

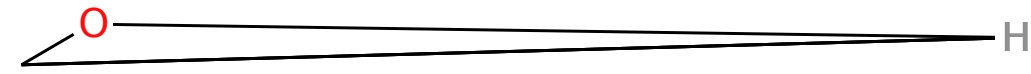

CGRtools has experimental algorithm for $2 \mathrm{~d}$ geometry calcultaion. It works fine only for small molecules. Algorithm requires numpy and scipy packages

In [12]: m1.explicify hydrogens() \# add explicit hydrogens m1. calculate $2 \mathrm{~d}()$ \# experimental force field-based $2 d$ geom etry calculation. $\mathrm{m} 1$

out [12] :<smiles>CO</smiles> 


\subsection{Reactions standardization}

ReactionContainer has standardize, aromatize, explicify_hydrogens and implicify_hydrogens methods that can be applied to reactions. In this case they are applied to all molecules in reaction.

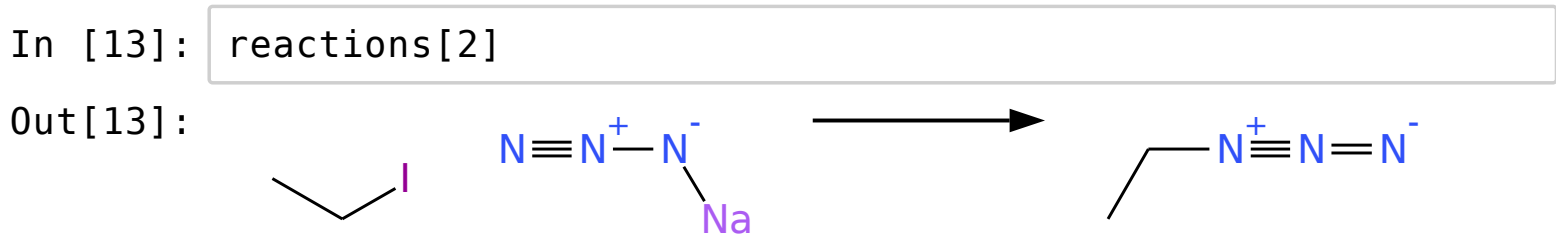
In [14]: reactions [2].standardize() reactions[2].explicify_hydrogens() reactions [2]

Out [14]:

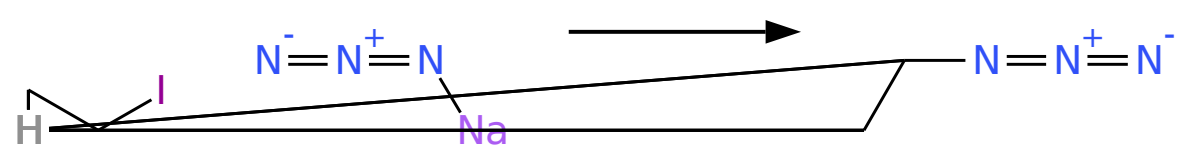




\section{Isomorphism}

(c) 2019, Dr. Ramil Nugmanov; Dr. Timur Madzhidov; Ravil Mukhametgaleev

Installation instructions of CGRtools package information and tutorial's files see on https://github.com/cimm-kzn/CGRtools

NOTE: Tutorial should be performed sequentially from the start. Random cell running will lead to unexpected results.

In [1]: import pkg_resources

if pkg_resources.get_distribution('CGRtools').version.spl it('.') [:2] != ['3', '1']:

print('WARNING. Tutorial was tested on 3.1 version of (GRtools')

else:

print ('Welcome!' )

Welcome!

In [2]: \# load data for tutorial

from pickle import load

from traceback import format_exc

with open('molecules.dat', 'rb') as $f$ :

molecules = load $(f)$ \# list of MoleculeContainer objec ts

with open('reactions.dat', 'rb') as $f$ :

reactions $=$ load $(f)$ \# list of ReactionContainer objec ts

$\mathrm{m} 2, \mathrm{~m} 3=$ molecules $[1: 3]$ \# molecule

$\mathrm{m} 7=\mathrm{m} 3 \cdot \operatorname{copy}()$

m7. standardize ()

$\mathrm{r} 1$ = reactions $[0]$ \# reaction

$\mathrm{m} 5, \mathrm{~m} 6=\mathrm{r} 1$. reactants $[: 2]$

$\mathrm{m} 8=\mathrm{m} 7$. substructure $([4,5,6,7,8,9]$, as_view=False $)$

$\mathrm{m} 9=\mathrm{m} 6$. substructure $([5,6,7,8]$, as_view=Fälse $)$ \# acid

$\mathrm{m10}=\mathrm{r} 1 \cdot \operatorname{products}[0] \cdot \operatorname{copy}()$

benzene $=m 3$. substructure $([4,5,6,7,8,9]$, as_view=False $)$

$\operatorname{cgr} 1=\mathrm{m} 7 \wedge \mathrm{m} 8$

cgrl.reset_query_marks()

carb $=$ m10. substructure $([5,7,8,2])$

m2.reset_query_marks()

from CGRtools. containers import *

from CGRtools import CGRpreparer

preparer $=$ CGRpreparer( $)$ 


\subsection{Molecules Isomorphism}

CGRtools has simple substructure/structure isomorphism API. In backend VF2 algorithm from NetworkX library is used.

Note, that atoms are matched in subgraph isomorphism only if they have same charge/multiplicity and isotope options.

In [3]: $\mathrm{m7}$

out [3]:<smiles>O=[N+]([O-])C1CC2CCC(O)(C=I2)C1</smiles>

In [4]: $\mathrm{m} 8$

out [4]:<smiles>c1ccccc1</smiles>

In [5]: benzene.standardize() benzene

out [5] :<smiles></smiles> 
In [6]:

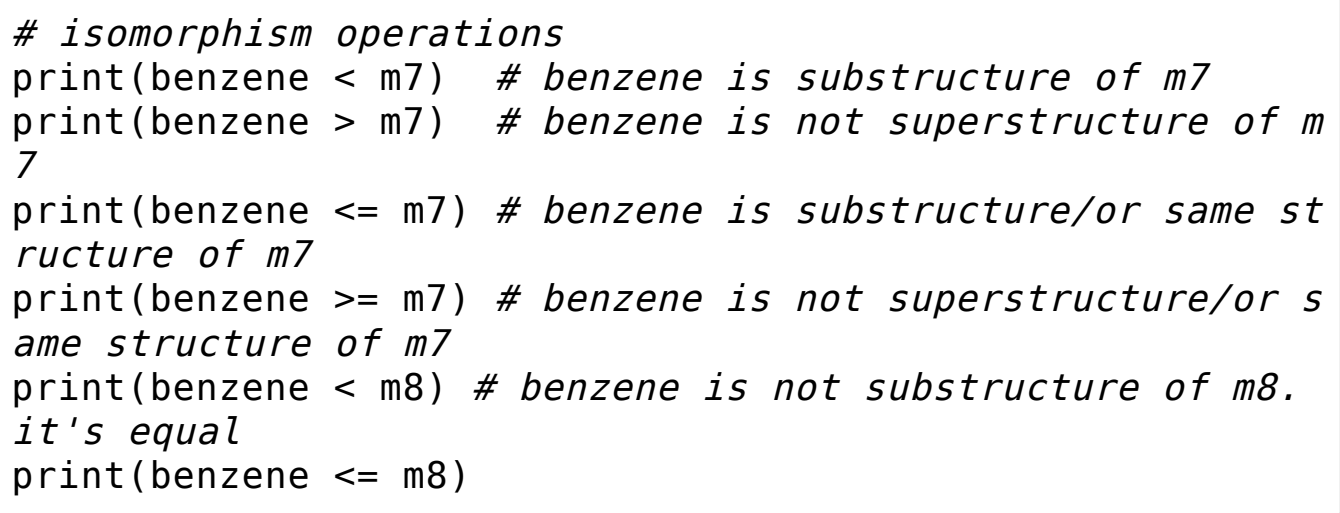

In [7] : $\mathrm{m5}$

out [ 7 ] :

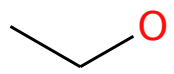

In [8]: $m 6$

out [8] :<smiles>O=C(O)C(=O)O</smiles>

Mappings of substructure to structure can be returned using substructure.get_substructure_mapping(structure, limit=1) method. Argument limit is the number of mappings that one wants to be returned, limit=0 means to return all possible mappings. Method acts as generator.

To get mapping upon structure search structure1.get_mapping(structure2) method was developed. It returns only one possible mapping of all atoms for two isomorphic molecules. This functionality was developed to reorder atoms of two MoleculeContainers in the same order (the dictionary that is given by this method could be directly fed to remap function, see above) for some reaction handling issues. If molecules are isomorphic it works faster than get_substructure_mapping.

$$
\begin{aligned}
& \text { In [9]: } \begin{array}{l}
\text { m5.get_substructure mapping(m6) \# mapping of m5 substruc } \\
\text { ture into m2 superstructure }
\end{array}
\end{aligned}
$$

out $[9]:\{3: 5,4: 6,1: 10\}$ 
In [10]: for $m$ in m5.get_substructure_mapping (m6, limit=0): \# ite rate over all possible substructure mappings print (m)

$\{3: 5,4: 6,1: 10\}$

$\{3: 6,4: 5,1: 8\}$

In [11]: benzene.get_mapping(m8) \# mapping of benzene into m8 - a lso benzene.

Out $[11]:\{4: 4,5: 9,6: 8,7: 7,8: 6,9: 5\}$

\subsection{Reactions}

ReactionContainers do not support isomorphism due to ambiguity. But molecules in reaction can be matched.

In [12]: try: \# it is not possible to match molecule an $d$ reaction. Error is returned $\mathrm{m} 6<\mathrm{r} 1$

except TypeError:

print (format_exc())

Traceback (most recent call last):

File "<ipython-input-12-7ad8567b633a>", line 2, in <mod ule> $\mathrm{m} 6<\mathrm{r} 1$

File "/home/stsouko/cgrtools/lib/python3.7/site-package s/CGRtools/algorithms/isomorphism.py", line 25, in __t __ if len(self) >= len(other):

TypeError: object of type 'ReactionContainer' has no len( )

In [13]: r1.products[0] \# see structure in products

out [13] :<smiles>CCOC(=O)C(=O)OCC</smiles> 
In [14]: $\begin{aligned} & \text { m6 \# substructure used. One can see, they should not matc } \\ & h\end{aligned}$ out [14]:<smiles>O=C(O)C(=O)O</smiles>

In [15]: any (m6 < $m$ for $m$ in $r 1 . p r o d u c t s)$ \# check if any molecule from product side has $m 6$ as substructure

Out [15]: True

\subsection{CGR}

Substructure search is possible with CGRContainer. API is the same as for molecules.

Matching CGR into CGR and molecule into CGR is possible. Note that only conventional bonds in CGR could match moleculear bonds.

Equal atoms in isomorphism is atoms with same charge/multiplicity and isotope numbers in reactant and product states

In [16]: decomposed1 = preparer.decompose(cgr1) \# let's have a loo $k$ at reaction corresponding to $\operatorname{cgr} 1$ decomposed 1

Out [16]:<smiles>O=[N+]([O-])C12CC3CC(O)(C=IC31)C2</smiles><smiles>O=N[O-]</smiles>

O<smiles>c1ccccc1</smiles>

In [17]: m8 \# this's the substructure we are looking for Out [17] :<smiles></smiles> 
In [18]: $m 8<\mathrm{cgr} 1$

out [18]: True

In $[19]: \operatorname{cgr} 1<=\operatorname{cgr} 1$

Out [19]: True

\subsection{Queries}

In [20]: \# to use QueryContainers neighbors and hybridization for molecules need to be calculated

m9.reset_query_marks ()

m10.reset_query_marks()

In [21]: $\mathrm{m} 9$ \# acid

out [21] :

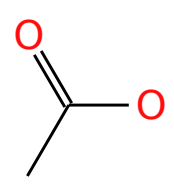

In [22]: m10 \# ether

out [22] :<smiles>CCOC(=O)C(=O)OCC</smiles>

In [23]: carb

out [23] :<smiles>O=CO</smiles>

In [24]: print('m9:', f'\{m9:hn\}') \# all labels were calculated print ('m10:', f'\{m10:hn\}')

print('carb:', f'\{carb:hn\}') \# notice that one of oxygen atom has 2 neighbors. Only ester could fit this restricti on.

$\mathrm{m9:}[\mathrm{C} ; \mathrm{s} 1 ;]-[\mathrm{C} ; \mathrm{d} 3 ;](-[0 ; \mathrm{s} 1 ;])=[0 ; \mathrm{d} 1 ;]$

$\mathrm{m} 10:[\mathrm{C} ; \mathrm{s} 1 ;]-[\mathrm{C} ; \mathrm{s} 2 ;]-[0 ; \mathrm{s} 2 ;]-[\mathrm{C} ; \mathrm{d} 3 ;](=[0 ; \mathrm{d} 1 ;])-[\mathrm{C} ; \mathrm{d} 3 ;](=[$ $0 ; \mathrm{d} 1 ;])-[0 ; \mathrm{s} 2 ;]-[\mathrm{C} ; \mathrm{s} 2 ;]-[\mathrm{C} ; \mathrm{s} 1 ;]$

carb: $[0 ; \mathrm{s} 2 ;]-[\mathrm{C} ; \mathrm{d} 3 ;]=[0 ; \mathrm{d} 1 ;]$ 
Molecules isomorphism don't take into account neighbors and hybridization

In [25]: carb < m9 \# carb currently is molecule projection. It fit this molecule as well.

Out [25]: True

In [26]: carb $<\mathrm{m} 10$ \# carb is a substructure of m10

Out [26]: True

One need to convert molecule (or it's projection) into QueryContainer object. In this case number of neighbors and hybridization data will be taken into account upon substructure search.

API of isomorphism is the same.

In [27]: $q$ = QueryContainer(carb) \# convert molecule into query print (q) \# now one can see that in signature of Query Container. See that one of oxygen has 2 neighbors.

$[0 ; s 2 ;]-[C ; d 3 ;]=[0 ; d 1 ;]$

In [28]: $\mathrm{q}<\mathrm{m} 9$ \# now neighbors and hybridization are taken into a ccount.

Out [28]: False

Acid $\mathrm{m} 9$ has hydroxyl group with one non-hydrogen neighbor. Our query requires existence of one oxygen atom with two non-hydrogen neighbors.

In [29]: q $<10$ \# ester matches to query.

Out [29]: True

In [30]: m2.reset_query_marks( )

$\mathrm{m} 2$

out [30] :<smiles>O=C([O-])C(=O)O</smiles>

In [31]: $\mathrm{q}<\mathrm{m} 2$ \# this molecule does $q$ as substructure as well. It is acid.

Out [31]: False 


\section{Transformation rules extraction}

(c) 2019, Dr. Ramil Nugmanov; Dr. Timur Madzhidov; Ravil Mukhametgaleev

Installation instructions of CGRtools package information and tutorial's files see on https://github.com/cimm-kzn/CGRtools

NOTE: Tutorial should be performed sequentially from the start. Random cell running will lead to unexpected results.

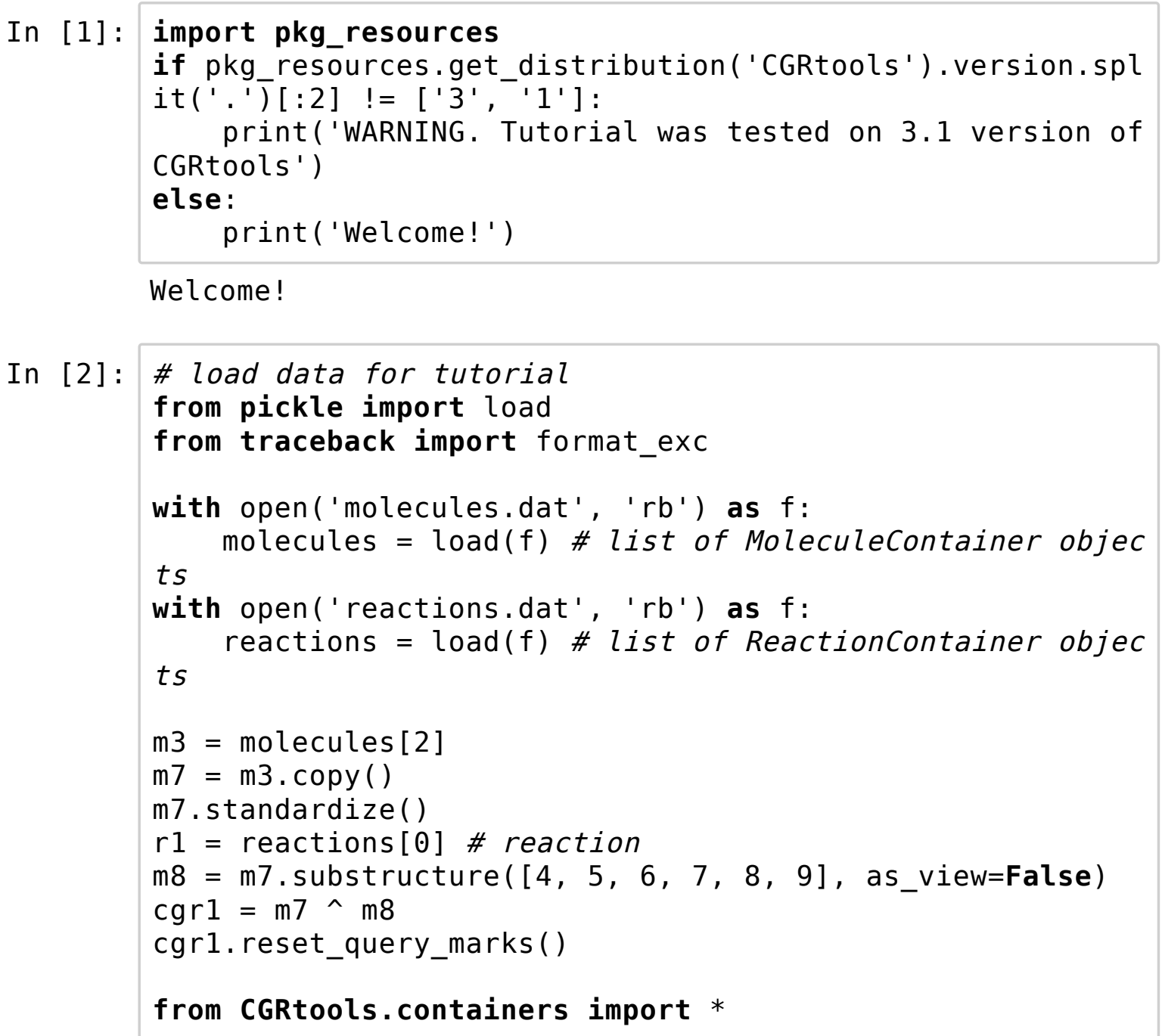

CGRtools can be used to generate molecules and reactions based on a given transformation rule. 

In [3]: cgrl.center_atoms \# list of atom numbers of reaction cent er. If several centers exist they will also be added to $t$ his list.
Out $[3]:[10,4,13,7]$
In [4]: cgrl.center_bonds \# list of dynamic bonds as tuples of ad jacent atom numbers
Out $[4]: \quad[(10,4),(13,7)]$
In [5]: cgrl.centers_list \# list of lists of atom numbers belongi ng to each reaction center.
$t$ into separate lists
\# Distant reaction centers will be spli

Out $[5]: \quad[[10,4],[13,7]]$

In [6]: $\quad r c 1=$ cgrl.substructure $([13,7])$ \# get reaction center fr om CGR

format(rc1, 'hn') \# Notice that query marks are set.

Out $[6]: \quad '[C ; a 3 ;]-[0 ; s 1 ;]>>[C ; a 2 ;] .[0 ; s 0 ;] '$

rc1 is phenol reduction, phenol is transformed into unsubstituted benzene:

- [C;a3;]-aromatic carbon with 3 neighbors

- [0;s1;]-oxygen with 1 neighbor

- [C;a2;]-carbon with 2 neighbors

- [O;s0;]-oxygen without neighbors (water). It probably appears since initial reaction was unbalanced.

In [7]: rule = QueryCGRContainer( $r c 1)$ \# transform reaction into $q$ uery to take query

In [8]: print(rule) \# all query marks are on their place. Without them generation will be too unrestrictive.

\# If needed CGRtools could be used to include atomic environment, etc...

$[\mathrm{C} ; \mathrm{a} 3 ;]-[0 ; \mathrm{s} 1 ;]>>[\mathrm{C} ; \mathrm{a} 2 ;] .[0 ; \mathrm{s} 0 ;]$ 


\section{Reactor}

(c) 2019, Dr. Ramil Nugmanov; Dr. Timur Madzhidov; Ravil Mukhametgaleev

Installation instructions of CGRtools package information and tutorial's files see on https://github.com/cimm-kzn/CGRtools

NOTE: Tutorial should be performed sequentially from the start. Random cell running will lead to unexpected results.

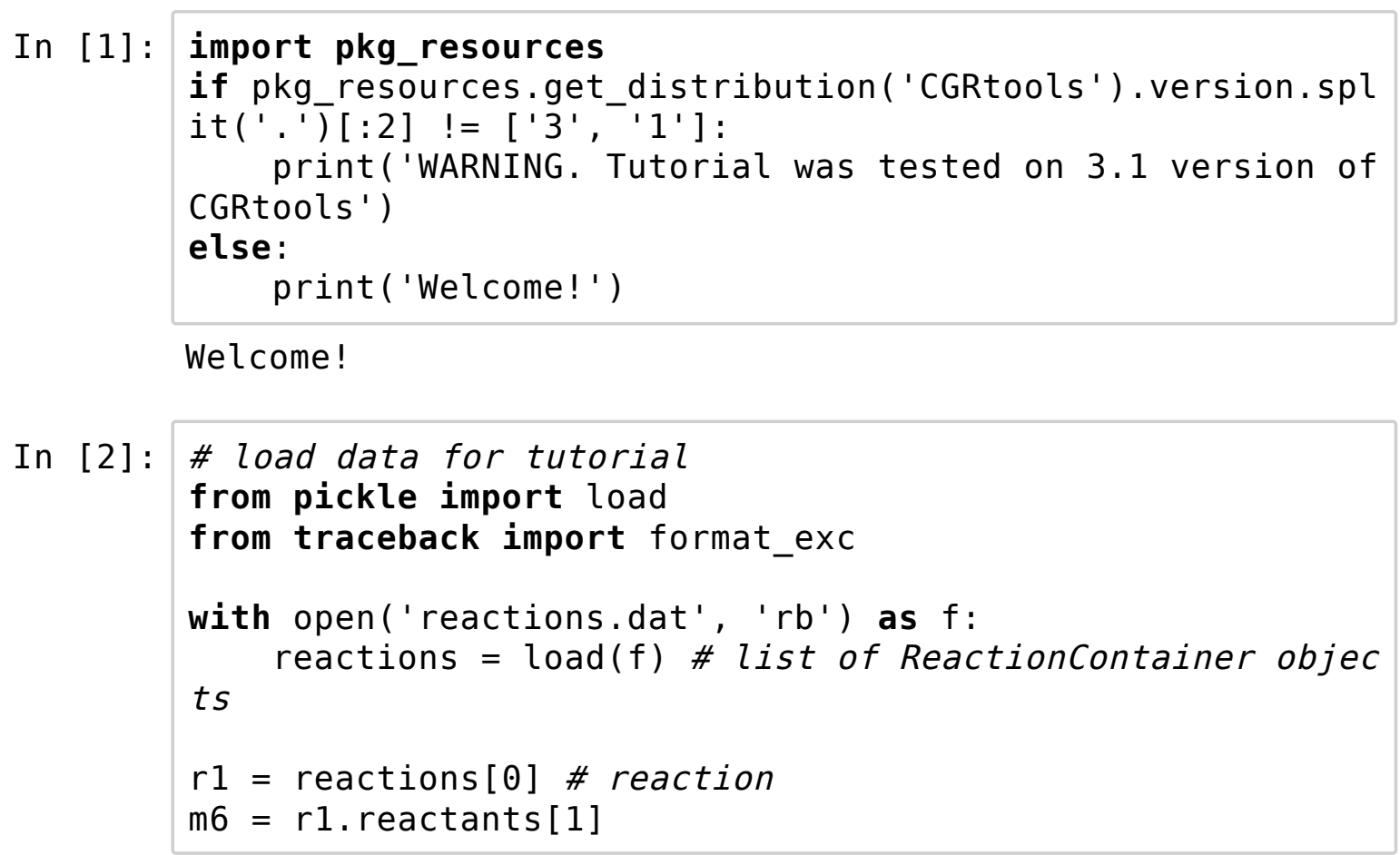

Reactor objects stores single transformation and can apply it to molecules or CGRs.

Transformations is ReactionContainer object which in reactant side consist of query for matching group and in product side patch for updating matched atoms and bonds

In [3]: from CGRtools import CGRreactor \# import of Reactor from CGRtools.containers import * \# import of required ob jects 


\subsection{Products generation}

Reactor works similar to ChemAxon Reactions enumeration.

Example here presents application of it to create esters from acids.

First we need to construct carboxy group matcher query. Then, ether group need to be specified.

Atom numbers in query and patch should be mapped to each other. The same atoms should have same numbers.

In [4]: acid = QueryContainer() \# this quer $y$ matches acids. Use construction possibilities.

acid.add_atom(\{'element': 'C', 'neighbors': 3\}) \# add $C$ arboxyl carbon. Hybridization is irrelevant here

acid.add_atom(\{'element': '0', 'neighbors': 1\}) \# add $h$ ydroxyl oxygen. Hybridization is irrelevant here acid.add_atom('0') \# add $c$ arbonyl oxygen. Number of neighbors is irrelevant here. acid.add_bond $(1,2,1)$ \# create single bond between carbo $n$ and hydroxyl oxygen

acid.add_bond $(1,3,2)$ \# create double bond print (acid)

$[C ; 3 ;](-[0 ; 1 ;])=[0]$

In [5]: methyl_ester = MoleculeContainer() \# create patch - how acrboxyl group should be changed. We write methylated gro up

methyl_ester.add_atom(' C', 1) \# second argument is predef ined atom mapping. Notice that mapping corresponds...

methyl_ester.add_atom('0', 2) \# ... to order in already $c$ reated acid group. Atom 2 is released water.

methyl_ester.add_atom ('0', 4)

methyl_ester.add_atom('0', 3)

methyl_ester.add_atom( 'C', 5)

methyl_ester.add_bond $(1,4,1)$

methyl_ester.add_bond $(1,3,2)$

methyl_ester.add_bond $(4,5,1)$

\# No bond between atom 1 and atom 2. This bond will be br oken.

methyl_ester.calculate2d()

methyl_ester

Out [5]:<smiles>COC=O</smiles> 
In [6]: m6.reset_query_marks() \# required for correct matching m6 \# acid

out [6] :<smiles>O=C(O)C(=O)O</smiles>

In [7]: template = ReactionContainer([acid], [methyl_ester]) \# me rge query and patch in template, which is ReactionContain er reactor $=$ CGRreactor(template) \#R eactor is initialized reacted acid $=$ reactor $(\mathrm{m} 6)$ \# a pplication of Reactor to molecule

In [8]: reacted_acid.calculate2d(scale=2) \# calculate coordinates reacted_acid \# desired methylated ester have been $g$ enerated

out [8] :<smiles>COC(=O)C(=O)O</smiles>

O

One can notice presence of separate oxygen (water) and ester group.

The second group can substituted by calling reactor on observed product.

In [9]: reacted_acid.reset_query_marks() \# this is new molecule a nd query marks need to be set

second_stage $=$ reactor $($ reacted_acid) \# apply transformati on on product of previous transformation second_stage.calculate2d(scale=2) \# recalculate coordina tes for correct drawing second_stage

out [9] :<smiles>COC(=O)C(=O)OC</smiles> 
second_stage has 3 components in a single MoleculeContainer object. We can split it into individual molecules and place all molecules into ReactionContainer object. Since in CGRtools atom-to-atom mapping corresponds to numbering of atoms in molecules, the resulting product has AAM according to the rule applied. Thus, reaction has correct AAM and nothing special should be made to keep or find it.

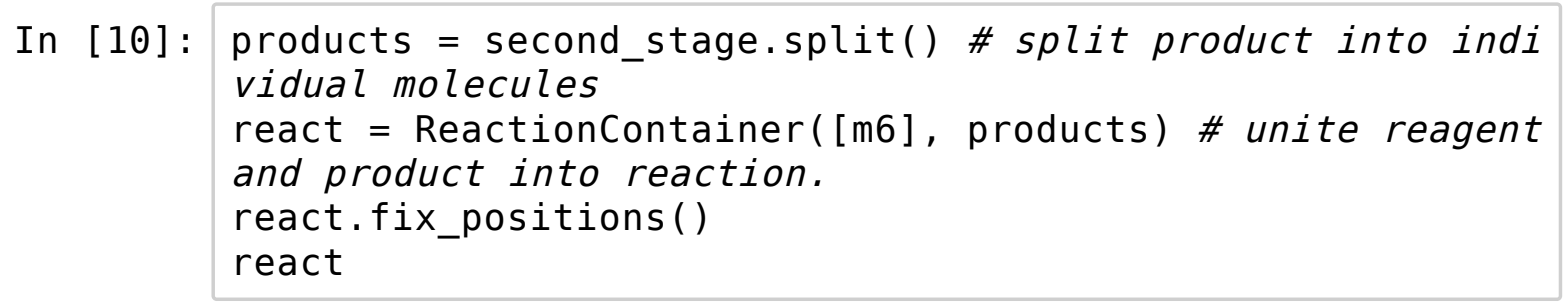

out $[10]$ :<smiles>O=C(O)C(=O)O</smiles><smiles>COC(=O)C(=O)OC</smiles>

For multicomponent reactions one can merge molecules of reactants into single MoleculeContainer object and apply reactor on it.

It is possible to generate all available products in case that molecule has several groups matching the query.

In [11]: m6copy = m6.copy() \# let's try to use molecule with sever al groups mathcing query

m6copy.atom(5). isotope $=13$ \# isotope mark is added to se $e$ the difference in products

m6copy.reset_query_marks() \# query marks need to be calcu lated

enums = set ()$\quad$ \# the set enums is used to sel ect structurally diverse products

for $\mathrm{m}$ in reactor(m6copy, limit $=0)$ : \# limit=0 is enumerati on of all possible products by reactor

print $(\mathrm{m}) \quad$ \# print signatures for observed molecules. Notice presence of water as component of product

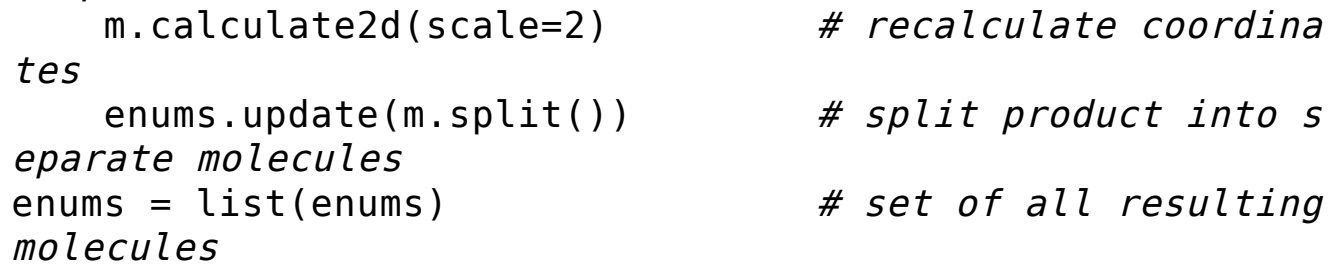


In [12]: m6copy

out [12] :<smiles>O=C(O)C(=O)O</smiles>

Let's have a look at molecules in set:

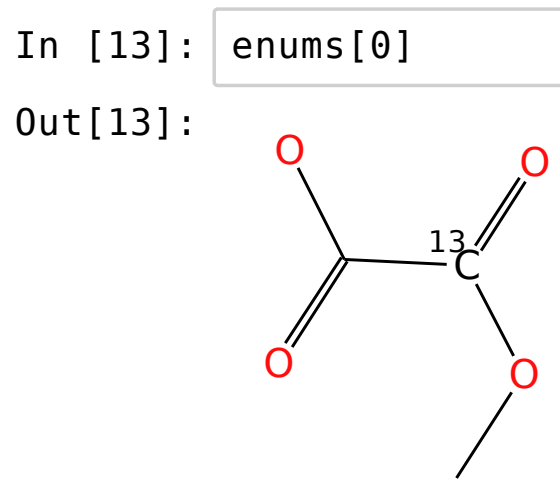

In [14]: enums [1]

out [14] :

$\mathrm{O}$

In [15]: enums [2]

Out [15] :

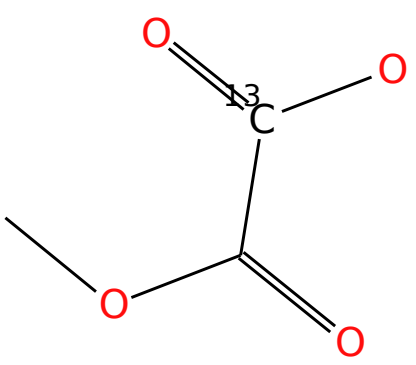

\subsection{MetaReactions (reactions on CGRs).}

Reactor could be applied to CGR to introduce changes into reaction.

\subsubsection{Example of atom-to-atom mapping fixing.}


In [16]: reactions[1] \# reaction under study

out [16] :

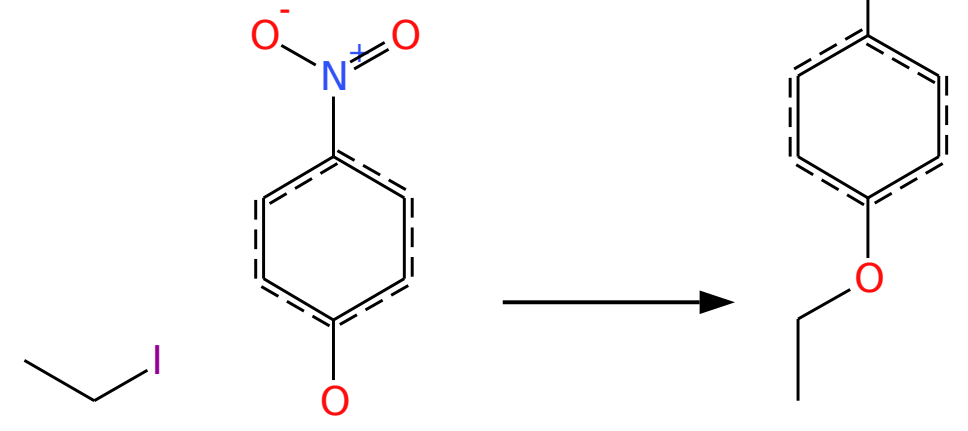

In [17]: $\operatorname{cgr}=\sim$ reactions[1] \# generate reaction CGR print (cgr)

$C-C(-I) .0-C: 1: C: C: C(: C: C: 1)-[N+](-[0-])=0>C-C(. I)-0-C: 1:$

$C: C: C(: C: C: 1)-[N+](=0)-[0-]$

In [18]: cgr.centers_list \# reaction has two reaction centers. [10 $, 11,12]$ - pseudo reaction appeared due to $A A M$ error

Out $[18]$ : $[[2,3,13],[10,11,12]]$

reaction has AAM error in nitro-group
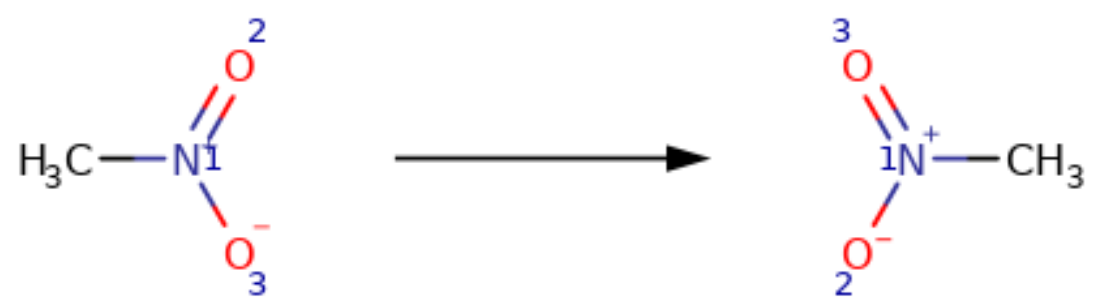

Lets try to use Reactor for AAM fixing 
In [19]: nitro = QueryCGRContainer() \# construct query for invalid reaction center - CGR of wrongly mapped nitro-group

nitro.add_atom(\{'element': 'N', 'charge': 1, 'p_charge':

1\}) \# atom 1

nitro.add_atom(\{'element': '0', 'charge': 0 , 'p_charge':

-1\}) \# atom 2. Notice that due to AAM error charge was ch anged

nitro.add_atom(\{'element': '0', 'charge': -1, 'p_charge':

$0\})$ \# atom 3. Notice that due to AAM error charge was cha nged

nitro.add_atom('C') \# atom 4

nitro.add_bond $(1,2,\{$ 'order': 2, 'p_order': 1\}) \# bond $b$ etween atoms 1 and 2. Due to AAM error bond is dynamic (' $2>1$ ' type)

nitro.add bond(1,3, \{'order': 1, 'p order': 2\}) \# bond $b$ etween atoms 1 and 3. Due to $A A M$ error bond is dynamic (" $1>2^{\prime}$ type)

nitro.add bond $(1,4,1)$ \# ordinary bond

print (nitro)

\# this query matches reaction center in CGR appeared due to $A A M$ error.

$[\mathrm{N}+](-[0-])(=[0])-[\mathrm{C}]>>[\mathrm{N}+](=[0])(-[0-])-[\mathrm{C}]$

In [20]: nitro < cgr \# query matches CGR of reaction with error.

out [20]: True

In [21]: valid_nitro = MoleculeContainer() \# construct nitro group without dynamic atoms. Notice that atom order should corr espond object nitro

valid_nitro.add_atom(\{'element': 'N', 'charge': 1\}) \# ord inary $N$ atom

valid_nitro.add_atom(\{'element': '0', 'charge': -1\}) \# or dinary negative $\bar{l} y$ charged oxygen atom

valid_nitro.add_atom('0')

dinary oxygen atom

valid_nitro.add_bond(1, 2, 1) \# ordinary single bond valid_nitro.add_bond $(1,3,2)$ \# ordinary double bond print (valid_nitro)

$[0-]-[N+]=0$

In [22]: valid_nitro.calculate2d()

valid_nitro \# this is correct representation of group.

Out [22] :

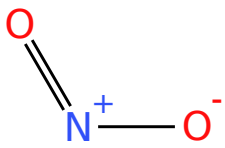


Now time to prepare and apply Template to CGR based on reaction with incorrect AAM.

Template is Reaction container with query in reactants and patch in products

In [23]: template = ReactionContainer([nitro], [valid_nitro]) \# te mplate shows how wrong part of CGR is transformed into co rrect one.

print(template) \# notice complex structure of query: CGR signature is given in braces, then $>$ and molecule signat ure

$\{[\mathrm{N}+](-[0-])(=[0])-[\mathrm{C}]>>[\mathrm{N}+](=[0])(-[0-])-[\mathrm{C}]\}>>[0-]-[\mathrm{N}+]$ $=0$

Reactor class accept single template. Existence of dynamic bond in it is not a problem.

In [24]: reactor $=$ CGRreactor (template)

Reactor object is callable and accept as argument molecule or CGR.

NOTE: fixed is new CGR object

In [25]: fixed $=$ reactor(cgr) \# fix CGR

CGRreactor returns None if template could not be applied, otherwise patched structure is returned.

In [26]: print(fixed)

$C-C(-I) .0-C: 1: C: C: C(: C: C: 1)-[N+](-[0-])=0>>-C(. I)-0-C: 1:$

$C: C: C(: C: C: 1)-[N+](-[0-])=0$

$C-C(-I) \cdot 0-C: 1: C: C: C(: C: C: 1)-[N+](-[0-])=0$

$C-C(. I)-0-C: 1: C: C: C(: C: C: 1)-[N+](-[0-])=0$

One can see that nitro group has no dynamic bonds any more. CGR corresponds only to substitution.

In [27]: fixed.centers list \# reaction center appeared due to AAM error before does not exist. Only 1 reaction center is fo und

out $[27]: \quad[[2,3,13]]$ 
Here is depiction of observed CGR (external software was used). Notice absence of wrong reaction center.<smiles>CC(C)Oc1ccc([N+](=O)[O-])cc1</smiles>

\subsubsection{Reaction transformation}

Example of E2 to SN2 transformation.

E2 and SN2 are concurrent reactions. We can easily change reaction center of E2 reaction to SN2. It could be achieved by substitution of reaction center corresponding to double bond formation in E2 reaction by the one corresponding to formation of new single bond with base as in SN2.

In [28]: from CGRtools import CGRreactor, CGRpreparer

from CGRtools.containers import QueryCGRContainer, Reacti onContainer

from CGRtools.files import MRVread, SDFwrite

from io import StringI0

In [29]: e2 = next (MRVread('e2.mrv')) \# read 22 reaction from Chem Axon MRV file

e2

Out [29] :
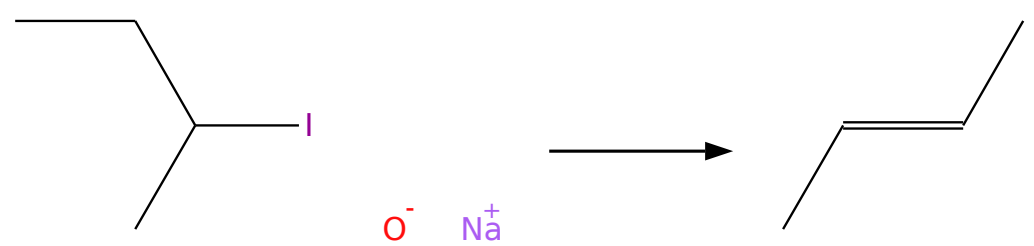

$\mathrm{O} \quad \mathrm{I}^{-} \mathrm{Na}^{+}$ 
In [30]: \# create CGR query for E2 reaction side

e2query = QueryCGRContainer()

e2query.add atom(' $\mathrm{C}$ ', 1) \# create carbon with mapping num ber 1

e2query.add_atom(' $\mathrm{C}$ ', 2) \# create carbon with mapping num ber 2

\# addition of any halogen atom

e2query.add_atom(\{'element': ['I', 'Cl', 'Br'], 'neighbor $\mathrm{S}^{\prime}:$ 1, 'p_neighbors': 0 , 'charge': 0 , 'p_charge': -1\}, 3) \# addition of $\mathrm{OH}-, \mathrm{RO}-, \mathrm{SH}-, \mathrm{RS}-$ groups

e2query.add_atom ( \{'element': ['0', 'S'], 'neighbors': [0,

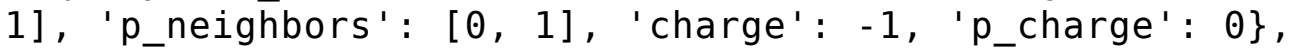
4)

e2query.add_bond $(1,2,\{$ 'order': 1, 'p_order': 2\}) \# bond between two carbon corresponds to formation of double fro $m$ single

e2query.add bond ( $1,3,\{$ 'order': 1, 'p_order': None $\})$ \# $b$ ond between carbon and halogen breaks in E2 reaction print(e2query) \# it is CGR of E2 reaction center

$[\mathrm{C}](-[\mathrm{C}])-[\mathrm{I}, \mathrm{Cl}, \mathrm{Br} ; 1 ;] .[0, \mathrm{~S} ;<01>;-]>>[\mathrm{C}](=[\mathrm{C}]) .[\mathrm{I}, \mathrm{Cl}, \mathrm{Br} ; 0$ ;- ]. $[0, \mathrm{~S} ;<01>$; ]

In [31]: e2_cgr $=\sim$ e2 \# compose reaction into CGR

e2_cgr.reset_query_marks() \# prepare to isomorphism

CGR is the following (depicted by external software)

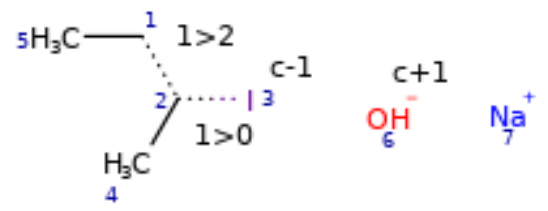

In [32]: e2query < e2_cgr \# E2 CGR pattern works!

out [32]: True 
In [33]: \# create patch creating SN2 reaction. Notice that orderin $g$ of atoms correspond to that of E2 CGR query sn2patch = QueryCGRContainer()

sn2patch.add_atom $(\{\}, 1)$ \# save atom unchanged. We don't specify atom type since it will be taken from E2 query sn2patch.add atom $(\{\}, 2)$ \# it is central atom. We don't $s$ pecify atom type since it will be taken from E2 query sn2patch.add_atom(\{'charge': 0 , 'p_charge': -1\}, 3) \# ele ments list with same order of elements [I, $\mathrm{Cl}, \mathrm{Br}]$ could be used as well

sn2patch.add_atom(\{'charge': -1, 'p_charge': 0$\}, 4)$

sn2patch.add_bond $\left(1,2,\left\{\operatorname{order}^{\prime}: 1\right.\right.$, 'p_order': 1\}) \# set carbon - carbon single bond that is unchanged in SN2 reac tion

sn2patch.add_bond( $1,3,\{$ 'order': 1, 'p_order': None $\}$ ) \# this bond is broken in SN2 reaction

sn2patch.add bond $(1,4,\{$ 'order': None, 'p_order': 1\}) \# it corresponds to formation of bond $O(S)-C$ bond in SN2 re action

In [34]: reactor $=$ CGRreactor(ReactionContainer([e2query], [sn2pat ch])) \# create template and pass it to Reactor $\mathrm{sn2}$ cgr $=$ reactor $(\mathrm{e} 2$ _cgr) \# apply Reactor on E2 reaction

In [35]: print(sn2_cgr)

$\mathrm{C}-\mathrm{C}(-\mathrm{C}-\mathrm{C})(.[0-])-\mathrm{I} .[\mathrm{Na}+]>>\mathrm{C}-\mathrm{C}(-\mathrm{C}-\mathrm{C})(-0) \cdot[\mathrm{I}-] \cdot[\mathrm{Na}+]$

It is depiction of CGR produced by Reactor (external software is used)<smiles>CC(C)C(C)(O[AlH2])[Te][Te]</smiles>

In [36]: \# decompose CGR into reaction

preparer = CGRpreparer( )

sn2 = preparer.decompose $\left(\mathrm{sn} 2 \_\mathrm{cg} r\right)$

In [37]: sn2.calculate2d()

sn2 \# reaction has the same reagents like $E 2$ above, but $p$ roducts correspond to SN2 reaction

Out [37] :
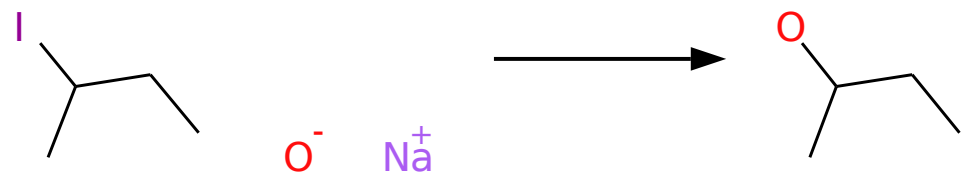


\section{Input-output operations}

(c) 2019, Dr. Ramil Nugmanov; Dr. Timur Madzhidov; Ravil Mukhametgaleev

Installation instructions of CGRtools package information and tutorial's files see on https://github.com/cimm-kzn/CGRtools

NOTE: Tutorial should be performed sequentially from the start. Random cell running will lead to unexpected results.

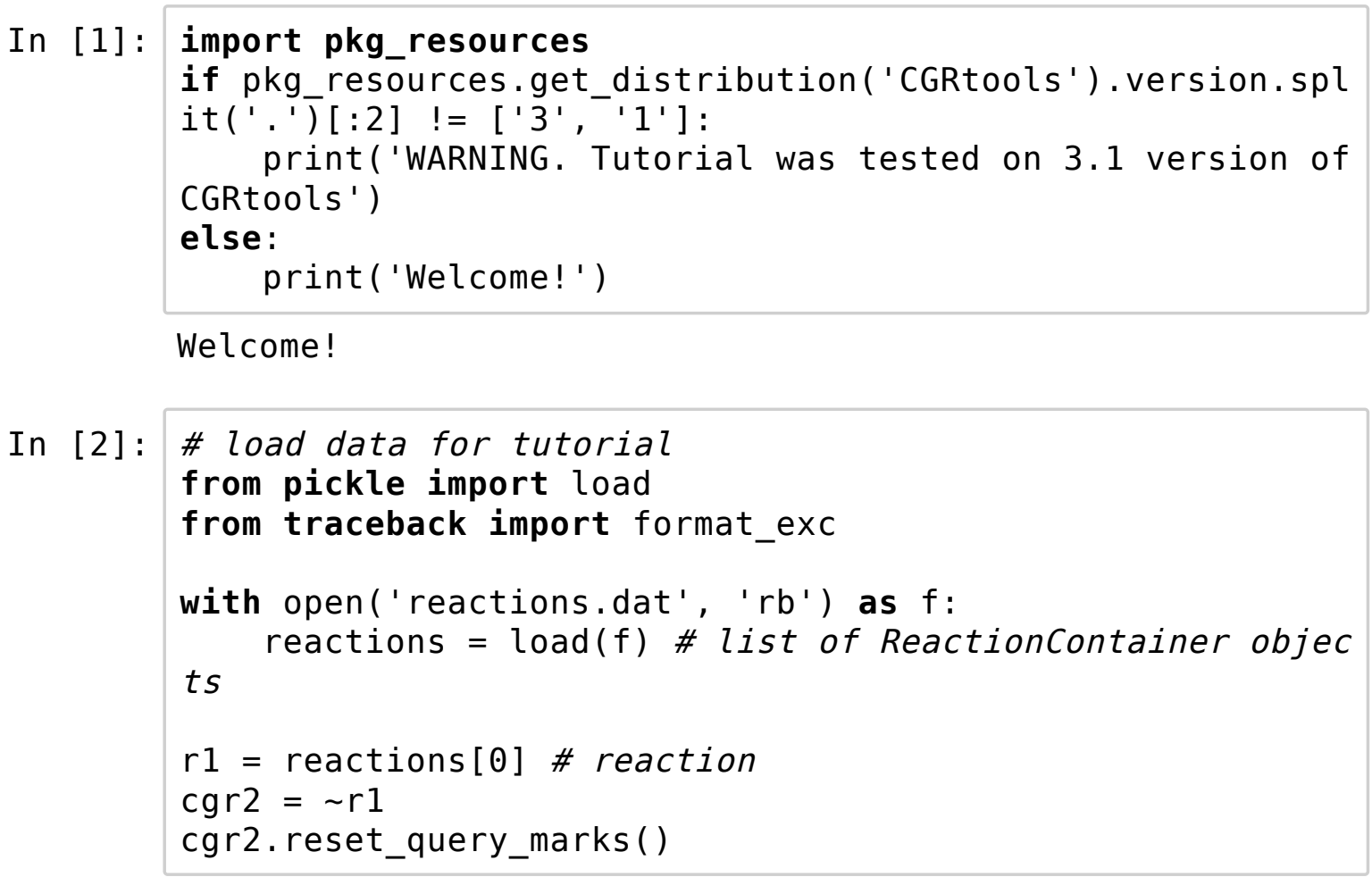

CGRtools.files subpackage contains file readers and writers classes.

\subsection{RDF reader}

RDFread class can be used for RDF files reading. Instance of this class is file-like object which support iteration, has a method read() for parsing all data and context manager.

\subsubsection{Read file from disk}


In [3]: from CGRtools.files import * \# import all available reade rs and writers

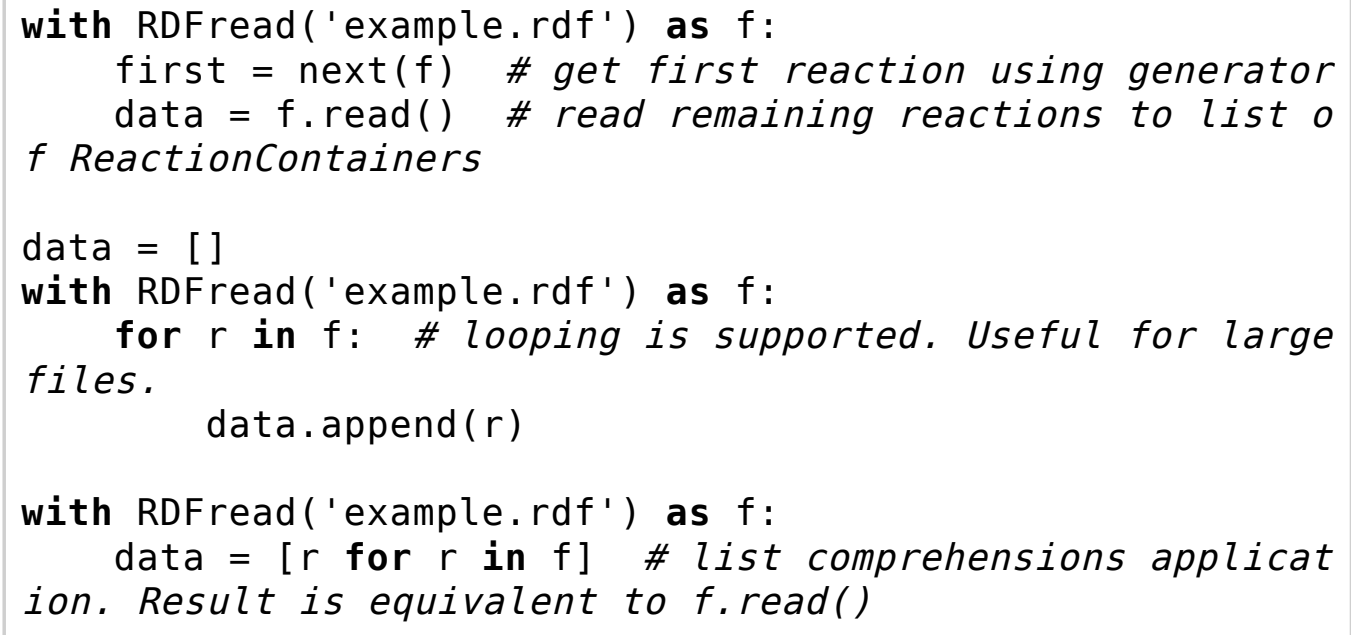

\section{OOP-stype Pathlib supported}

In [4]: from pathlib import Path

$$
\begin{aligned}
& \text { with RDFread(Path('example.rdf')) as } r \text { : \# OOP style call } \\
& r=\operatorname{next}(r)
\end{aligned}
$$

\section{opened files supported}

RDF file should be opened in text mode

In [5]: with open('example.rdf') as $f, \operatorname{RDFread}(f)$ as $r$ :

$r=\operatorname{next}(r)$ \# OOP style application

\subsubsection{Transparent loading from archives and network}

Readers designed transparently support any type of data sources.

Page https://cimm.kpfu.ru/seafile/f/aeaca685e3854ae2bbad/?dl=1 (https://cimm.kpfu.ru/seafile /f/aeaca685e3854ae2bbad/?dl=1) returns RDF file.

Data sources should be file-like objects. 
In [6]: from requests import get

from io import StringI0

\# get function return requested URL which has attribute $t$ ext.

\# in example this text is whole RDF stored in single stri $n g$.

\# RDFread does not support parsing of strings, but one ca

$n$ emulate files with data

\# instead of strings by using io.StringIo

with StringI0(get('https://cimm.kpfu.ru/seafile/f/aeaca68

5e3854ae2bbad/?dl=1'). text) as $f, \operatorname{RDFread}(f)$ as $r$ :

$r=\operatorname{next}(r)$

print( $r$, 'StringI0 downloaded from network data')

\# python support gzipped data. This example shows how to work with compressed

\# data directly without decompressing them to disk.

from gzip import open as gzip_open

with gzip_open('example.rdf.gz', 'rt') as $f, \operatorname{RDF} r e a d(f)$ a s $r$ :

$r=\operatorname{next}(r)$

print(r, 'gzipped file')

\# zip-files also supported out of the box

\# zipped files can be opened only in binary mode. io. Text Iowrapper can be used for transparent decoding them into text

from zipfile import ZipFile

from io import TextIOWrapper

with ZipFile('example.zip') as z, z.open('example.rdf') a s C:

with TextIOWrapper(c) as $f, \operatorname{RDFread}(f)$ as $r$ :

$r=\operatorname{next}(r)$

print $(r$, 'zip archive')

\# tar-file reading example

from tarfile import open as tar open

from io import TextIoWrapper

with tar open('example.tar.gz') as $t$ :

$c=\bar{t}$.extractfile ('example.rdf')

with TextIOWrapper(c) as $f, \operatorname{RDFread}(f)$ as $r$ :

$r=\operatorname{next}(r)$

print( $r$, 'gzipped tar archive')

$C-C-0 . C-C-0.0-C(=0)-C(-0)=0>>C-C-0-C(=0)-C(=0)-0-C-C$ Stri ngI0 downloaded from network data

$\mathrm{C}-\mathrm{C}-0 \cdot \mathrm{C}-\mathrm{C}-0 \cdot 0-\mathrm{C}(=0)-\mathrm{C}(-0)=0>>\mathrm{C}-\mathrm{C}-0-\mathrm{C}(=0)-\mathrm{C}(=0)-0-\mathrm{C}-\mathrm{C}$ gzip ped file

$C-C-0 \cdot C-C-0.0-C(=0)-C(-0)=0>>C-C-0-C(=0)-C(=0)-0-C-C$ zip archive

$C-C-0 \cdot C-C-0 \cdot 0-C(=0)-C(-0)=0>>C-C-0-C(=0)-C(=0)-0-C-C$ gzip

ped tar archive 


\subsection{Other Readers}

- SDFread - MOL, SDF files reader (versions v2000, v3000 are supported)

- MRVread - ChemAxon MRV files reader (Ixml parser is used)

- SMILESread - SMILES strings files reader (coho backend used). Every row should start with new SMILES

- INCHIread - INCHI strings files reader (INCHI trust backend used). Every row should start with new InChl

All files except MRV should be opened in text-mode

MRV requires binary mode open( '/path/to/data.mrv', ' rb')

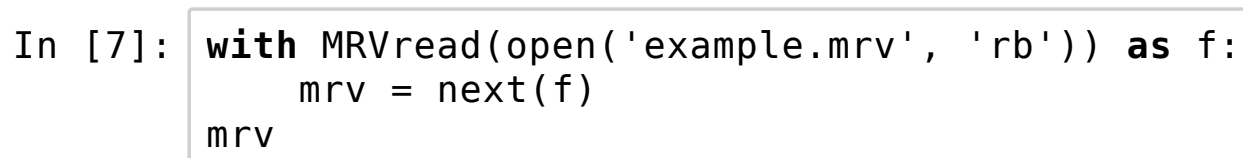<smiles>O=C([O-])C(=O)O</smiles>

\subsection{File writers}

Export in following file formats is supported:

- RDFwrite (v2000) - molecules and reactions export in RDF format

- SDFwrite (v2000) - molecules and CGR export in SDF format

- MRVwrite - molecules and reactions export in MRV format

Writers has the same API as readers. All writers work with text-files Writers has write method which accepts as argument single reaction, molecule or CGR object

In [8]: with RDFwrite('out.rdf') as $f:$ \# context manager supporte $d$

for $r$ in data:

f.write $(r)$

\# file out.rdf will be overriden 
In [9]: $f=$ RDFwrite('out.rdf') \# ongoing writing into a single $f$

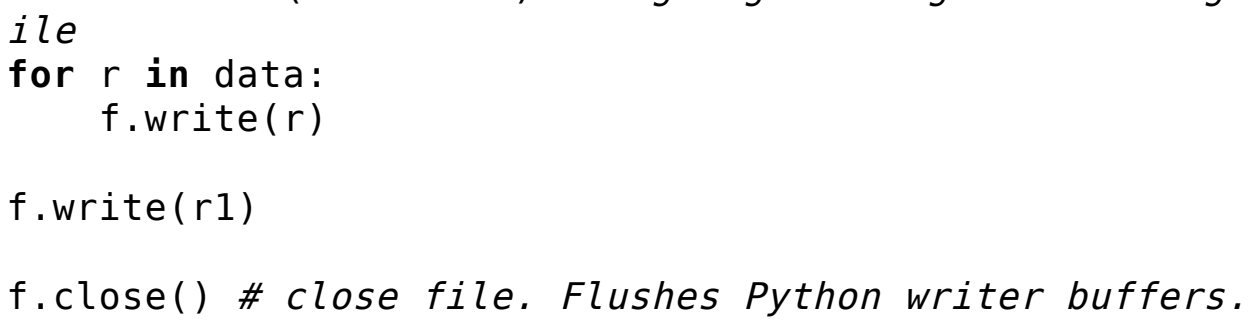

\subsection{CGR can be stored in MDL SDF and loaded from.}

White-paper with SDF-CGR specification is described in manusript Supporting Materials. 
In [10]: from CGRtools.files import *

from io import StringI0

with StringI0() as $f$, SDFwrite( $f)$ as $w$ :

w.write(cgr2) \# file writing in SDF format

$\mathrm{mdl}=\mathrm{f}$.getvalue() \# get formatted file to print out

print(mdl) \# It is how CGR looks like.

\# Notice that most of field are conventional MOL fields,

$S$-queries are used for dynamic bond and atom specificatio

$n$ 


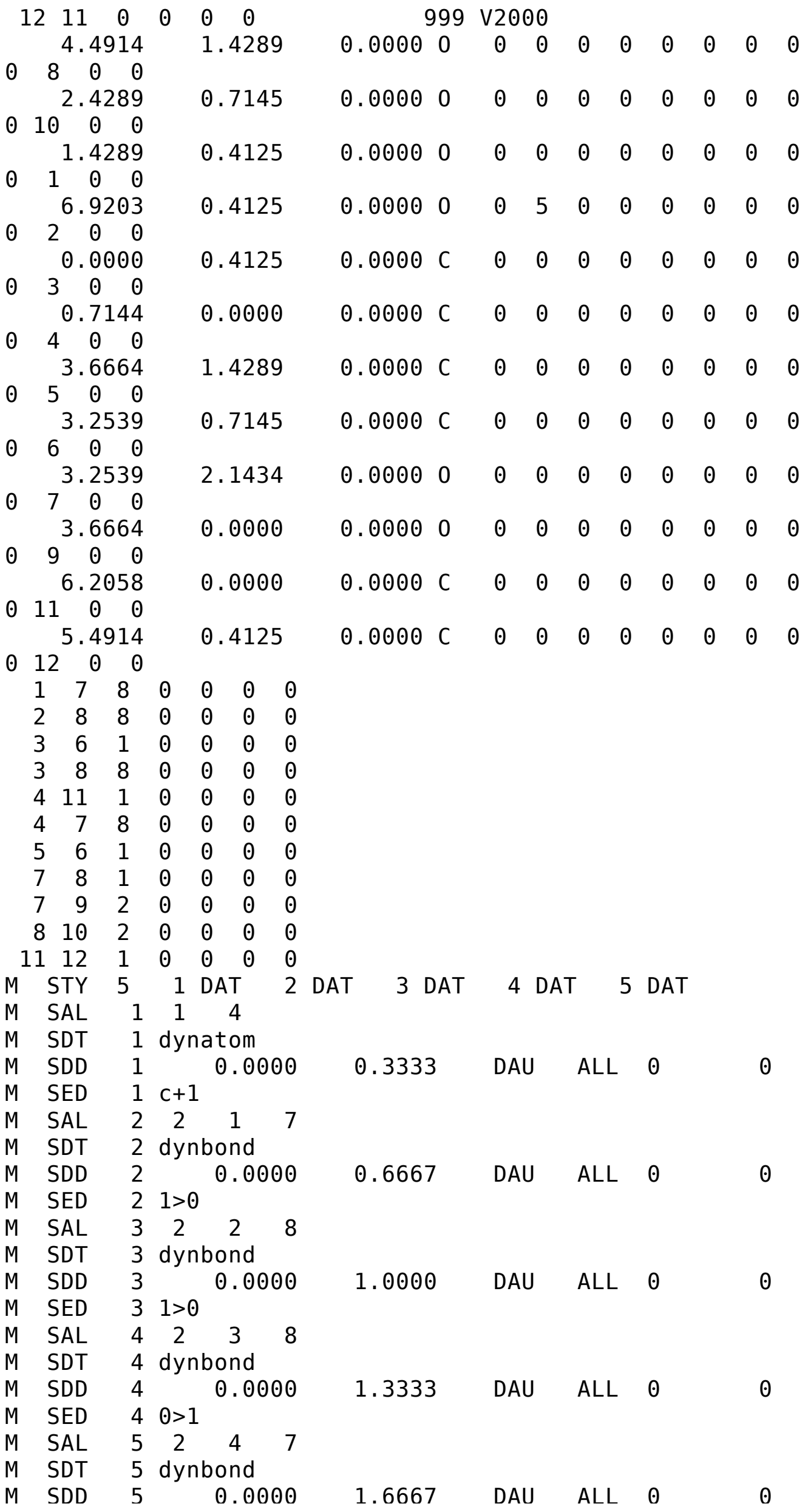




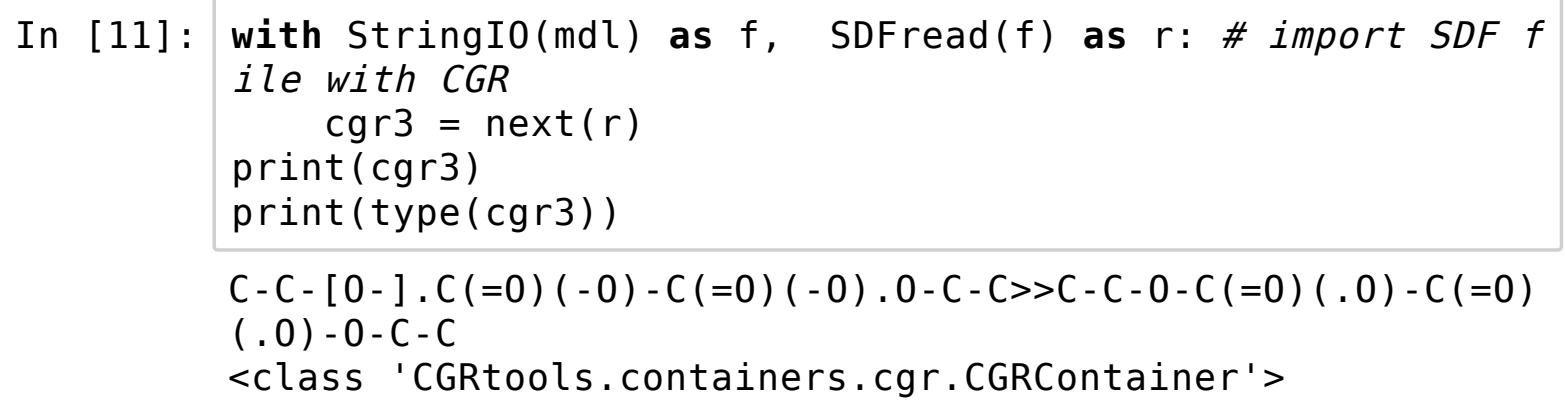

\subsection{Pickle support}

CGRtools containers fully support pickle dumping and loading.

Moreover backward compatability is declared since 3.0.

Any new version of library can load dumps created with older version.

Pickle dumps are more compact than MDL files and could be used as temporal storage.

In [12]: from pickle import loads, dumps

In [13]: loads(dumps( $r 1)$ ) \# load reaction from Pickle dump Out [13] :<smiles>CCOC(=O)C(=O)OCC</smiles> 